\title{
Monocrystalline methylammonium lead halide perovskite materials for photovoltaics
}

Anna Capitaine ${ }^{1}$, Dr. Beniamino Sciacca ${ }^{*}$

\footnotetext{
${ }^{1}$ CNRS- Aix-Marseille Université - CINaM, Campus de Luminy, 13288 Marseille, France

e-mail: beniamino.sciacca@univ-amu.fr, tel: +33660362822
} 


\section{Contents}

I Intro $\quad 3$

II Acronyms 4

III MAPX bulk crystals and thin films for solar cells $\quad 5$

1 Bulk Crystal Synthesis

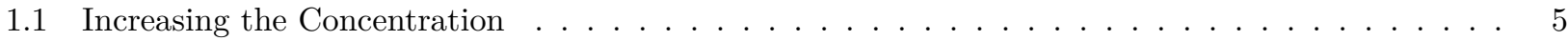

1.1.1 Solvent Evaporation $-\mathrm{SE} \ldots \ldots \ldots \ldots \ldots \ldots \ldots$

1.1.2 Liquid diffused separation induced crystallization - LDSC . . . . . . . . . . . . . . 5

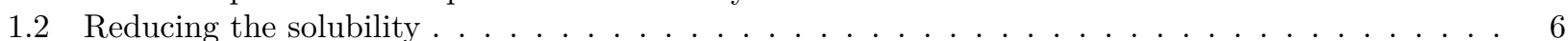

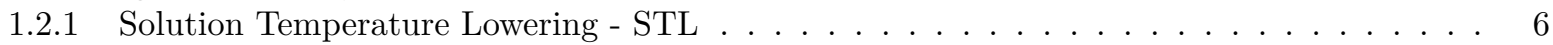

1.2.2 Antisolvent Vapor Crystallization - AVC . . . . . . . . . . . . . . . . . . 7

1.2.3 Inverse Temperature Crystallization - ITC $\ldots \ldots \ldots \ldots \ldots$

2 Single crystal thin films synthesis $\quad 10$

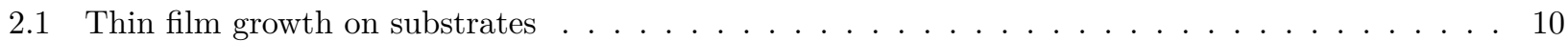

2.1.1 Space Limited Growth - SLG . . . . . . . . . . . . . . . . . . . . . 10

2.1.2 Cavitation triggered asymetrical crystallization - CTAC $(97) \ldots \ldots \ldots \ldots \ldots \ldots$

2.1 .3 Epitaxial Growth . . . . . . . . . . . . . . . . . . . . . . . . . 12

2.1.4 Conversion Methods . . . . . . . . . . . . . . . . . . . . . . . . 12

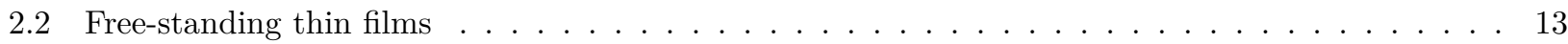

2.2 .1 Surface tension controlled ITC $(98) \ldots \ldots \ldots \ldots \ldots \ldots \ldots \ldots \ldots$

2.2 .2 From Bulk Crystals . . . . . . . . . . . . . . . . . . . . . 13

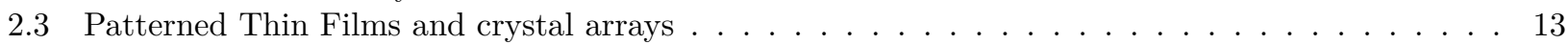

2.3 .1 Confined growth . . . . . . . . . . . . . . . . . . . . . 14

2.3 .2 Epitaxial Growth . . . . . . . . . . . . . . . . . . . . . . . 15

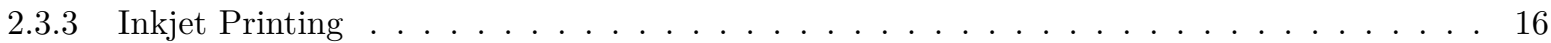

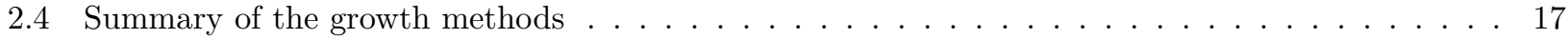

3 Optoelectronic properties $\quad 18$

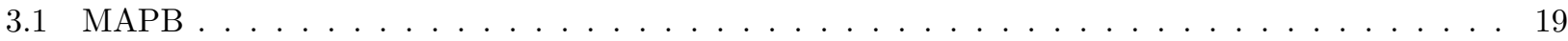

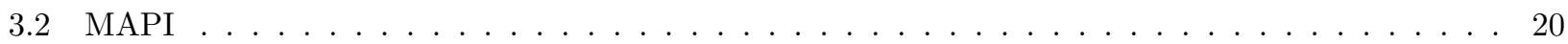

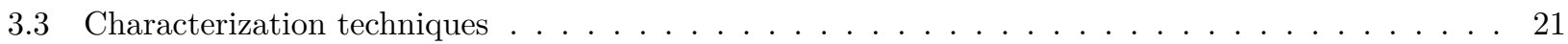

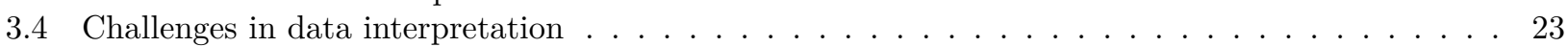

4 Single crystals stability $\quad \mathbf{2 4}$

4.1 Heat . . . . . . . . . . . . . . . . . . . . . . . . . . 25

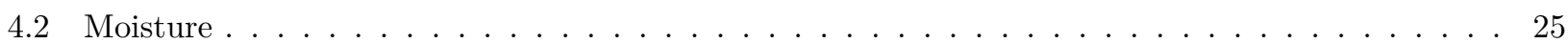

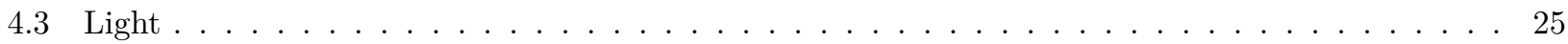

5 State of the art MAPX SC solar cell devices $\quad 26$

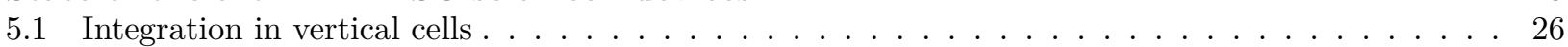

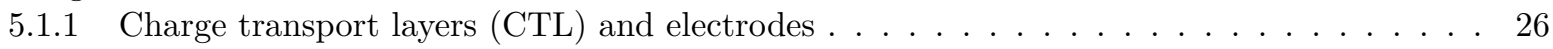

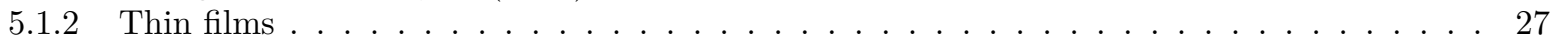

5.1 .3 Patterned thin films . . . . . . . . . . . . . . . . . . . 28

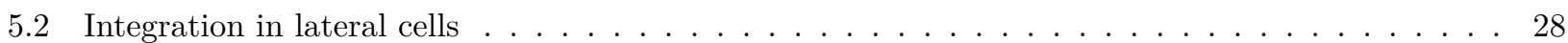

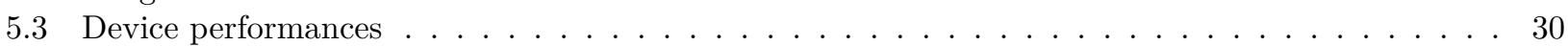

6 Why aren't single crystals overtaking polycrystalline solar cells ? 31

IV Potential and opportunities for SC beyond PC solar cells 33 
7 Anisoptropy in optoelectronic properties and performance

7.1 Anisotropy in MAPX . . . . . . . . . . . . . . . . . . . . . . 33

7.2 Impact of anisotropic charge transport on properties and performances . . . . . . . . . . . . . 34

7.3 Facets/termination dependent energy levels and charge transfer . . . . . . . . . . . . . . . 39

8 Perspectives and pathways for MAPX SC solar cells 40

8.1 Surface recombination . . . . . . . . . . . . . . . . . . . . . . . . 40

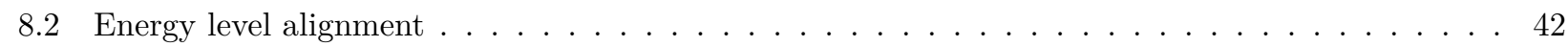

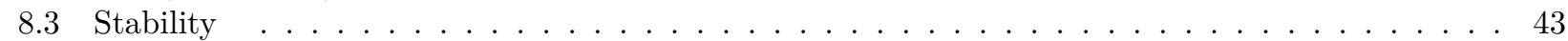

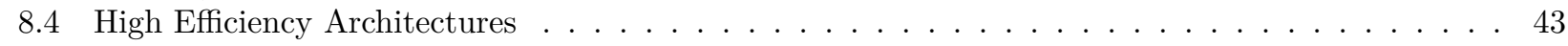

V Conclusion $\quad 44$ 


\section{Intro}

With a first paper in 2009 (1), lead halide perovskite solar cells have been gaining more and more interest, up to becoming the most promising material for future progress and developments in photovoltaic $(2,3)$. Thanks to high absorption coefficient, good optoelectronic properties and in particular long diffusion length, in only a decade, perovskite raised to a record power conversion efficiency (PCE) of $25.5 \%$ (4), approaching that of single crystalline silicon solar cells at lower cost. However, while for other materials such as GaAs, Si or Ge, the best solar cells were achieved with single crystals (SC), for perovskite the best cells have been so far achieved with polycrystalline (PC) thin films, despite the optoelectronic properties of perovskite SC are undoubtedly superior.

$\mathrm{SC}$ perovskite, free of grain boundaries, have traps density in the order of $10^{9}-10^{10} \mathrm{~cm}^{-3}, 6-7$ orders of magnitude lower than PC perovskite and comparable to silicon SC. Diffusion length exceeding $175 \mu \mathrm{m}(5)-$ the longest among common direct bandgap semiconductor (6) - and carrier mobility larger than $150 \mathrm{~cm}^{2} \mathrm{~V}-1 \mathrm{~s}^{-1}(7)$ were reported for methylammonium lead iodide (MAPI) SC. Grain boundaries typically promote non-radiative recombination due to a high concentration of defects such as dangling bonds, impurities or vacancies, acting as traps for holes or electrons. In addition to having a detrimental impact on the optoelectronic properties of PC perovskites (8-12), grain boundaries were reported to act as initiation sites for device degradation by (i) providing faster pathways for ion migration (13-15), (ii) causing the introduction of defects under bias or under light exposure (16), (iii) facilitating water permeation (17-19) and (iv) inducing thermal instability (20-22). Although the record performance of PC perovskite solar cells is partly related to good defect tolerance (due to the bandgap being essentially free from deep level defects $(23))$, stability issues $(18,24,25)$ strongly hamper commercialization.

With superior optoelectronic properties and better stability, it would be expected for SC to boast high performance solar cells. PC-cells however, have received much more attention than SC solar cells, mainly do to easier and quicker fabrication of PC perovskite. While the number of publications for PC solar cells is in the order of one to two thousands of papers per year since approximately 2015, the first report of SC solar cell was made in 2015, and since then less than 20 papers were published on the subject (26). The highest PCE reported to date for a SC-perovskite solar cell is $21.9 \%$ (27), smaller than that of PC-solar cells built out of more complex materials (often mixed-halide and mixed-cation perovskite), and shows that SC perovskite can be grown in the form of thin film and integrated successfully in solar cells. However, it still does not give credit to the SC outstanding properties.

By taking methylamonnium lead halide perovskite (MAPX) SC as an example we aim at giving a full picture of the interest of SC lead halide perovkite for photovoltaic applications. MAPI in particular is highly interesting for solar cell application, with a bandgap around $1.6 \mathrm{eV}$, close to the optimal (28), and high optical absorption coefficient $\alpha=10^{4}-10^{5} \mathrm{~cm}^{-1}$ above $1.7 \mathrm{eV}(29)$. Recent progress in MAPB and MAPC SC growth methods has been made for applications such as photodetectors or image sensors and there is a growing interest for solar cells.

Recent reviews in the literature comprehensively describe growth methods, properties and integration of MAPX SC in optoelectronic devices $(17,25,30-43)$. The originality of the present review is to take an holistic approach to (i) critically assess the potential of SC for solar cells; (ii) put in perspective growth methods, optoelectronic properties and device performance to give insights on why 5 years after the first report, SCsolar cells struggle to outperform PC-solar cells despite the outstanding material properties; (iii) regard the anisotropy in physico-chemical properties as a unique asset for perovskite SC to outperform PC. We review the different techniques developed to grow bulk crystals, thin films and patterned thin films in solution, highlighting recent progress. Next, we put in comparison the material properties of bulk crystal and thin films, illustrating the challenges for characterization and data interpretation, that have often lead to errors in the measurements of key properties such as the bandgap. Next, we focus on the strategies developed to integrate them in solar cells, and we identify the major hurdles that still need to be overcome for substantial breakthrough. We identify surface trap density as being one of the main issue limiting solar cells performance, in addition to the difficulty to grow very thin films of MAPX SC for efficient integration in devices. We propose surface design strategies to mitigate the impact of surface traps. Last, we focus on new perspectives of perovskite SC to outperform PC by taking advantage of anisotropy. Tuning crystallographic orientation during SC growth and chemical termination might be key for better charge extraction, stability and controlled charge migration, as opposed to $\mathrm{PC}$ perovskites that present a variety of grain orientation and surface termination. 
II Acronyms

\begin{tabular}{|c|c|}
\hline & Acronyms \\
\hline $1 \mathrm{P} / 2 \mathrm{P}$ & One/two photon (excitation) \\
\hline AAO & Anodized Aluminum Oxide \\
\hline $\mathrm{ACN}$ & Acetonitrile \\
\hline $\mathrm{AVC}$ & Antisolvent Vapor Crystallization \\
\hline BA & Benzylamine \\
\hline $\mathrm{BCP}$ & C60-Bathocuproine \\
\hline BL & Blocking Layer \\
\hline BSSG & Bottom-Seeded Solution Growth \\
\hline cAFM & Conductive Atomic Force Microscope \\
\hline $\mathrm{C}_{B}$ & Conduction Band \\
\hline CMTP & Continuous-Mass Transport Process \\
\hline CTAC & Cavitation Triggered Asymetrical Crystallization \\
\hline CTL & Charge Transport Layer \\
\hline DCM & Dichloromethane \\
\hline DMF & N,N-Dimethylformamide \\
\hline DTAB & Dodecyltrimethylammonium Bromide \\
\hline DMSO & Dimethyl Sulfoxide \\
\hline EQE & External Quantum Efficiency \\
\hline ETL & Electron Transport Layer \\
\hline FA & Formammonium \\
\hline $\mathrm{FF}$ & Fill Factor \\
\hline FTO & Fluorine doped Tin Oxide \\
\hline GBL & Gamma-Butyrolactone \\
\hline $\mathrm{HBr}$ & Hydrobromide \\
\hline HT & High-Temperature \\
\hline HTL & Hole Transporting Layer \\
\hline $\mathrm{HX}$ & Hydroalic solution \\
\hline ITC & Inverse Temperature Crystallization \\
\hline IS & Impedance Spectroscopy \\
\hline ITO & Indium Tin Oxide \\
\hline $\mathrm{J}_{S C}$ & Short-Circuit current \\
\hline LDSC & Liquid Diffused Separation Induced Crystallization \\
\hline LT & Low Temperature \\
\hline MA & Methylammonium \\
\hline $\mathrm{MACl}$ & Methylammonium Chloride \\
\hline MAPB & Methylammonium Lead Bromide Perovskite \\
\hline MAPC & Methylammonium Lead Chloride Perovskite \\
\hline MAPI & Methylammonium Lead Iodide Perovskite \\
\hline MAPX & Methylamonnium Lead Halide Perovskite \\
\hline $\mathrm{MCC}$ & Microchannel Confined Crystallization \\
\hline MSM & Metal-semiconductor-metal \\
\hline $\mathrm{OAB}$ & Oleylammonium Bromide \\
\hline ORAP & Ostwald Ripening Assisted Crystallization \\
\hline $\mathrm{PbX}_{2}$ & Lead Halide \\
\hline $\mathrm{PC}$ & Polycristalline \\
\hline PCE & Power Conversion Efficiency \\
\hline PDMS & Polydimethylsiloxane \\
\hline PD & Photodetector \\
\hline PEAI & Phenethylammonium Iodide \\
\hline PET & Polyethylene terephthalate \\
\hline PL & Photoluminescence \\
\hline PLQY & Photoluminescence Quantum Yield \\
\hline PTAA & Poly(triaryl amine) \\
\hline
\end{tabular}




\begin{tabular}{cc}
\hline & Acronyms \\
\hline RT & Room Temperature \\
SC & Single Crystal \\
SCLC & Space Charge Limited Current \\
SE & Solution Evaporation \\
SLG & Space Limited Growth \\
SRV & Surface Recombination Velocity \\
STITC & Surface tension controlled ITC \\
STL & Solution Temperature Lowering \\
TA & Transient Absorption \\
TCE & Trichloroethane \\
TFL & Trap-Filled Limit \\
ToF & Time-of-flight \\
TPV & Transient photovoltage \\
TRPL & Time-Resolved Photoluminescence \\
TSSG & Top-Seeded Solution Growth \\
V & Valence Band \\
$V_{O C}$ & Open-Circuit voltage \\
\hline
\end{tabular}

\section{MAPX bulk crystals and thin films for so- lar cells}

\section{Bulk Crystal Synthesis}

Crystallization methods of MAPX perovskite in solution aim at reaching the point of supersaturation of perovskite precursors by either raising their concentrations or reducing their solubility.

\subsection{Increasing the Concentration}

\subsubsection{Solvent Evaporation - SE}

In order to reach supersaturation of precursors in solution, the easiest is to gradually evaporate the growth solution. This solution is typically composed of the two precursors methylammonium (MA) and lead halide $\left(\mathrm{PbX}_{2}\right)$, whose concentration increases upon evaporation of the solvent (44). Both hydrohalic solutions (HX) and $\mathrm{N}, \mathrm{N}$-Dimethylformamide (DMF) proved to be suitable solvents to grow bulk crystals of MAPB; interesingly, $\mathrm{HBr}$ based solutions yield $\{110\}$ and $\{100\}$ faceted crystals $(44,45)$, whereas those grown in DMF exhibit larger $\{100\}$ facets (45) as showed in Figure 1a and b, suggesting that morphology control could be achieved by a rational choice of solvent. The addition of surfactants enabled for crystal facet engineering, via selfassembly. Single plates terminated by $\{001\}$ facets or single rods by $\{110\}$ facets, were obtained by using dodecyltrimethylammonium bromide (DTAB) or oleylammonium bromide (OAB) as surfactant, respectively. Interestingly, photoluminescence (PL) lifetime was found to be more than three times longer for single plates compared to the rods, which was interpreted by the authors as originating from anisotropic charge transport in the perovskite. However, crystals grown by this technique are typically small, with $80 \mu \mathrm{m}$ sides for the single plates and $20 \mu \mathrm{m}$ in width for the rods (46). Several days are needed to grow millimeter sized crystals with this technique (45).

\subsubsection{Liquid diffused separation induced crystallization - LDSC}

In this technique, the precursor concentration is increased by letting the solvent diffusing out into a denser liquid. Yao et al. were able to obtain high quality MAPB, MAPC and MAPI SC at room temperature, by adding silicone oil on top of a DMF-DMSO (dimethyl sulfoxide)-ACN (acetonitrile)-GBL (gamma-Butyrolactone) solution containing the dissolved MAPX perovskite precursors $\left(\mathrm{MAPb}, \mathrm{PbX}_{2}\right)$. Due to the lower density, the solution diffused towards the top, while the perovskite precursors, having a higher density, remained at the bottom, leading to a concentration increase as illustrated in Figure 1c. For MAPB SC the trap density was reported to be as low as $4.4 \cdot 10^{9} \mathrm{~cm}^{-3}$, the lifetime of $1 \mu \mathrm{s}$ and the optical bandgap of $2.15 \mathrm{eV}$. Several days are needed to achieve centimeter sized crystals (Figure 1d) (47). 
a

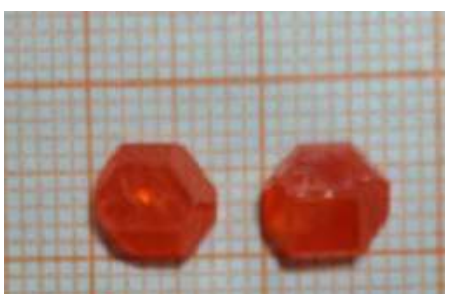

c

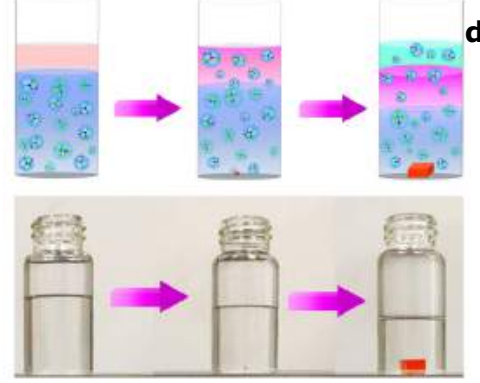

b

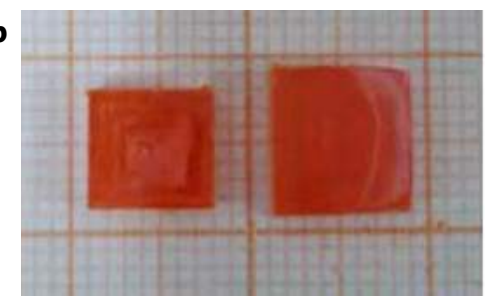

d

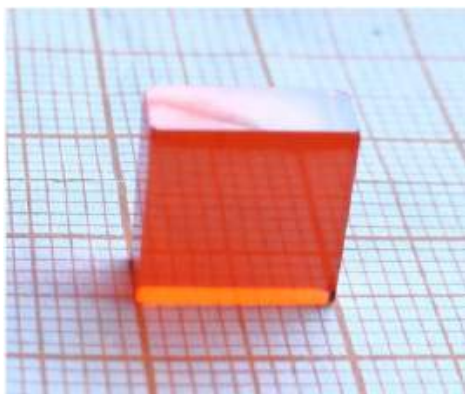

Figure 1 - Synthesis by raising concentration. Photographs of MAPB SC grown by solution evaporation in a) $\mathrm{HBr}$ b) DMF (from ref.(45)) c) Schematic illustration of SC-growth by the LDSC method d) Photograph of MAPB-SC grown using the LDSC method (from ref.(47)).

\subsection{Reducing the solubility}

Solubility can be reduced by either i) lowering the temperature (Solution Temperature Lowering - STL), ii) increasing the temperature (Inverse Temperature Crystallization - ITC) or iii) using an antisolvent (Antisolvent Vapor Crystallization - AVC). All of those techniques typically allow to grow bulk single crystals of a few cubic millimiters.

\subsubsection{Solution Temperature Lowering - STL}

Solubility being highly dependent on temperature, it is possible to trigger nucleation by either raising or lowering the temperature of a perovskite precursor solution. STL techniques, as the name indicates, consist in lowering the temperature in order to reach supersaturation, and are therefore applied to solutions in which precursors are less soluble at low temperatures than at high temperatures. The precursors typically used are MAX and lead acetate $\left(\mathrm{Pb}\left(\mathrm{CH}_{3} \mathrm{COOH}\right)_{2} \cdot 3 \mathrm{H}_{2} \mathrm{O}\right)$ in $\mathrm{HX}$.

First used by Poglitsh and Weber in 1987 (48), this general method is now largely used to grow high quality SC $(5,49-51)$. Dang et al. synthesized MAPI millimeter-size crystals $\left(10 \times 10 \times 8 \mathrm{~mm}^{3}\right)$ capped by (100) and (112) facets by gradually lowering the temperature of an HI-based precursor solution from $65^{\circ} \mathrm{C}$ to $40^{\circ} \mathrm{C}$ over the course of a month (49) (Figure 2a). Seeded methods can be used to speed-up the synthesis. First, a seed crystal is prepared, typically by the Poglitsh and Weber method (5, 50), and is thereafter placed either at the top or bottom of the solution, to have bottom-seeded solution growth (BSSG) or top-seeded solution growth (TSSG). Centimeter-size SC capped by (112) and (100) facets were obtained by slowly lowering the solution temperature from $80^{\circ} \mathrm{C}$ to $60^{\circ} \mathrm{C}$ at a rate of $5^{\circ} \mathrm{C}$ per hour (50), in a BSSG configuration (Figure 2b). The addition of chlorine $(\mathrm{MACl})$ to the precursor solution enabled to further speed up the crystallization process from weeks to days and to improve the quality of the MAPI SC (trap densities as low as $3.8 \pm 1.1 \cdot 10^{9} \mathrm{~cm}^{-3}$ ) (51).

Another approach to prepare MAPI seeds is to induce a thermal gradient in the solution. Precursors are gradually dissolved in the bottom of the solution which is hotter, enabling nucleation on a substrate fixed at the top of the solution (colder) and formation of a single seed, that can be grown in a bulk crystal in the TSSG configuration. This enabled the formation of millimiter sized bulk MAPI SC with impressive optoelectronic properties, such as a diffusion length of $175 \mu \mathrm{m}$ and lifetime approaching $100 \mu \mathrm{s}$, within days. The bandgap was reported to be $1.48 \mathrm{eV}(5)$. This is slightly smaller than MAPI PC and is due to the presence of an indirect bandgap at smaller energy, that is often not detected in thin PC film due to its low absorption coefficient (52). This will be discussed in more details in paragraph 8.4 .

However, in addition to STL being relatively long, it can induce the nucleation of defects, due to the high growth temperature $\left(\sim 100^{\circ} \mathrm{C}\right.$ is typical) $(53-55)$ or due to the phase transition from cubic-MAPI to tetragonalMAPI during cooling (53). 

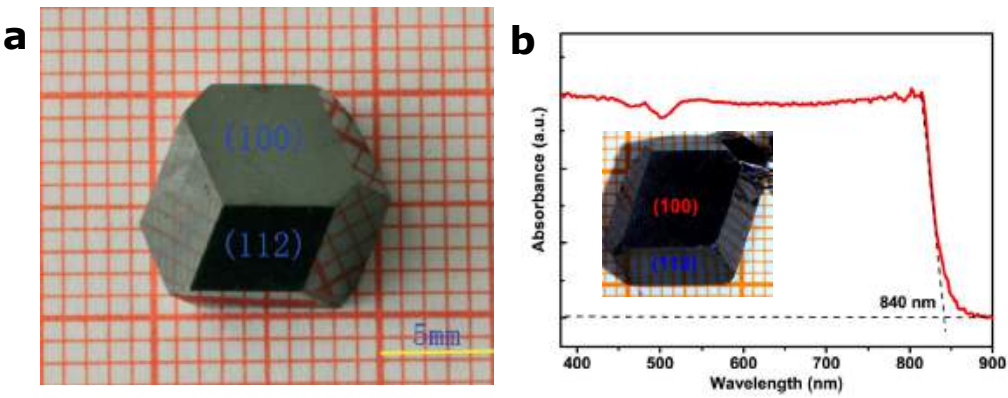

Figure 2 - MAPX-SC growth by STL. a) Image of MAPI exhibiting its natural facets (100) and (112) (from ref.(49)) b) UV-visible absorption spectrum for a (100) MAPI SC plate cut from a bulk SC and photograph of the MAPI bulk SC grown through the BSSG method (from ref.(50)).

\subsubsection{Antisolvent Vapor Crystallization - AVC}

The perovskite precursors MAX and $\mathrm{PbX}_{2}$ are typically dissolved in a solvent such as DMF or GBL. Nucleation is triggered by gradual evaporation and diffusion in the growth solution of an anti-solvent in which the precursors are not soluble (56-61), such as dichloromethane (DCM), diethylether, toluene, ethanol or ACN (62). A typical AVC setup is depicted in Figure 3d.

Bulk crystals of MAPI and MAPB with volume $>100 \mathrm{~mm}^{3}$ and trap densities of $3.3 \cdot 10^{10} \mathrm{~cm}^{-3}$ can be obtained within a week using DCM as an anti-solvent. Optical bandgaps of $2.21 \mathrm{eV}$ (MAPB) and $1.51 \mathrm{eV}$ (MAPI) were reported (Figure $3 \mathrm{a}$ and b) (56).

The morphology and the properties of the crystals are strongly influenced by the growth kinetics, that can be tuned by the solution concentration or by precise control of the anti-solvent diffusion. This was key to reduce pitting on the (100) facet (Figure 3c) (57) and obtain MAPB SC stable up to $300^{\circ} \mathrm{C}$ and with trap densities in the order of $10^{10} \mathrm{~cm}^{-3}(62)$. Control over the degree of supersaturation can be achieved by tuning temperature and flow rate of the anti-solvent while a seed crystal is fixed on a platform that rotates during crystal growth (Figure 3e). The rotation allows for a thinner growth boundary layer, homogeneous supersaturation, while hampering unwanted adsorption (63). Overall, faster growth and higher quality can be achieved.

AVC has the advantage to synthesize SC at room temperature, but in some case optoelectronic properties worsen due to anti-solvent contamination (53). 

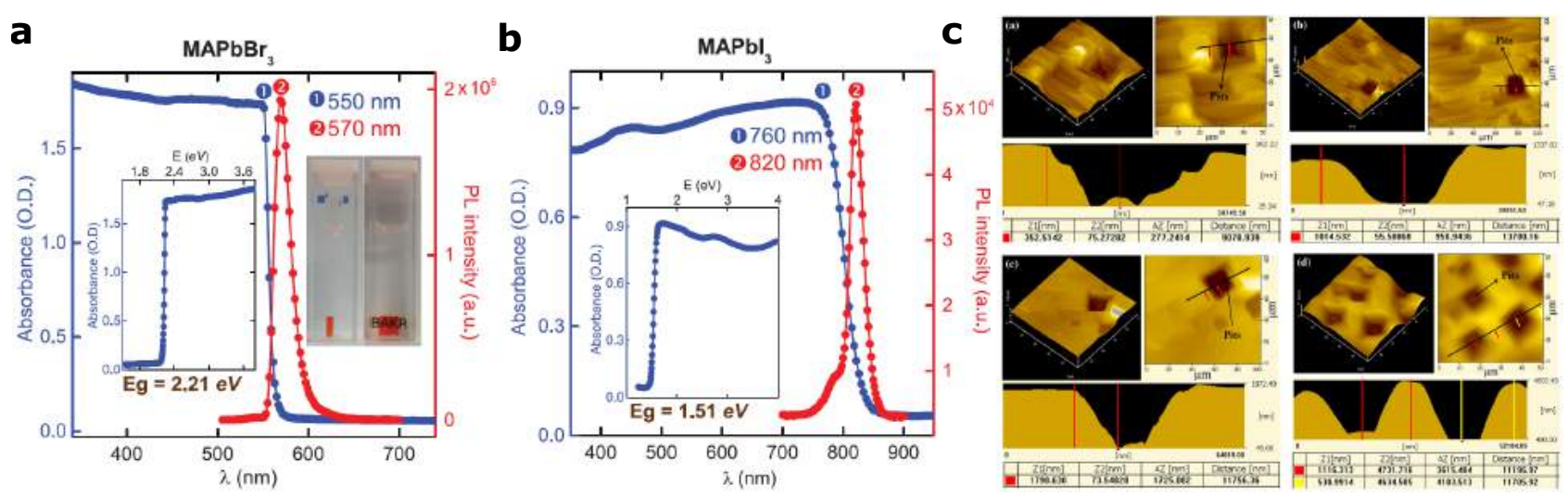

d

e
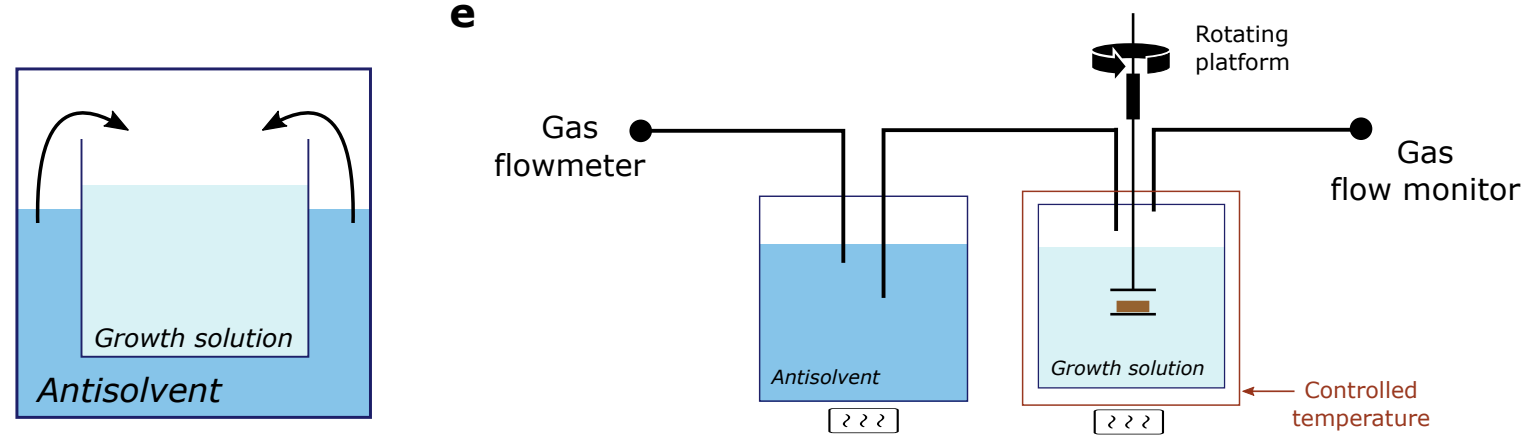

Figure 3 - MAPX-SC growth by AVC. MAPB (a) and MAPI (b) steady-state absorbance and photoluminescence (from ref.(56)); (c) AFM images of (100) surface of MAPB SC grown with different precursor concentrations: a 0.7 M; b $0.8 \mathrm{M}$; c $0.9 \mathrm{M}$; and d $1 \mathrm{M}$ (from ref.(57)); (d) Schematic illustration of a typical AVC set-up; (e) Optimized set-up developed by Zhang et al. (d and e from ref.(63)).

\subsubsection{Inverse Temperature Crystallization - ITC}

In ITC supersaturation is obtained by increasing the temperature, and is therefore applied to precursors whose solubility in a given solvent decreases with increasing temperature (exothermic dissolution). Typically the precursors (MAX and $\mathrm{PbX}_{2}$ ) are dissolved in GBA or GBL to form MAPI $(6,64-68)$, DMF to form MAPB $(66,67,69,70)$ or DMSO to form $\operatorname{MAPC}(66,71)$.

Millimeter sized crystals can be grown in a few hours as opposed to days, making this method much faster than the methods presented above (69). However, it was recently reported that unseeded ITC suffered from low yield as well as low reproducibility with over half of as obtained crystals being in fact polycrystalline (72).

Seeded ITC enables the synthesis of bulk SCs as large as many centimeters $(6,69)$, while the obtained SC can be used as seed multiple times by placing the growing crystal in a fresh precursor solution (66) (Figure 4a). The careful choice of crystallization temperature is key to obtain high material quality. MAPB SC crystals obtained by slowly heating a precursor solution from $25^{\circ} \mathrm{C}$ to $60^{\circ} \mathrm{C}$ over 20 days showed 3x better mobility and 10x lower trap densities compared to MAPB-SC grown at $100^{\circ} \mathrm{C}$ (Figure $4 \mathrm{~b}$ and c) (70).

Comparison between properties of MAPB SC grown at RT by LDSC (see section 1.1.2) and ITC (at $90^{\circ} \mathrm{C}$ ) supports the hypothesis that high-temperature growth is detrimental for crystal quality: LDSC grown sample exhibited 10x higher PL intensity, more than 4x longer lifetime and significantly reduced trap densities compared to the MAPB grown by ITC (47). In addition, the temperature profile plays an important role in the growth process and especially in the resulting crystal quality, and it should be tuned as a function of the seed quality (72).

For MAPI cystals, it is believed that synthesis at high temperature results in perovskite SC with inferior properties because of formation of $\mathrm{I}_{3}^{-}$traps acting as hole trapping centers, with major impact on mobility and lifetime $(27,53,54)$.

In order to grow MAPI SC at low temperature, an anti-solvent (ACN) can be added to the commonly used ITC solvent (GBL), thus reducing the solubility of the perovskite precursors. By tuning the anti-solvent concentration bulk SCs can be grown in a wide temperature range $\left(30^{\circ} \mathrm{C}-80^{\circ} \mathrm{C}\right)$, with best-quality crystals achieved at $60^{\circ} \mathrm{C}(68)$.

In alternative, the formation of $\mathrm{I}_{3}^{-}$traps can be prevented by halting the oxidation of $\mathrm{I}^{-}$into $\mathrm{I}_{3}^{-}$, by adding a strong reducing agent such as hypophosphorous acid $\left(\mathrm{H}_{3} \mathrm{PO}_{2}\right)$ to the ITC precursor solution (Figure 4d). A two orders of magnitude boost in photocurrent was obtained with $\mathrm{I}_{3}^{-}$-free MAPI SCs (54). 
a

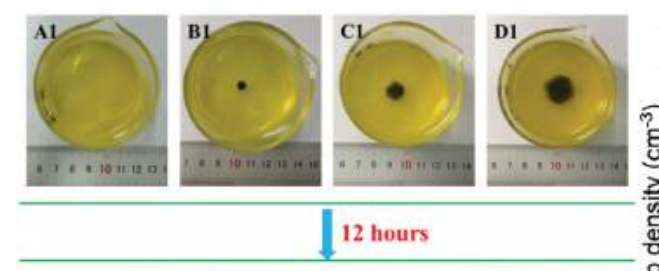

d
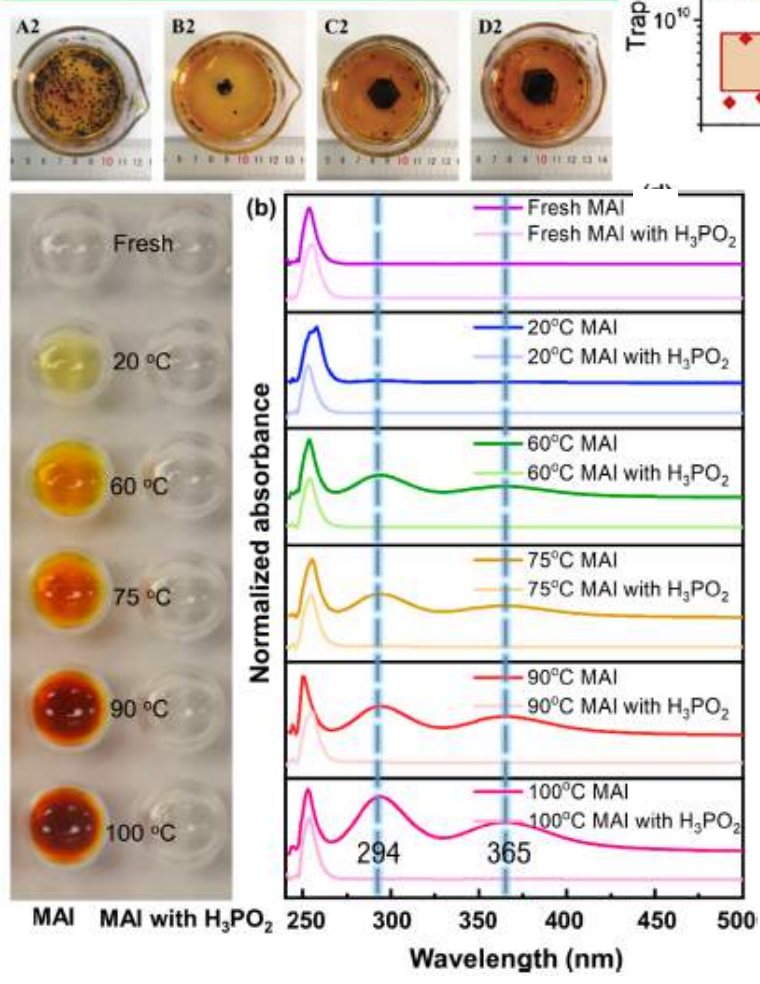

b Voltage (V)

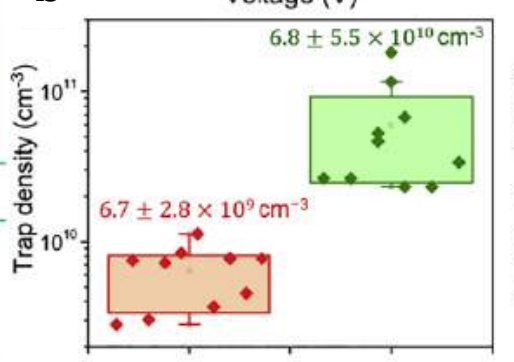

LTGC High Temp.
C

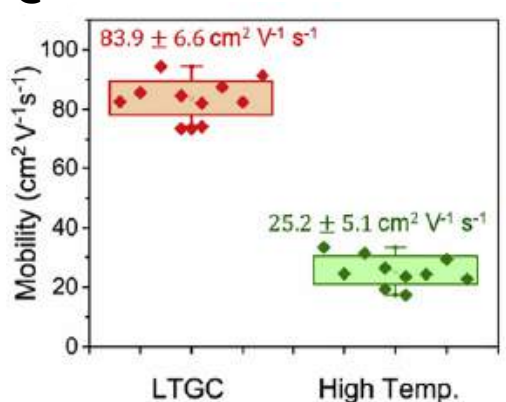

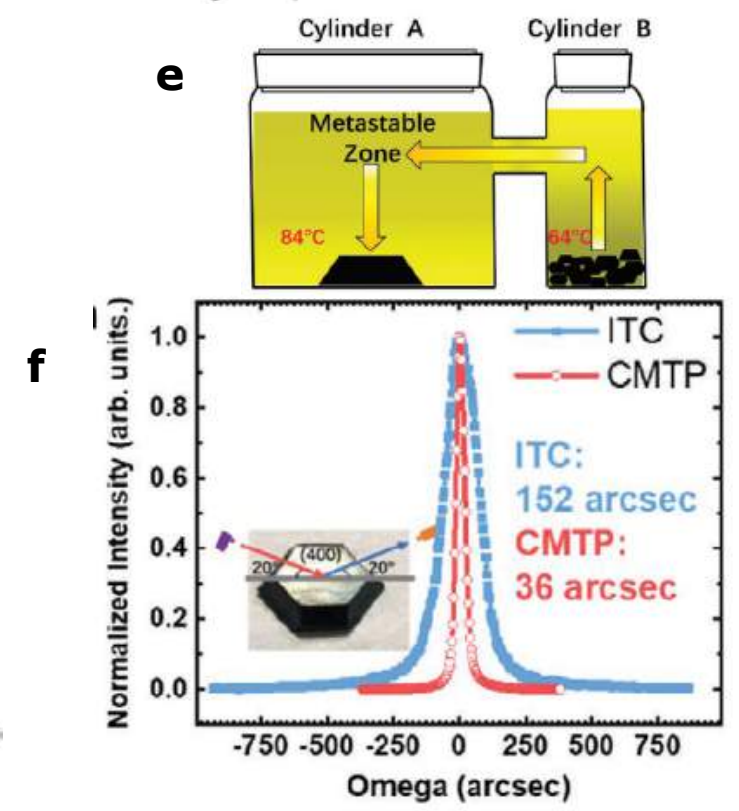

Figure 4 - MAPX-SC growth by ITC. a) Growth of MAPB-SC by successively using the growing crystal as a seed: A1. Clear precursor solution without seed. A2. The A1 after $12 \mathrm{~h}$ of growth at $100^{\circ} \mathrm{C}$. The $\mathrm{B} 1, \mathrm{C} 1$, and $\mathrm{D} 1$ are photos at initial stage when the seed crystal is placed into the fresh precursor solution. The B2, C2, and D2 are photos of the $\mathrm{B} 1, \mathrm{C} 1$, and $\mathrm{D} 1$ after $12 \mathrm{~h}$ of growth at $100^{\circ} \mathrm{C}$ (from ref.(66)) b) trap density and c) carrier mobility for both low-temperature (LTGC) and high-temperature (HT) grown crystals (from ref(70)). d) MAI with (right) and without (left) $\mathrm{H}_{3} \mathrm{PO}_{2}$ dissolved in GBL and heated at different temperature. Evidence of the $\mathrm{I}^{3-}$ formation is given by the color guide and UV-Vis absorption spectra (from ref.(54)) e) Schematic illustration of the continuous-mass transport (CMTP) process growth method f) X-ray rocking curves of MAPI-SC grown by ITC and CMTP showing (from ref.(7)).

Small variations in temperature induce important variation in precursors solubility and can impact crystal quality (72). Microwave radiation was employed to heat the precursor solutions in an attempt to improve temperature control and reproducibility, but didn't enable to obtain a fixed crystal growth rate (67). Wang et al. developed a continuous-mass transport process (CMTP) in order to keep the precursor concentration and thus the growth rate constant. By connecting a cylinder containing a seed immersed in a growth solution to a second cylinder kept at lower temperature and containing precursor material in the form of small crystals they were able to continuously feed the depleting growth solution as illustrated in Figure 4e. The growth solution was maintained at $84^{\circ} \mathrm{C}, 20^{\circ} \mathrm{C}$ above the feeding solution. This enabled to obtain a material with a dislocation density of $6.8 \cdot 10^{6} \mathrm{~cm}^{-2}$ compared to $2.2 \cdot 10^{8} \mathrm{~cm}^{-2}$ for crystals grown with the standard ITC method, evidenced by a 4 times narrower XRD peaks (Figure $4 \mathrm{f}$ ). In addition, mobility measurements confirmed the superiority of the CMTP crystals with values of $150.2 \mathrm{~cm}^{2} C^{-1} \mathrm{~s}^{-1}$ compared to $80.5 \mathrm{~cm}^{2} \mathrm{C}^{-1} \mathrm{~s}^{-1}$ for the ITC crystal (7).

A combination of the recent improvements in controlling temperature, growth rate and avoiding solvent contamination (32) might allow to grow high quality MAPX crystals at high speed with ITC techniques.

Although we chose to focus on MAPX SC, other types of perovskite highly promising for the solar industry were successfully grown as SC, and has been reviewed elsewhere $(17,25,30-43)$. For example formamidinium lead halide perovskite $\mathrm{FAPbX}_{3}$ and mixed cations perovskite $\mathrm{FA}_{(1-x)} \mathrm{MA}_{x} \mathrm{PbI}_{3}$, as well as mixed halides perovskites and even $\mathrm{FAPbBr}_{3-x} \mathrm{Cl}_{x}$ or $\mathrm{FAPb}_{1-x} \mathrm{Sn}_{x} \mathrm{Br}_{3}$, were succesfully grown by ITC (73-79). Lead free perovskite, $\mathrm{MASnI}_{3}$ and $\mathrm{FASnI}_{3}$ gaining increasing attention due to lead toxicity, were also successfully grown as $\mathrm{SC}$ by a top-seeded solution growth method (80). Finally, various methods to synthesize all-inorganic perovskite 
SC were also reported. These include the modified Bridgman growth method (81), vapor phase epitaxial growth (82), electro dynamic gradient (83) and even ITC through careful solvent engineering (84).

The optoelectronics properties of MAPX bulk SC are presented in section 3.

\section{Single crystal thin films synthesis}

Although research on bulk SC is very important to study properties and discover the ultimate performance of MAPX materials, solar cell devices are made from thin films. Therefore, in this section we will review the major strategies that are used to obtain MAPX SC thin films, that require some more degree of complexity compared to bulk SC, but are often based on similar approaches.

\subsection{Thin film growth on substrates}

\subsubsection{Space Limited Growth - SLG}

An effective approach to grow perovskite SC thin films is to physically block growth in one direction to enable 2D growth with methods developed to grow bulk SC (STL, SE, ITC, AVC). Crystals are grown between two parallel substrates clamped together and in contact with the precursor solutions. The thickness of the films can be controlled by adjusting the space between the two substrates (Figure 5). In most cases, one of the main challenges is to allow for fresh solution to reach the substrate during crystal growth to avoid precursors depletion, while maintaining the gap between the two substrates.

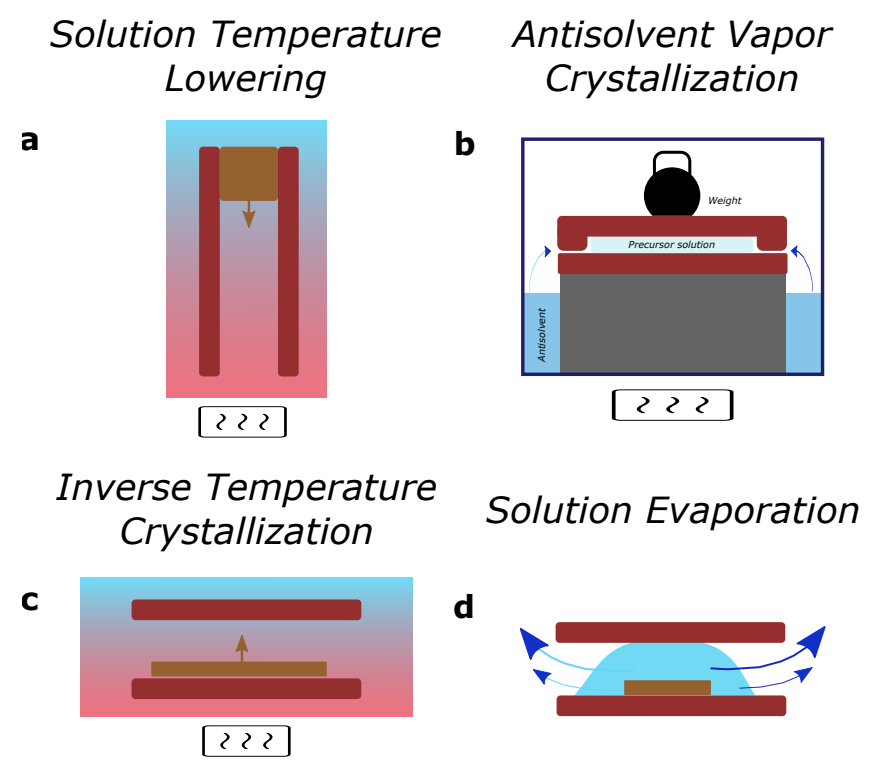

Figure 5 - Schematic representation of the SLG setups

STL When using the STL technique, the substrates are vertically dipped in a heated solution and the capillary pressure drives the solution between the substrates (Figure 5a). Crystallization occurs at the top, where the solution is colder. Chen et al reported (100) oriented MAPB thin films with a minimum thickness of $800 \mathrm{~nm}$, showing good air stability and trap densities as low as $4.8 \cdot 10^{10} \mathrm{~cm}^{-3}$ (85). In a similar fashion, 50 to 200 micrometer thick MAPI-SC thin films were directly grown between two parallel $\mathrm{FTO} / \mathrm{TiO}_{2}$, separated by a teflon spacer, by dipping the substrates vertically in the hot precursor solution. After peeling-off one of the substrates, the material was ready to be integrated in a solar cell through deposition of hole transport layer (HTL) and electrode (86).

SE Nguyen et al grew (110) MAPB thin films upon evaporation of a solvent by the cast capping method: the perovskite precursor solution was cast on a substrate and then capped with another substrate (Figure 5d). Slow solvent evaporation over the course of hours or days enabled the growth of $3.6 \mu \mathrm{m}$ thick millimiter sized SC $(87)$.

ITC The ITC technique is largely used to grow thin films. The precursor solution is typically heated from the bottom with a hot plate, and the substrate is either placed vertically or horizontally. An horizontal dipping configuration is often preferred to avoid crystallization preventing the free flow of precursors (Figure 5c) (88, 
89). U-shaped spacers can be used to better control the thickness of the film over a large area (Figure 6a). With this approach it was possible to obtain MAPB SC films over $120 \mathrm{~cm}^{2}$, with a thickness between $16 \mu \mathrm{m}$ and $100 \mu \mathrm{m}$ (Figure 6b). Such crystals exhibited trap density of $3.4 \pm 1.9 \cdot 10^{10} \mathrm{~cm}^{-3}$ as well as hole mobility of $40.7 \pm 7.7 \mathrm{~cm}^{2} \mathrm{~V}^{-1} \mathrm{~s}^{-1}$, and were successfully integrated in a solar cell $(89,90)$. The film thickness could be further reduced by applying pressure to reduce the gap between the two substrates, and enabled obtaining (001) oriented MAPB films with a thickness of $100 \mathrm{~nm}$, albeit at the cost of higher trap densities $\left(1.6 \cdot 10^{11} \mathrm{~cm}^{-3}\right)$ $(91)$.

The applicability of the ITC technique to SLG to grow MAPI films long remained challenging until seeded techniques were developed and enabled the formation of $50 \mu \mathrm{m}$ thick MAPI thin films (92). The surface chemistry of the substrate proved to have a crucial role in defining the material quality. By coating the substrate surfaces with a hydrophobic material to ensure good precursor diffusion between the two substrates high quality $10 \mu \mathrm{m}$ MAPI thin films were obtained. In addition, the outstanding properties (hole mobility of $121 \pm 15 \mathrm{~cm}^{2} \mathrm{~V}^{-1} \mathrm{~s}^{-1}$ and lifetime of $2.2 \mu \mathrm{s}$ ) enabled to achieve a PCE of of $17.8 \%$, a breakthrough in perovskite monocristalline solar cells (93). In addition to improving the diffusion of precursors solution, the substrate hydrophobicity contributed to decrease oxygen dandling bonds and therefore improved material quality (94).

The crystal quality could be further improved by lowering crystallization temperature via the addition of an anti-solvent, as described in section 1.2.3. Mixing propylene carbonate (PC) to the commonly used GBL solvent, it was possible to obtain a SC-MAPI solar cell with a record PCE of $21.9 \%$ (27).

AVC MAPI thin films synthesized by combining the AVC and space confined methods were recently obtained for the first time by using trichloroethane (TCE) as antisolvent (95). The film thickness could be controlled down to $300 \mathrm{~nm}$ by adjusting the loading weight of the one of the substrate (Figure $5 \mathrm{~b}$ ) which allowed the thin film to be integrated in a solar cell. However the synthesis was carried out at a temperature of $70^{\circ} \mathrm{C}$, arguably exposing the crystal structure to defects, while attempts to grow crystal at RT has remained so far unsuccessful $(27)$.

None of these techniques require lattice matching between the growing perovskite crystal and the substrate, allowing for on chip fabrication on a variety of substrates (silicon, PET, glass, quartz, mica, FTO or ITO, hole transport layers) for device integration. Many of those thin films were successfully integrated in solar cells $(27,86,89,92,93,96)$ (see section 5.3) and photodetectors $(88,90,91,96)$. More details on the topic of space-confined growth of metal halide perovskite crystal films can be found in a recent review by Li et al. (43). 

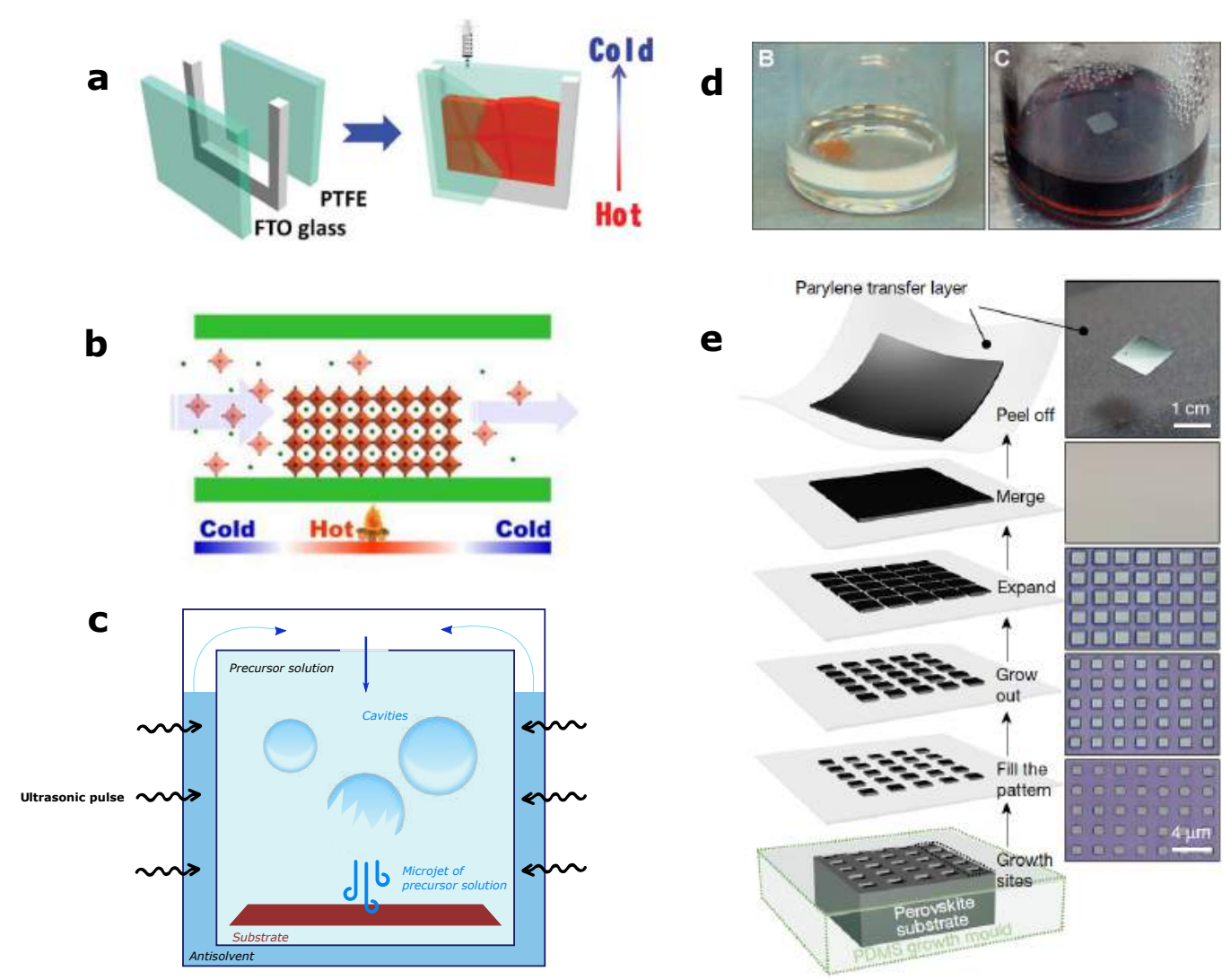

Figure 6 - Synthesis on thin films by the bottom-up method. Diagram of the SLG-ITC set-up without (a) (from ref. (90)) and with local heating (b) (from ref.(89); (c) Diagram of the CTAC process (97); (d) Photographs of (B) MAPB-SC and (C) MAPI-SC grown by the STITC method, floating on the solution surfaces (vial diameter is $15 \mathrm{~mm}$ ) (from ref.(98); (e) Lithography-assisted epitaxial-growth-and-transfer method. The optical images on the right correspond to the schematic representation on the left: epitaxial growth, merging and transferring processes (from ref.(99)).

\subsubsection{Cavitation triggered asymetrical crystallization - CTAC (97)}

An alternative method to obtain SC thin films without using physical boundaries to confine the growth, is the use of a pulsed ultrasonic-induced cavitation process to locally overcome the nucleation barrier and induce a flow of precursor solution toward the substrate (Figure 6c) in the presence of an anti-solvent. MAPB SC with the (001) facet parallel to the surface were obtained with a thickness ranging between $1 \mu \mathrm{m}$ and $60 \mu \mathrm{m}$. Importantly, ITO or FTO coated glass could be use as substrate, thus forming half cells ready to be integrated in solar devices upon crystallization (97).

\subsubsection{Epitaxial Growth}

MAPB and MAPI SC thin films can be prepared by epitaxy on both organic and inorganic preformed perovskite SC (99-101). Notably, Lei et al. developed a method that allowed them to separate an epitaxially grown layer of MAPI from the bulk SC (MAPI as well) on which it had been grown. A patterned polymer acting as growth mask was deposited on top of the bulk SC, so that epitaxial growth only occurred in areas not covered by the mask. Once the thickness of the growing SC exceeded that of the mask, growth occurred, not laterally restrained anymore, parallel to the substrate, leading to the formation of a SC thin film upon merging of adjacent epitaxial crystals, as depicted in Figure 6e. Because the substrate and the epitaxial layer were connected only in few locations, it was possible to peel off the thin film and transfer it to an arbitrary substrate. Such method allowed to synthesize SC thin films with thickness from $600 \mathrm{~nm}$ to $100 \mu \mathrm{m}$, that were successfully integrated in devices (99).

\subsubsection{Conversion Methods}

Perovskite thin film can also be synthesized through a conversion method. Fu et al. synthesized a $\mathrm{PbI}_{2}$ film on FTO by spin coating and transformed it into a (100) MAPI thin film upon reaction with MAI in solution (29). Such conversion can also be performed by a gas-solid reaction to form a MAPB thin film out of $\mathrm{PbBr}_{2}(102)$. 


\subsection{Free-standing thin films}

\subsubsection{Surface tension controlled ITC (98)}

Growing free-standing thin film is also of interest, as it allows to suppress any interaction with a substrate that could affect the growth and to further choose the contacts for the integration of the films in devices. Free standing $1 \mathrm{~cm}^{2}$ thin films with thickness ranging from $5 \mu \mathrm{m}$ (MAPI) to $10 \mu \mathrm{m}$ (MAPB) were successfully grown at the solution-air interface with the ITC technique by using a solvent with high surface tension coefficient such as DMSO (Figure 6d). Nucleation occurred at the solution surface and the nucleus grew into a crystal along the solution surface until it reached a critical size when surface tension could no longer keep the crystal afloat (98). Such method allowed to form MAPI thin film with (002) or (110) facets parallel to the surface, depending on the molar ratio $\mathrm{MAI} / \mathrm{PbI}_{2}$, as well as MAPB thin films with the (001) facet parallel to the surface and trap densities of $5.3 \cdot 10^{11} \mathrm{~cm}^{-3}$ comparable to SC thin films grown by other methods.

\subsubsection{From Bulk Crystals}

Top-down approaches can be employed to obtain millimeter or micrometer-thin perovskite wafers (6, 62, 103), in a similar fashion to SC silicon ingots, with potential impact on industrialization. Lv et al. used a diamond wire to slice bulk single crystals grown by STL (for MAPI) or ITC (for MAPB, MAPC) into thin films of arbitrary crystal orientation (103). Mechanical polishing and chemical etching can be used to further reduce the film thickness down to $15 \mu \mathrm{m}$, while maintaining a surface area above $10 \mathrm{~mm}^{2}$, as summarized in Figure 7a (103). Micro-crystalline precipitation as a consequence of etching was shown to be prevented by placing SC on a rotating stand to spread the etching solution uniformly on the SC wafer while removing rapidly the residual etching solution from the surface (104).

Alternatively, sandpaper was used to thin down MAPI and MAPB bulk crystals grown by ITC, followed by additional lapping with calcined alumina sheets to reduce surface roughness down to $0.3 \mu \mathrm{m}$ (Figure7b) (105).
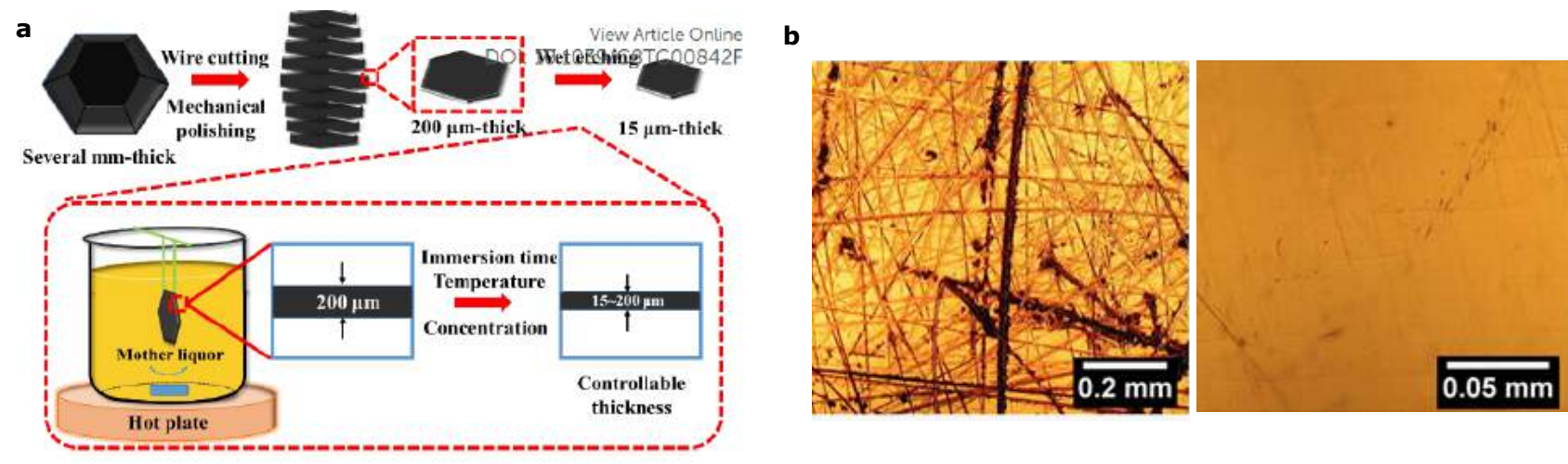

Figure 7 - Synthesis of thin films by the bottom-up method. a) Diagram of the top-down method for MAPB-SC thin films (from ref.(103)); b) left image is the MAPB-SC surface after manual sandpaper grounding and lapping sheets and right image is the MAPB-SC after machine grinding (from ref.(105)).

The top down approach is a general method for preparing thin films of controllable thickness and orientation, allowing to expose other facets than the (100) typically obtained by the SLG technique. In addition the resulting sliced films are stress-free and can have a large lateral size, thus facilitating integration in different device geometries. However, obtaining thin films with a thickness smaller than the diffusion length is challenging, thus hampering their use in vertical solar cells.

\subsection{Patterned Thin Films and crystal arrays}

Being able to synthesize patterned perovskite SC thin films is highly desirable as it can (i) support integrated light trapping schemes and nanophotonics concepts (106), (ii) enable to develop creative device architectures to limit carrier recombination by reducing the distance traveled by minority carriers before being collected (107-109).

However, patterning MAPX SC thin films is complex, as one has to combine crystallization and patterning techniques altogether. Typically, the substrates used to confine the growth are patterned beforehand, or as an alternative the precursor solution is selectively deposited on the substrate. These approaches are discussed below in detail. 


\subsubsection{Confined growth}

Blade Coating Blade coating is based on the evaporation method. A precursor solution is cast on a substrate and a blade, placed above it, moves laterally, allowing to drag the solution over the whole substrate. As the solution evaporates, perovskite precursors tend to accumulate at the contact line, dragged by the evaporationinduced convective flow, eventually triggering nucleation on the substrate. By simply casting MAPI precursor solution on a hot unpatterned substrate, Deng et al. were able to form MAPI single crystalline nanowires arrays oriented parallel to the blade direction as the blade moved along (110).

The use of pre-patterned substrate enables to further control the crystal shape, as shown in Figure 8a. This enabled for the fabrication of SC arrays (Figure 8b) from an ITO substrate, patterned with periodic parallel microchannels $1 \mu \mathrm{m}$ deep and $2 \mu \mathrm{m}$ wide. The array had an average carrier lifetime of $175 \mathrm{~ns}$ (MAPI), and were successfully integrated in vertical photo-detector arrays for high-resolution imaging (111).

Extrusion SC MAPB nanowires arrays were synthesized by Oener et al. by extrusion of perovskite growth solution $\left(\mathrm{MABr}, \mathrm{PbBr}_{2}\right)$ through a anodized aluminum oxide $(\mathrm{AAO})$ template presenting nanopore arrays. The precursor solution was dropcasted on the AAO substrate, and by application of a pressure gradient the solution was forced into the nanopores. Confinement of the solution in the nanopores and faster evaporation at the pore exit resulted on the formation of freestanding MAPB SC nanowires in less than a minute (Figure 8g). A maximum PLQY (photoluminescence quantum yield) of $29.5 \%$ was measured (112), suggesting high material quality.

Liquid Knife Feng et al. formed MAPB single plates arrays with the liquid knife method. They designed a structured silicon template with micropillars presenting a lyophilic top with the help of photolitography to act as the liquid knife. This structured template was then drawn close enough to a precursor solution for a liquid meniscus to be formed on top of each pillar, thus splitting the perovskite precursor liquid into regular domains (Figure 8f). Upon solvent evaporation each domain transformed into a SC, yielding a regular array of $0.9 \mu \mathrm{m}$ thick square microplates. They showed that the size could be tuned by controlling the volume of liquid domains (113).

Ostwald ripening assisted photolitography - ORAP Wu et al. used a combination of the blade coating and the liquid knife method. A perovskite solution was dragged on a substrate presenting difference in wettability achieved by photolithography, allowing to divide the solution into pre-defined domains (Figure 8c). The perovskite solution domains were successively crystallized in IPA atmosphere and redissolved in DMF through an Ostwald ripening assisted crystallization process which allowed smaller crystals to dissolve and redeposit onto the larger one (Figure $8 \mathrm{~d}$ and e). Microplates with long range arrangement of average thickness of $3 \mu \mathrm{m}$ were obtained on top of $\mathrm{TiO}_{2}$ and successfully integrated in micro-arrays solar cells with PCE of 7.84\% (114).

Roll printing technique (115) Thin patterned MAPI SC-films with mobilities of $\sim 46 \mathrm{~cm}^{2} \mathrm{~V}^{-1} \mathrm{~s}^{-1}$ were reported by Lee et al. through a method based on instant crystallization of a perovskite ink solution on a hot substrate. The ink was continuously transferred to the substrate through a rolling mold, allowing the crystals to grow as new material was deposited, while simultaneously confining the growth on the substrate. Patterning of the perovskite film was achieved by wrapping the rolling mold in a flexible polydimethylsiloxane (PDMS) substrate with wide $(10 \mu \mathrm{m})$ and shallow $(200 \mathrm{~nm}$ deep) channels, determining thickness and lateral size of the films (Figure 8h). The length of the film was controlled by the distance traveled by the rolling mold and was in the order of a few centimeters. Such crystal arrays were shown to be compatible with integration in lateral devices, as will be discussed later (115). 
a

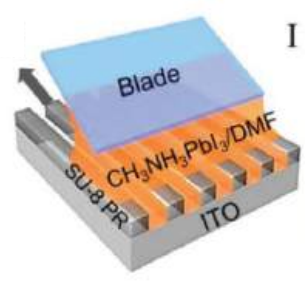

C

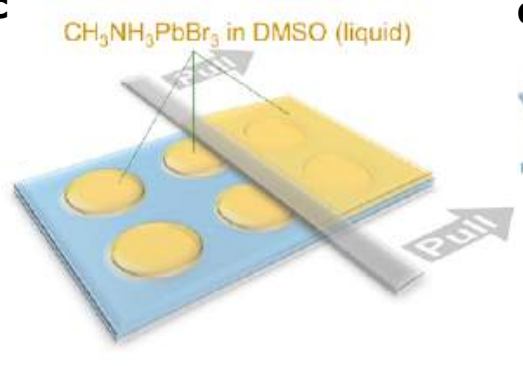

II

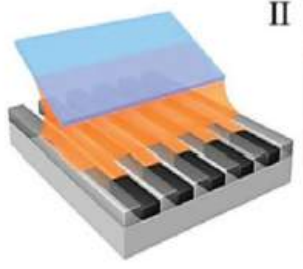

b

d

$\mathbf{f}$

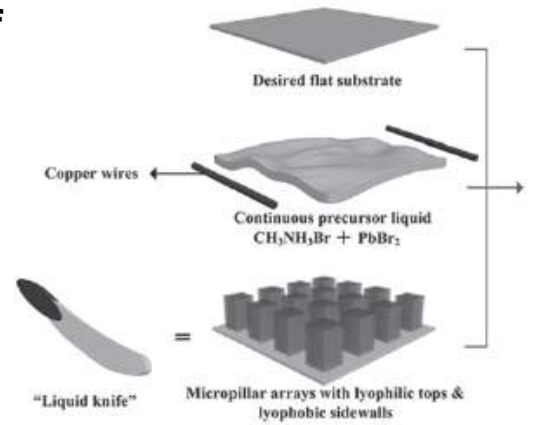

g

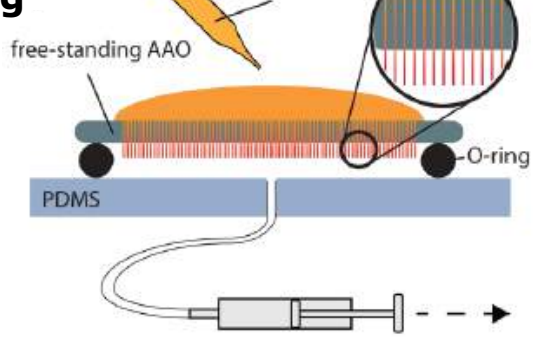

III
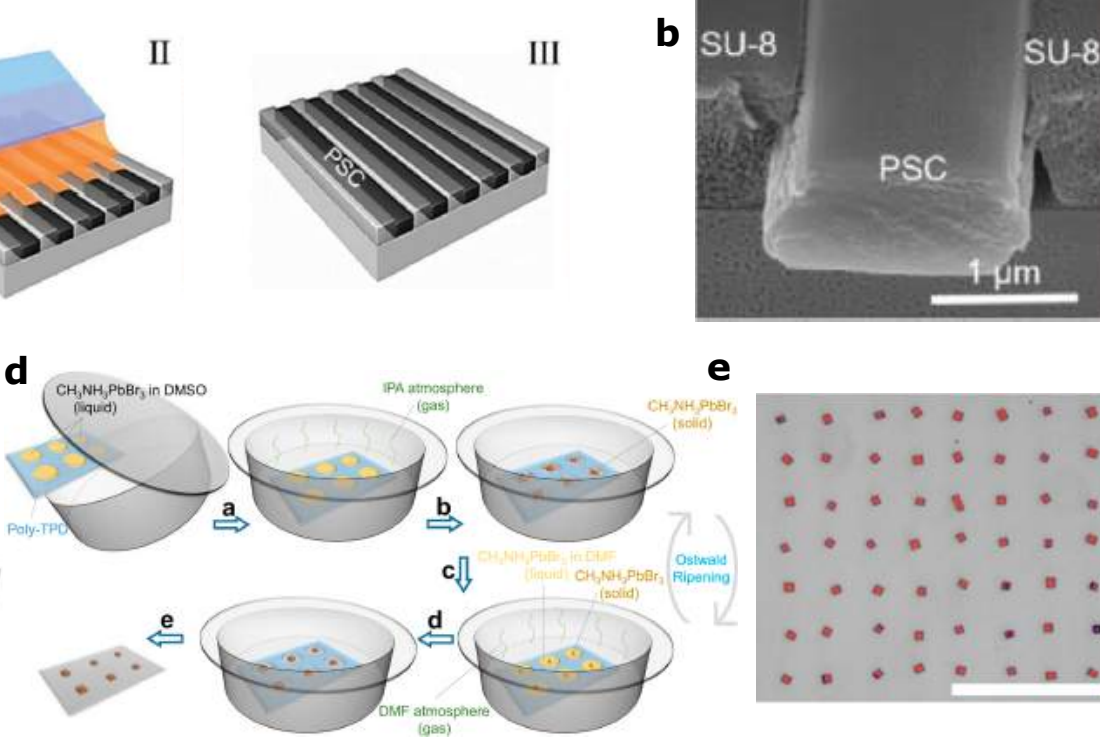

e
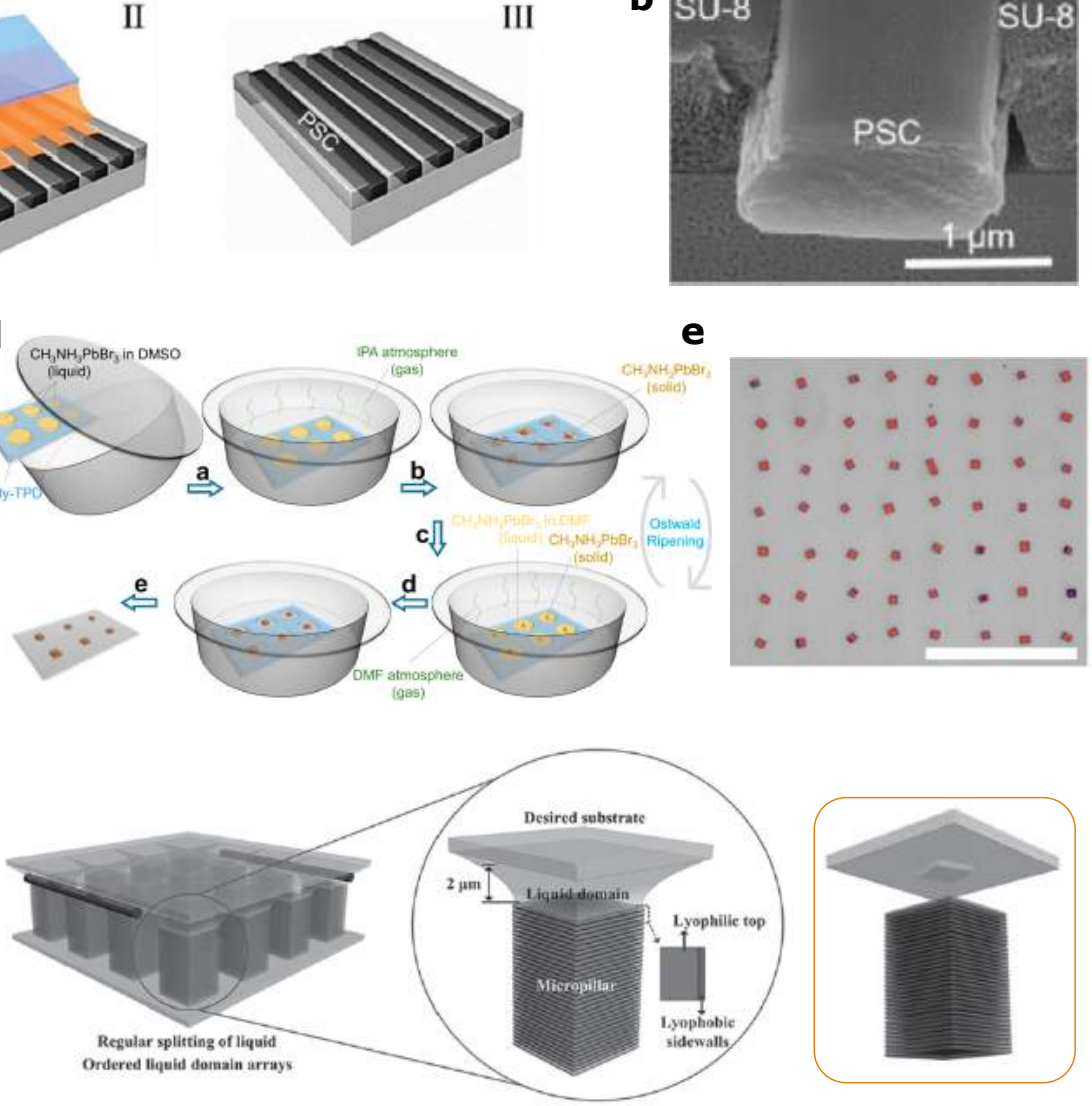

h

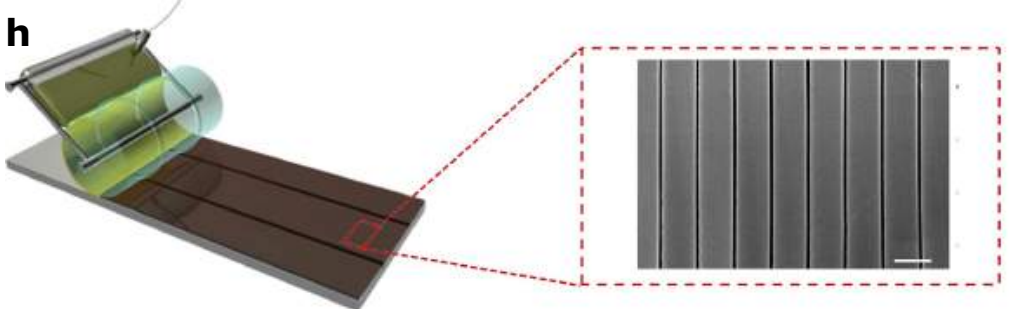

Figure 8 - Growth of patterned SC thin films and arrays in confined environments. Blade coating. a) Diagram of blade coating SC arrays using microchannel confined crystallization (MCC) strategy b) Cross-sectional SEM image of one single MAPI-SC grown by the MCC method (a and b from ref.(111)) ORAP. c) Diagram of first step: a wettability-assisted blade-coating d) Diagram of step 2: an Ostwald ripening assisted crystallization process e) Optical microscopic picture of the resulting SC array (scale bar: $100 \mu \mathrm{m})$ (c, d and e from ref.(114)) Liquid knife. f) Diagram of the method: micropillar arrays split the precursor solution in regular domains that eventually form SC (from ref.(116)) Extrusion. g) Perovskite nanowire extrusion scheme (from ref.(112) Roll printing. h) Schematic illustration of the technique using a patterned rolling mold to provide precursor inks and confined the growth and SEM image of the resulting SC patterned thin film consisting of 10-mm-wide strips with 400-nm-wide spacing (from ref.(115)).

\subsubsection{Epitaxial Growth}

Epitaxy on a pre-patterned substrate proved effective to obtain patterned SC thin films. The MAPB substrate, that is moisture-sensitive, was first protected by a triple layer of Parylene- $\mathrm{C} / \mathrm{Cr} / \mathrm{Au}$ to avoid damage or dissolution. Local etching of this triple layer eventually led to the opening of sites for epitaxial growth (Figure 9b). Trap densities as low as $5.29 \cdot 10^{10} \mathrm{~cm}^{-3}$ as well as slow lifetime components of $93.60 \mathrm{~ns}$ for a $5 \mu \mathrm{m}$ thick layer were reported. Importantly, by playing with the growth conditions such as time and temperature, crystals with different morphology and orientation could be obtained, as illustrated in Figure 9a. The process proved compatible with the realization of LED arrays. A PEDOT:PSS layer was added on top of the triple layer prior to perovskite growth in solution to act as a hole transporting layer (HTL). A top electrode was deposited after the epitaxial growth, while $\mathrm{Cr} / \mathrm{Au}$ acted as the bottom electrode. The resulting device showed better performances compared to a PC device (117). 
a

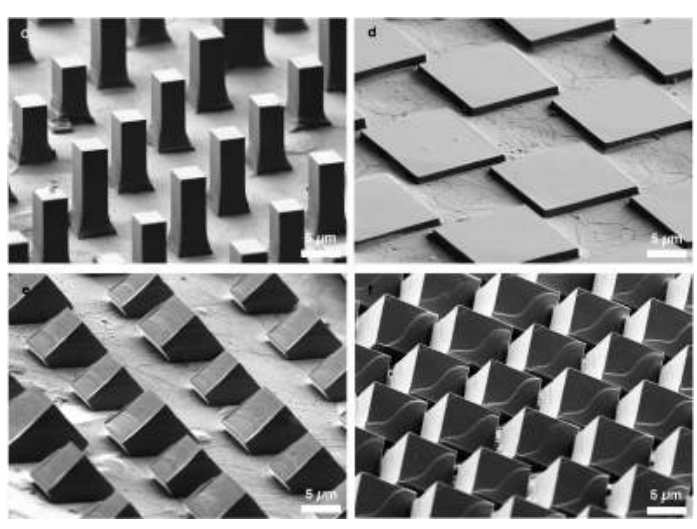

b

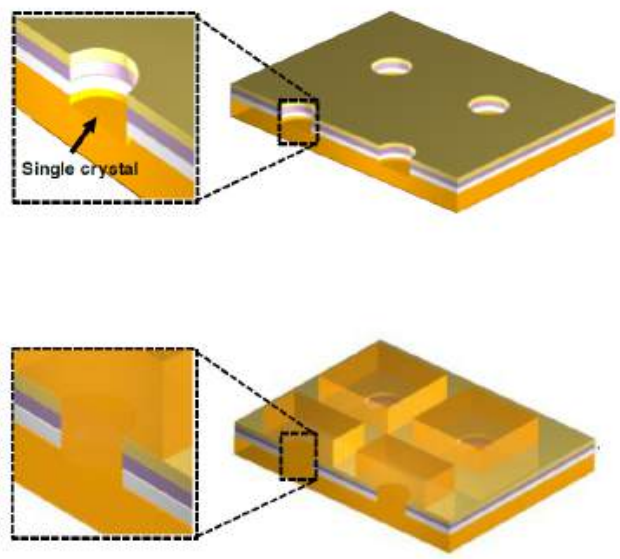

Figure 9 - Epitaxial growth of patterned thin films. a) SEM images showing the controllable homoepitaxial growth of MAPB-SC by epitaxy b) Diagram of the growth process (a and b from ref.(117)).

\subsubsection{Inkjet Printing}

The other approach to growing patterned thin films or crystal arrays is to deposit precursors in predefined locations. Inkjet printing, by allowing to deposit ink droplets on a substrate according to precise preset patterns, enables patterning thin films without using top-down processes such e-beam or UV-lithography.

By depositing a controlled amount of perovskite precursor solution $\mathrm{Gu}$ et al. were able to grow arrays of MAPB SC microplates on low adhesion materials through solvent evaporation. Interestingly, they observed that substrate adhesion affected the contact line and influenced the formation of the perovskite single crystal. Low surface energy substrates such as PDMS were essential to forming microsized square perovskite single crystal microplate arrays (Figure 10a) (118). By reducing the amount of perovskite precursor solution, small seeds can be obtained as the solvent evaporated (Figure 10b). These seeds were further grown into well arranged perovskite microplates that could be printed to another substrate (119). This approach could have important implications for the realization of tandem solar cells. 


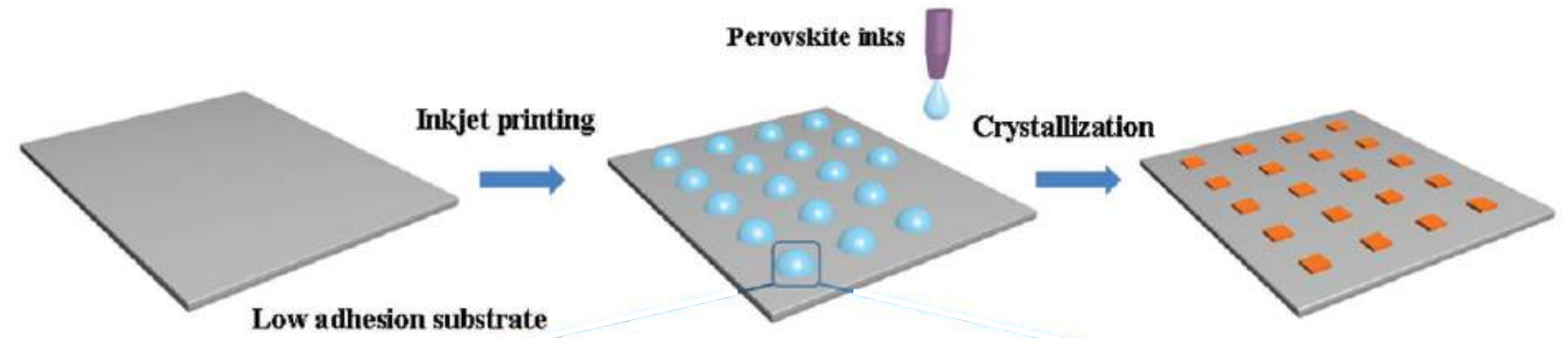

b Seeded synthesis of MAPB microplates arrays

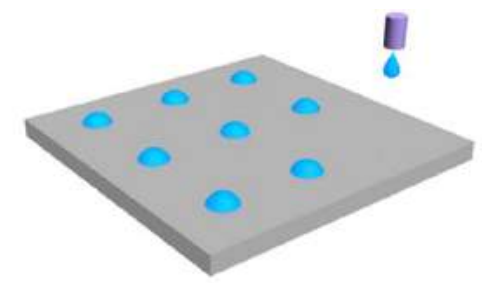

Droplet printing

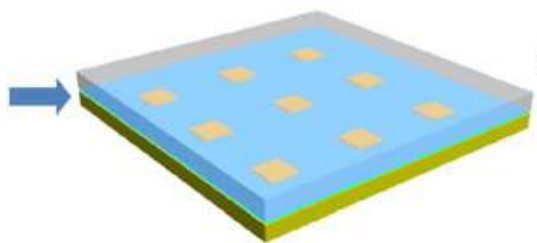

Film growth

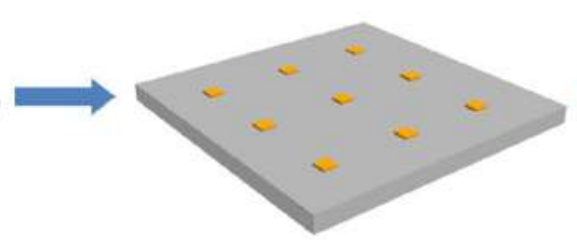

Perovskite seed stamp

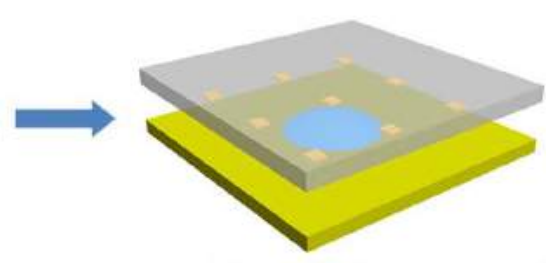

Perovskite solution covered with the seed stamp

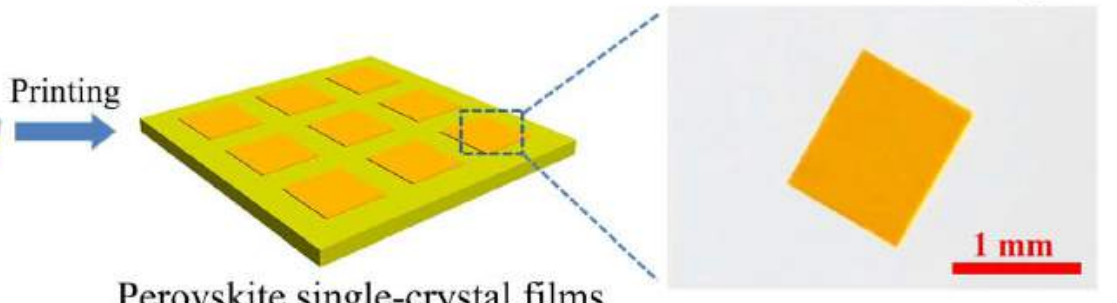

Figure 10 - Preparation of the SC microplate arrays by inkjet printing. Diagram of a) the one-step method (from ref.(118) b) The seeded method. The prepared seed stamp obtained by inkjet printing is placed on the target substrate with the perovskite solution. SC arrays form as the seed grow upon solvent evaporation (from ref.(119)).

\subsection{Summary of the growth methods}

All the techniques developed to grow bulk SC, TF and patterned TF are summarized in Figure 11:

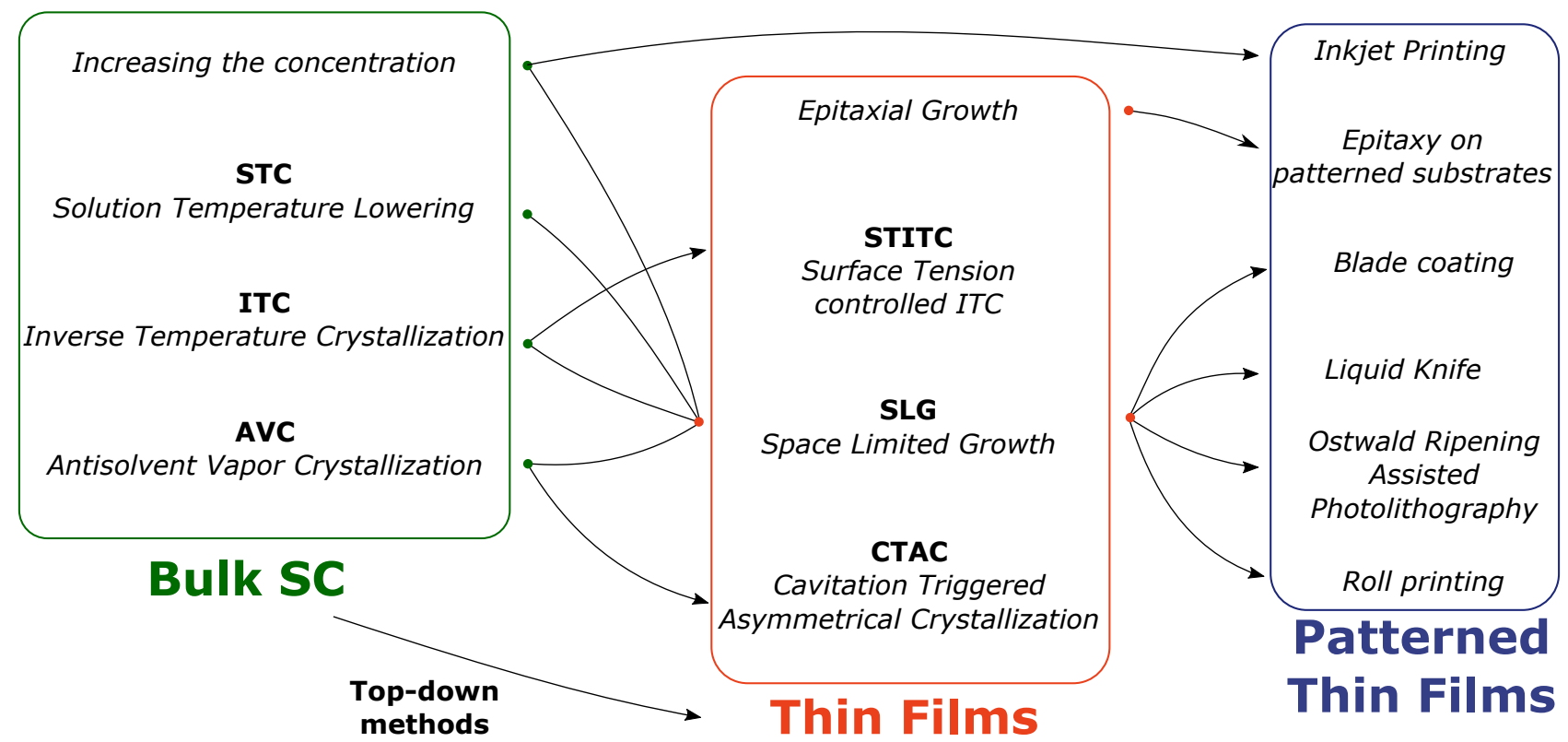

Figure 11 - Summary of all the fabrication techniques, from bulk to patterned thin films 


\section{Optoelectronic properties}

The optoelectronic properties of bulk SC and SC thin films are presented in the tables below, for different synthesis methods. The characterization techniques will be presented in section 3.3. 


\subsection{MAPB}

Bulk single crystals

\begin{tabular}{|c|c|c|c|c|c|c|}
\hline Method & $\begin{array}{l}\text { Growth } \\
\text { temperature } \\
{ }^{\circ} \mathrm{C}\end{array}$ & $\begin{array}{l}\text { Trap density }{ }^{\dagger} \\
\mathrm{cm}^{-3}\end{array}$ & $\begin{array}{l}\text { Carrier mobility } \\
\mathrm{cm}^{2} \mathrm{~V}^{-1} \mathrm{~s}^{-1}\end{array}$ & $\begin{array}{l}\text { Slow lifetime } \\
\text { component } \\
\text { ns }\end{array}$ & $\begin{array}{l}\text { Diffusion length } \\
\mu \mathrm{m}\end{array}$ & Ref \\
\hline \multirow{3}{*}{ LDSC } & 50 & - & - & - & - & (44) \\
\hline & 58 & - & - & - & - & (45) \\
\hline & RT & $\begin{array}{l}6.5 \pm 0.4 \cdot 10^{9}\left(\mathrm{e}^{-}\right) \\
4.4 \pm 0.2 \cdot 10^{9}\left(\mathrm{~h}^{+}\right)\end{array}$ & - & $997^{* *}$ & - & $(47)$ \\
\hline \multirow[b]{2}{*}{ AVC } & $\mathrm{RT}$ & - & - & - & - & $(57)$ \\
\hline & $\mathrm{RT}$ & $5,8.10^{9}$ & $\begin{array}{c}115^{\dagger \dagger} \\
20-60^{\ddagger}\end{array}$ & $337^{*}$ & 17 & $(56)$ \\
\hline \multirow{3}{*}{ ITC } & $25 \rightarrow 60$ & $6,7 \pm 2,8.10^{9}$ & $83,9 \pm 6 \ddagger\left(\mathrm{h}^{+}\right)$ & $897 \pm 113$ ** & $13,8 \pm 0,7$ & $(70)$ \\
\hline & 80 & $3,0.10^{10}$ & $24^{\dagger}$ & $300 \pm 26$ * & 4,3 & (69) \\
\hline & 100 & $\begin{array}{l}2,6.10^{10}\left(\mathrm{~h}^{+}\right) \\
1,1.10^{11}\left(\mathrm{e}^{-}\right)\end{array}$ & $4,36^{\ddagger}$ & - & - & (66) \\
\hline
\end{tabular}

\section{Single crystal thin films}

\begin{tabular}{|c|c|c|c|c|c|c|c|}
\hline Method & $\begin{array}{c}\text { Temperature } \\
{ }^{\circ} \mathrm{C} \\
\end{array}$ & $\begin{array}{c}\text { Minimal } \\
\text { thickness } \\
\mu \mathrm{m}\end{array}$ & $\begin{array}{c}\text { Trap } \\
\text { density } \\
\mathrm{cm}^{-3}\end{array}$ & $\begin{array}{c}\text { Carrier } \\
\text { mobility } \\
\mathrm{cm}^{2} \mathrm{~V}^{-1} \mathrm{~s}^{-1}\end{array}$ & $\begin{array}{c}\text { Slow lifetime } \\
\text { component } \\
\text { ns }\end{array}$ & $\begin{array}{l}\text { Diffusion } \\
\text { length } \\
\mu \mathrm{m}\end{array}$ & Ref \\
\hline \multirow{7}{*}{ SLG } & 90 & 0.8 & $4.8 \cdot 10^{10}$ & $15.7^{\dagger}$ & - & - & (85) \\
\hline & $60-80$ & 0.6 & - & - & $301^{* *}$ & - & (96) \\
\hline & 120 & 100 & $3.4 \pm 1.9 \cdot 10^{10}$ & $\begin{array}{l}40.7 \pm 7.7^{\dagger}\left(\mathrm{h}^{+}\right) \\
27.3 \pm 7.1^{\dagger}\left(\mathrm{e}^{-}\right)\end{array}$ & $390 \pm 21^{* *}$ & $\begin{array}{l}5.2\left(\mathrm{~h}^{+}\right) \\
6.4\left(\mathrm{e}^{-}\right)\end{array}$ & (90) \\
\hline & 90 & 16 & $2.5 \cdot 10^{10}$ & $23.7^{\dagger}$ & $\begin{array}{c}122.4^{* *} \\
\text { on FTO }\end{array}$ & - & (89) \\
\hline & 80 & 0.1 & $1.6 \cdot 10^{11}$ & $60^{\ddagger}$ & - & - & (91) \\
\hline & 120 & 3.6 & - & - & - & - & (87) \\
\hline & 65 & 200 & - & - & - & - & (100) \\
\hline CTAC & RT & 1 & $10^{11}$ & - & - & - & (97) \\
\hline \multirow{2}{*}{$\begin{array}{c}\text { Free } \\
\text { standing }\end{array}$} & 100 & 5 & $5.3 \cdot 10^{11}$ & - & - & - & (98) \\
\hline & 140 & $\mathrm{~nm}$ & $1.17 \cdot 10^{10}$ & - & - & - & (102) \\
\hline $\begin{array}{l}\text { Liquid } \\
\text { Knife }\end{array}$ & $\mathrm{RT}$ & 0.9 & - & - & $<20 \mathrm{ps}{ }^{\circledast} \circledast$ & - & (113) \\
\hline $\begin{array}{c}\text { Blade } \\
\text { Coating }\end{array}$ & RT & 3 & - & - & - & - & (114) \\
\hline $\begin{array}{c}\text { Inkjet } \\
\text { Printing }\end{array}$ & $\mathrm{RT}$ & 2 & - & - & $391^{* *}$ & - & (119) \\
\hline \multirow{2}{*}{$\begin{array}{l}\text { Epitaxial } \\
\text { Growth }\end{array}$} & 40 & 5 & $5.29 \cdot 10^{10}$ & $82^{\ddagger}$ & $93.60^{* *}$ & - & (117) \\
\hline & $80-160$ & 0.6 & - & $10-40^{\dagger \dagger}$ & $300-600^{* *}$ & $5-7$ & (99) \\
\hline
\end{tabular}

Table 2 - MAPB bulk and thin films SC optoelectronic properties 


\subsection{MAPI}

Bulk single crystals

\begin{tabular}{|c|c|c|c|c|c|c|}
\hline Method & $\begin{array}{l}\text { Growth } \\
\text { temperature } \\
{ }^{\circ} \mathrm{C} \\
\end{array}$ & $\begin{array}{l}\text { Trap density }{ }^{\dagger} \\
\mathrm{cm}^{-3}\end{array}$ & $\begin{array}{l}\text { Carrier mobility } \\
\mathrm{cm}^{2} \mathrm{~V}^{-1} \mathrm{~s}^{-1}\end{array}$ & $\begin{array}{l}\text { Slow lifetime } \\
\text { component } \\
\text { ns }\end{array}$ & $\begin{array}{l}\text { Diffusion length } \\
\mu \mathrm{m}\end{array}$ & Ref \\
\hline \multirow{4}{*}{ STL } & $65 \rightarrow 40$ & - & - & - & - & (49) \\
\hline & $108 \rightarrow 60$ & $3,8 \pm 1,1.10^{9}$ & $121 \pm 20^{\dagger}$ & - & - & $(51)$ \\
\hline & $75 \rightarrow ?$ & $\begin{array}{ll}3,6.10^{10} & \left(\mathrm{~h}^{+}\right) \\
4,5.10^{10} & \left(\mathrm{e}^{-}\right)\end{array}$ & $\begin{array}{c}164 \pm 25^{\dagger}\left(\mathrm{h}^{+}\right) \\
24,8 \pm 4,1^{\dagger \dagger}\left(\mathrm{e}^{-}\right)\end{array}$ & $\begin{array}{c}8,2 \pm 5.10^{4 * * *} \\
95 \pm 8.10^{4 \circledast}\end{array}$ & 175 & $(5)$ \\
\hline & $100 \rightarrow 60$ & - & - & - & - & $(50)$ \\
\hline $\mathrm{AVC}$ & $\mathrm{RT}$ & $3.3 \cdot 10^{10}$ & $2,5^{\dagger}$ & 1032 * & 8 & (56) \\
\hline \multirow{5}{*}{ ITC } & 100 & $5,9 \pm 1,8.10^{9}$ & $74 \pm 12^{\dagger}$ & - & - & (51) \\
\hline & 110 & $1,4.10^{10}$ & $67,2^{\dagger}$ & $570 \pm 69 *$ & 10 & (69) \\
\hline & 100 & $2,1.10^{10}$ & $41 \ddagger$ & - & - & (6) \\
\hline & 100 & $\begin{array}{l}1,8 \cdot 10^{9}\left(\mathrm{~h}^{+}\right) \\
4,8 \cdot 10^{10}\left(\mathrm{e}^{-}\right)\end{array}$ & $34 \ddagger$ & - & - & (66) \\
\hline & 84 & - & $150^{\dagger \dagger}$ & - & - & (7) \\
\hline
\end{tabular}

\section{Single crystal thin film}

\begin{tabular}{|c|c|c|c|c|c|c|c|}
\hline Method & $\begin{array}{c}\text { Temperature } \\
{ }^{\circ} \mathrm{C} \\
\end{array}$ & $\begin{array}{c}\text { Minimal } \\
\text { thickness } \\
\mu \mathrm{m}\end{array}$ & $\begin{array}{c}\text { Trap } \\
\text { density } \\
\mathrm{cm}^{-3}\end{array}$ & $\begin{array}{c}\text { Carrier } \\
\text { mobility } \\
\mathrm{cm}^{2} \mathrm{~V}^{-1} \mathrm{~s}^{-1}\end{array}$ & $\begin{array}{c}\text { Slow lifetime } \\
\text { component } \\
\text { ns }\end{array}$ & $\begin{array}{c}\text { Diffusion } \\
\text { length } \\
\mu \mathrm{m}\end{array}$ & Ref \\
\hline \multirow{8}{*}{$\mathrm{SLG}$} & 120 & 50 & - & - & $206^{* *}$ & - & $(86)$ \\
\hline & 100 & & - & - & $84000^{\circledast}$ & - & $(85)$ \\
\hline & 120 & 10 & - & $\begin{array}{c}121 \pm 15^{\dagger}\left(\mathrm{h}^{+}\right) \\
36,8 \pm 3,7^{\dagger}\left(\mathrm{e}^{-}\right)\end{array}$ & $2200 \pm 5000^{* * *}$ & $\begin{array}{l}24.7\left(\mathrm{~h}^{+}\right) \\
13.6\left(\mathrm{e}^{-}\right)\end{array}$ & $(93)$ \\
\hline & 110 & 150 & $6 \cdot 10^{8}$ & $39.6^{\ddagger}$ & - & - & $(88)$ \\
\hline & 140 & 50 & - & - & - & - & $(92)$ \\
\hline & 90 & 20 & - & - & $164^{* *}$ & - & $(27)$ \\
\hline & 130 & 20 & - & - & $42^{* *}$ & - & $(27)$ \\
\hline & 70 & 0.3 & $2.23 \cdot 10^{11}$ & 142 & 132 & - & $(95)$ \\
\hline \multirow{2}{*}{ Conversion } & 110 & 5 & - & - & - & - & (98) \\
\hline & $\mathrm{RT}$ & $\mathrm{nm}$ & - & - & $3^{*}$ & - & $(29)$ \\
\hline $\begin{array}{c}\text { Roll } \\
\text { Printing }\end{array}$ & 180 & 0.2 & - & $45.64^{\dagger}$ & - & - & $(115)$ \\
\hline Blade & 100 & $\mu \mathrm{m}$ & - & - & - & - & $(110)$ \\
\hline Coating & 35 & 1 & $2 \cdot 10^{9}$ & - & $151^{\circledast \circledast \circledast}$ & - & (111) \\
\hline
\end{tabular}

Table 3 - MAPI bulk and thin films SC optoelectronic properties 
$\dagger$ I-V measurements $/{ }^{\dagger \dagger}$ Time of Flight (ToF) / ${ }^{\ddagger}$ Hall measurement / ${ }^{*}$ Transient absorption / ${ }^{* *}$ Time resolved photoluminescence $/^{* * *}$ Transient Photovoltaic (1 sun) ${ }^{\circledast}$ Impedance spectroscopy (1 sun) ${ }^{\circledast \circledast}{ }^{\circledast}$ fluorescence lifetime imaging microscopy ${ }^{\circledast \circledast \circledast}{ }^{\circledast}$ transient state fluorescence spectrometer

From the data reported in the tables, one can draw the following conclusions:

1. MAPX SC typically have trap densities in the range $10^{9}-10^{11} \mathrm{~cm}^{-3}$, that is even lower than other monocristalline materials such as $\mathrm{Si}\left(10^{13}-10^{14} \mathrm{~cm}^{-3}\right)$, CIGS $\left(10^{13} \mathrm{~cm}^{-3}\right)$ or CdTe $\left(10^{11}-10^{13} \mathrm{~cm}^{-3}\right)$. For comparison, PC MAPX typically have trap densities in the order of $1.10^{17} \mathrm{~cm}^{-3}$. This, in combination with relatively good lifetime and mobility, shows the potential of MAPX SC for optoelectronic applications (47).

2. It is not easy to identify the optimal method for MAPX SC synthesis, as the optoelectronic properties reported for a given method span across a wide range. This is possibly due to differences in conditions and techniques used to assess them, and will be discussed in section 3.3. It is worth to notice that for MAPB SCs grown via the ITC technique, better properties are obtained with low temperature growth (70), approaching those obtained for LDSC crystals grown at room temperature $(47,56)$. Likewise, hightemperature growth is detrimental for MAPI SC beacuse it promotes the formation of $\mathrm{I}_{3}^{-}$traps $(47,70)$.

3. The relatively small diffusion length $(<20 \mu \mathrm{m}$ for most reports) disqualifies the implementation of bulk SC (that are typically at the millimiter scale) in traditional solar cells architecture (111). This implies the necessity to employ thin films, that however in most reports shows inferior properties compared to bulk SC, but better than PC. This will be discussed in section 6 .

4. SC films exhibit far better properties than PC films, but are often thicker (53). It is therefore essential to develop ways to further reduce the thickness of the films to match diffusion length, that is required to build efficient perovskite SC solar cells. This is especially true for MAPI, that is more promising for solar cells applications due to the more suitable band-gap, for which very few reports of sub-micron films are available.

5. It is important to adapt the latest low temperature growth techniques developed to grow bulk crystal to thin films and patterned thin films. Despite the drawbacks discussed in section 1.2.3, MAPB SLG thin films are often grown by the ITC method $(85,91,96)$, whereas for patterned thin films most strategies are based on a fast evaporation, even though bulk crystal studies revealed that controlled growth kinetic as well as low temperature growth was important to ensure good optoelectronic properties.

\subsection{Characterization techniques}

Although the differences found in optoelectronic properties of perovskite SC presented in Table 2 and Table 3 can be related to difference in crystal quality, one must not underestimate the impact that the crystal morphology, the experimental conditions and the techniques employed have on the measured properties. Here, we briefly summarize the characterization techniques employed to assess optoelectronic properties presented above. The objective of this section is to highlight the existing challenges to properly evaluate MAPX material quality, and provide with a critical view on data interpretation.

Diffusion length The diffusion length is a critical parameter that must be taken into account for an efficient design of a solar cell architecture. It is calculated as:

$$
L_{D}=\sqrt{\frac{k_{B} T}{q} \mu \tau}
$$

where $k_{B}$ is Boltzmann's constant, $\mathrm{T}$ the temperature, $\mathrm{q}$ the electronic charge, $\mu$ the mobility and $\tau$ the lifetime. As a consequence of the vast range of lifetime and mobility values reported for SC, diffusion length values range from $3 \mu \mathrm{m}$ to $3 \mathrm{~mm}(120)$.

Lifetime Carrier lifetime is typically measured by exciting the material with a pulsed laser and observing the characteristic decay profile associated with the recombination of the photo-generated carriers $(47,96,111)$ (Figure 12a, b and c). A short and a long lifetime are commonly extracted and attributed to surface and bulk recombination $(69,71,117,119)$. 
a

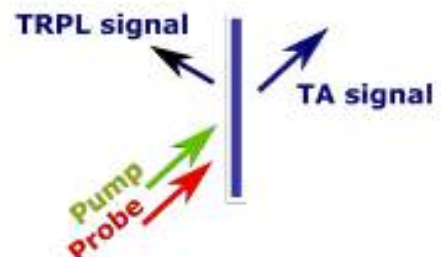

d

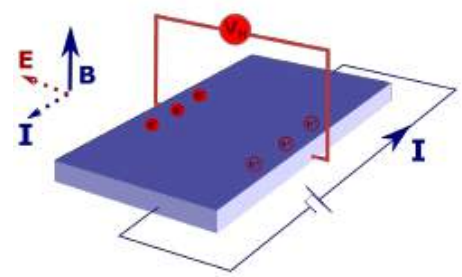

Hall measurement set-up b
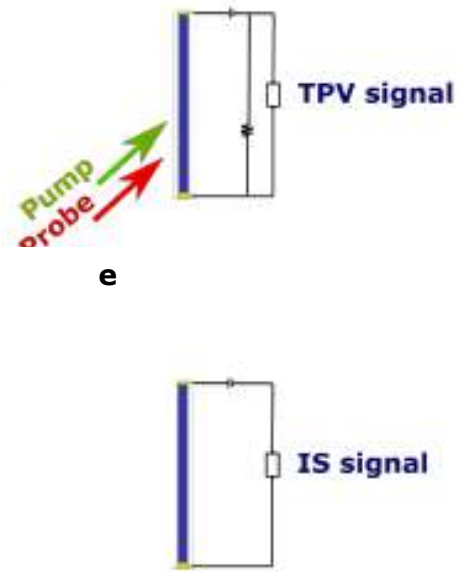
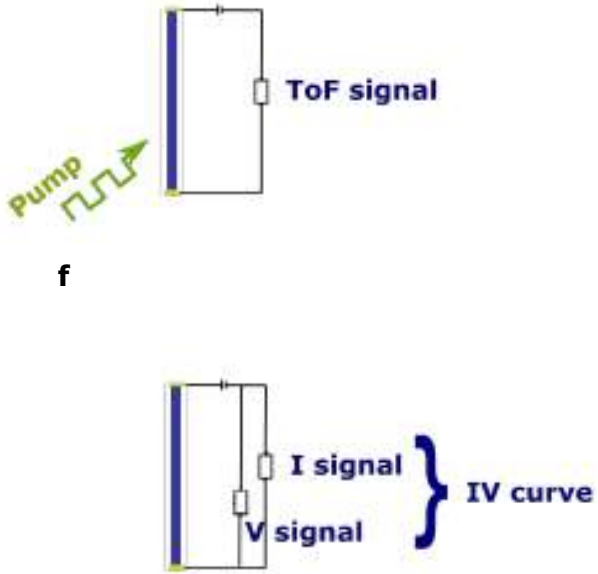

Figure 12 - Diagram of the different techniques commonly used to characterized SC properties. a) Transient absorption (TA) and Time-resolved photoluminescence (TRPL), b) Transient photovoltage (TPV), c) Timeof-flight measurements (ToF), d) Hall measurements, e) Impedance Spectroscopy (IS) and f) I-V measurements.

Time-resolved photoluminescence (TRPL) probes the photoluminescence as a function of time, whereas transient absorption (TA) monitors changes in absorption $\Delta \mathrm{A}$ (associated with a change in charge carrier density in the conduction band upon recombination) (121). Both TPRL and TA techniques allow one to measure lifetime without depositing any contacts on the substrate.

Transient photovoltage (TPV) measures the perturbation to the open circuit voltage, created upon laser excitation. Measurements are performed on a cell connected to an oscilloscope (93).

In time-of-flight (ToF) measurements a short pulsed laser is used to generate photocarriers, at a given repetition rate. Two contacts are deposited on the perovskite material and a transient current (I) is extracted upon charge carrier drifting under DC bias. Among other parameters, the transit time (t), which is the time needed for photo-induced carriers to travel through the crystal thickness, can be extracted and allows one to calculate carrier lifetime (122) using the following relation where $\tau_{h / e}$ is the hole or electron lifetime.

$$
i(t) \propto e^{\frac{-t}{\tau_{h / e}}}
$$

In alternative, lifetime can also be measured using impedance spectroscopy (IS) (Figure 12e). The impedance response of a perovskite based cell to a sinusoidal perturbation is probed at different frequencies under a potential bias. The imaginary part of the impedance (reactance Z") is then plotted as a function of the real part (resistance Z') and fitted with an equivalent circuit model to extract the lifetime $(123,124)$.

Mobility Mobility is typically obtained by Hall effect or space charge limited current (SCLC) measurements. The Hall effect is based on the Lorentz force and is measured under an external magnetic field normal to the sample surface (Figure 12d). Typically four contacts are deposited on the perovskite surface and, as current flows between two opposite contacts, electrons experience a force in a normal direction to both the applied magnetic field and the current direction, which results in the accumulation of negative charges at one side of the sample and positive charges at the opposite side. The resultant potential is then measured between the other two contacts. This Hall voltage allows to determine the density of charge carrier in the perovskite, and along with resistivity measurements mobility can be calculated $(88,117)$.

In alternative, mobility can be extracted from ToF transit time (t) with the following formula where $\mathrm{L}$ is the thickness of the crystal and $\mathrm{U}$ the applied bias.

$$
\mu_{h / e}=\frac{L^{2}}{t U}
$$

Mobility can also be extracted from simple electron-only or hole-only I-V curves (Figure 12f). Such curves typically present an ohmic region $\left(\mathrm{I} \propto \mathrm{V}^{n=1}\right)$, followed by a trap-filled limit region $\left(\mathrm{I} \propto \mathrm{V}^{n>3}\right)$ and a child region $\left(\mathrm{I} \propto \mathrm{V}^{n=2}\right)(56,69,85,89)$. Mobility is calculated by fitting the child region with the Mott-Gurney law, where $\epsilon$ is the permittivity of the material, and d its thickness.

$$
J=\frac{9}{6} \mu \epsilon \frac{V^{2}}{d^{3}}
$$


Trap density The trap density reported in Table 2 and 3 is systematically determined at the trap-filled limit voltage $V_{T F L}$ corresponding to the transition between the ohmic and trapped filled region by the following equation where $\mathrm{q}$ is the electronic charge, $\mathrm{d}$ the thickness of the crystal, $\epsilon$ the dielectric constant and $\epsilon_{0}$ is the vacuum permittivity.

$$
n_{\text {traps }}=\frac{2 \epsilon \epsilon_{0} V_{T F L}}{q d^{2}}
$$

\subsection{Challenges in data interpretation}

The samples are sometimes probed as part of simple devices (IS, ToF, TPV, Hall, SCLC) and sometimes probed as free standing samples (TRPL, TA), as presented above. Therefore, charge transport layers or electrodes used to build those devices can impact charge transport, even at open circuit conditions, thus biasing lifetime, mobility and trap densities measured. This is evidenced by the strong variation $(>10 \mathrm{x})$ in TRPL lifetime as a function of the layer stack, or as a result of which side of the stack is excited (Figure 13b, e and f) (86, 89).

Similarly, I-V curves used to calculate mobility and trap densities are typically obtained from very simple devices such as $\mathrm{Au} / \mathrm{PVK} / \mathrm{Au}$ but they can also be measured from more complex ones (93), which can affect the transport properties and thus the apparent optoelectronic properties, while other techniques used to determine mobility such as Hall and ToF measurements also use contacts and can thus be impacted by the type and quality of those contacts.

Another challenge to compare data obtained with different techniques lie in the fact that some of them expose the perovskite material to an external bias (IS, Hall, SCLC, ToF) while other do not (TPRL, TA, TPV) as summarized in Figure 13a. However, when the cell is exposed to an external bias, ion migration come into play and the accumulation of charged species at contacts might shield the bulk material from the applied voltages (ToF, SCLC) or impact voltage measurements (Hall). This was evidenced by Duijnstee et al., who found one order of magnitude lower conductivity and one order of magnitude higher trap density compared to SCLC measurements, if the sample was probed with short TPV pulses that inhibited ion migration (Figure 13c) (125). Similar conclusions were also drawn by other authors (122).

Calculation of trap densities from optical methods and models allow to discard issues of ion migration due to electrical contacts. Wu et al. calculated trap densities of $5 \cdot 8 \cdot 10^{15} \mathrm{~cm}^{-3}$ for bulk MAPB SC using two photons (2P) absorption, a value far superior to that commonly extracted from I-V measurements (126) (Figure 13d). Nevertheless, the trap density measured with optical methods for MAPB SC was still lower than that of MAPB PC $\left(4 \cdot 10^{15} \mathrm{~cm}^{-3}\right.$ vs $\left.7 \cdot 10^{16} \mathrm{~cm}^{-3}\right)(52)$.

The characterization techniques discussed in this section, probe optoelectronic properties near the Fermi level by injecting (Hall, SCLC) or generating (ToF, TRPL, TA, TPV) free charges in the material. However the probed non-equilibrium Fermi level is not always the same and thus the measurements is not affected by the same trap states (120). For example some measurements are performed in the dark (TRPL, TA, ToF) while other measurements are performed under illumination (TPV, IS), which impact the density of generated carriers. IS lifetime was reported to decrease sharply from dark condition to 0.1 sun and exponentially from 0.1 sun illumination to one sun illumination, as the density of free charge carriers increased (124). Similarly, for TRPL, TA, TPV, as well as ToF the level probed depends on the pulsed laser intensity. 
a

Contactless With contacts

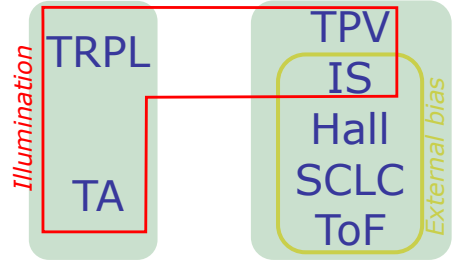

C

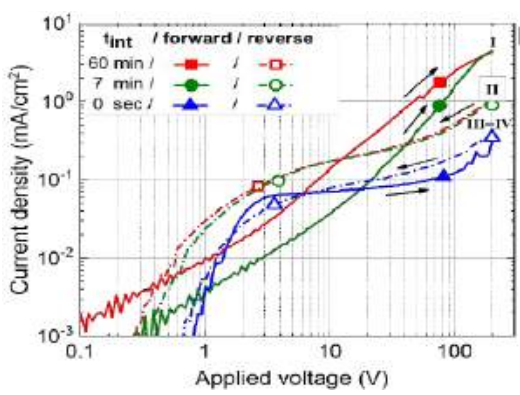

b

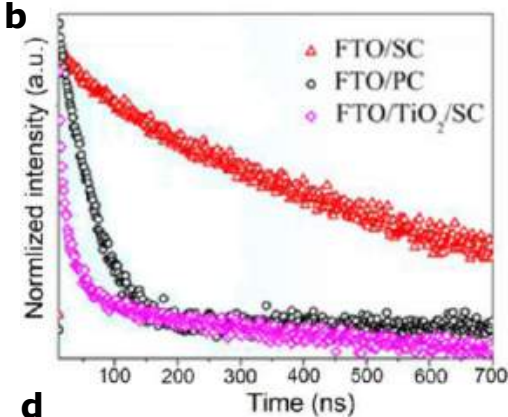

d

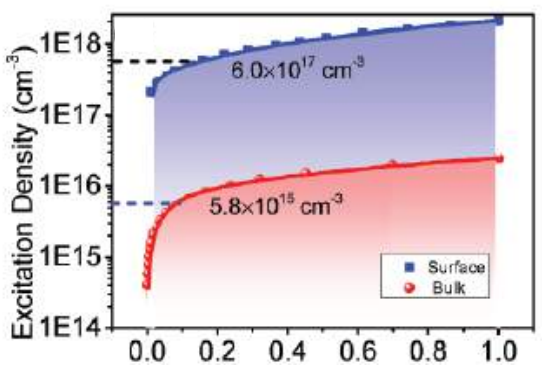

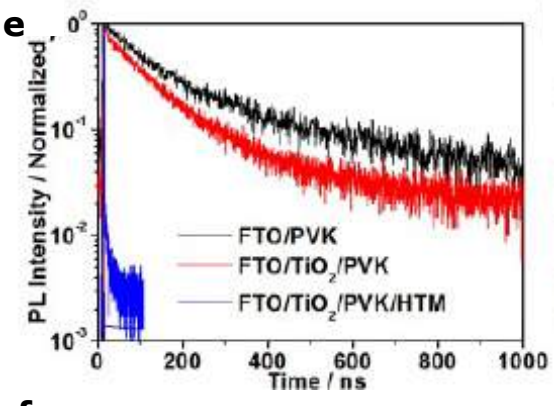

f

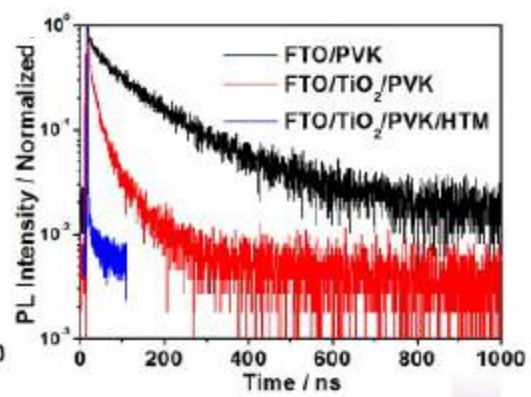

Figure 13 - Impact of characterization techniques and conditions on the measured optoelectronic properties of MAPX-SC. a) Diagram of the different conditions under which MAPX SC are probed b)TRPL of SC and PC MAPI on different substrates (from ref.(86)) c) Large hysteresis between forward and reverse I-V traces when the interval time ( $\mathrm{t}_{\text {int }}$, waiting time between voltage sweeps cycles) is long enough to allow for ionic species to return to their initial positions (red and green lines) and small hysteresis between forward and reverse I-V traces when the species don't have enough time to migrate back to their initial position before the next scan (blue line) (from ref.(125)) d) Carrier-intensity-dependent PL intensities and the fitting (solid lines) that yield the trap density at the surface and in the bulk (from ref.(126)). TRPL of MAPB on different substrates with light on e) MAPB side f) on FTO side (from ref.(89)).

A standardized protocol would be welcomed to being able to compare in a reliable fashion intrinsic material properties, free from experimental bias, especially for trap densities and mobility measurements that are highly impacted by ion migration. Optoelectronic properties of MAPX crystals presented in Table 2 and 3 should be therefore compared with precautions. It is important to note that when probed in the same conditions and with the same techniques MAPX SC always displayed optoelectronic properties superior to MAPX PC (52). As a conclusion, if SC halide perovskite could be integrated in solar cell without degradation in properties, SC solar cell would be expected to work better than their PC counterparts.

\section{Single crystals stability}

Polycrystalline perovskite solar cell have achieved above $25 \%$ efficiency in the past year, making it among the best single junction cells in terms of efficiency. However, the poor stability hinders the commercialization. The stability of SC in ambient conditions was reported to be better than that of PC thanks to the absence of grain boundaries and to the lower amount of crystallographic defects.

It was reported that MAPI single cystals showed no apparent degradation over 6 months (Figure 14c) (65) or even 2-3 years despite the fact that they were stored in ambient atmosphere (19). SC solar cells without encapsulation showed no change in PCE after storage in air for 30 days (MAPI) (Figure 14a) (93) and retained 93\% of their initial efficiency after 1000 hours in air (MAPB) (Figure 14b) (89). Defect density seems to play a role for long term stability, as evidenced by Yao et al. that showed better stability for crystals with lower trap density (grown at low temperature) after 10,000 working cycles in air (Figure 14d) (47). 
a

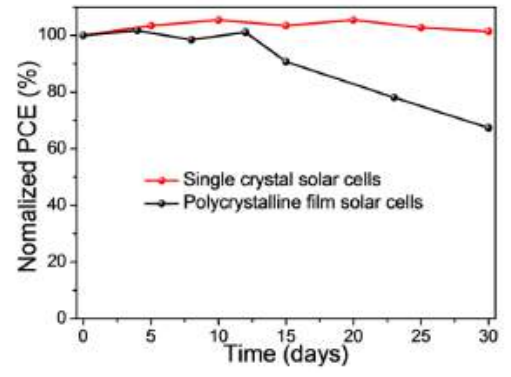

d
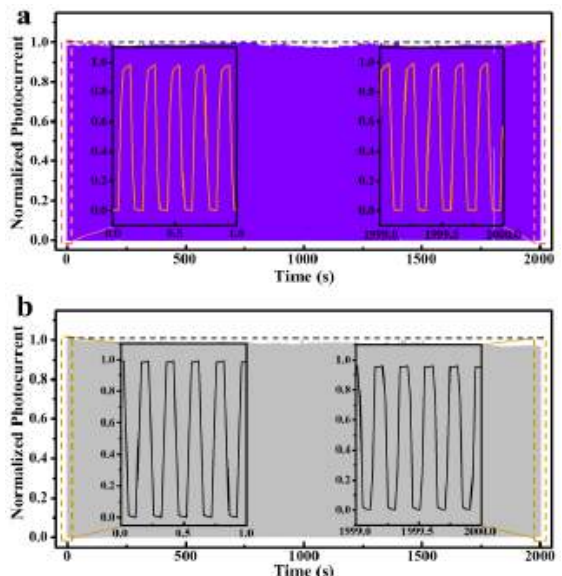

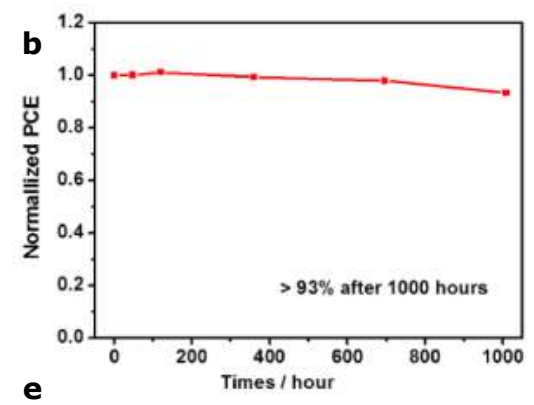

Before water mist erosion After water mist erosion
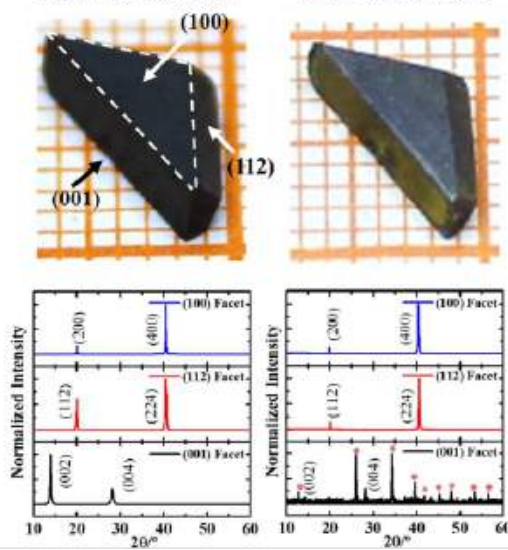

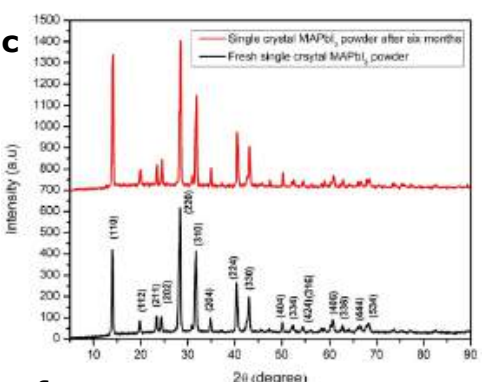

f
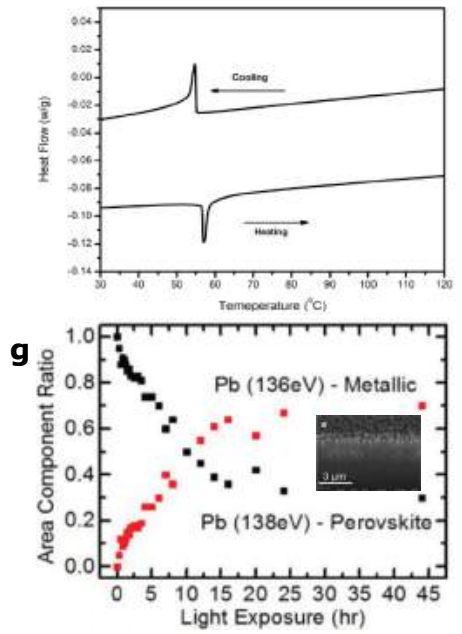

Figure 14 - Stability of MAPX-SC. a) Normalized PCE of the SC solar cells and PC-film solar cells in ambient environment without encapsulation as a function of storage time. The temperature and relative humidity is $23^{\circ}$ and $30 \%$ (from ref (93) (SI)) b) Stability test as a function of storage time in dry air without encapsulation (from ref SI (89) (SI) ) c)Powder X-Ray Diffractograms of MAPI-SC after growth (bottom) and after six months (top) (from ref (65) d) Temporal photoresponse of MAPB-SC grown at low-temperature (LT-MAPB) (top) and high-temperature (HT-MAPB) (bottom) under modulated illumination without encapsulation in air with 10000 working cycles (from ref (47)) e) Optical photographs and and diffractograms of the (100) facet, (112) facet and (001) facet before and after suffering from water mist erosion for $60 \mathrm{~s}$. Asterisks indicate the peaks of $\mathrm{PbI}_{2}$ (from ref (127)) f) DSC thermogram of MAPI-SC (from ref $(65) \mathrm{g})$ The ratio of perovskite $\mathrm{Pb}$ to metallic $\mathrm{Pb}$ with increasing light exposure and $\mathrm{X}$-ray exposure. The FIB milled trench of the light exposed region revealed voids about 1-3 $\mu \mathrm{m}$ deep into the material (from ref (128)).

\subsection{Heat}

SCs are able to withstand higher temperatures. PC perovskite thin films are known to decompose around $150^{\circ} \mathrm{C}$ while TGA thermograms of SCs revealed that they remained stable up to $240^{\circ} \mathrm{C}$ for MAPI $(65,88), 214^{\circ} \mathrm{C}$ for MAPC and $257^{\circ} \mathrm{C}$ for MAPB (36). Prathreek et al. hypothesized that the higher decomposition temperature of MAPI-SC could, in their case, be attributed to the absence of trapped water, as revealed by the absence of endothermic peak at $100^{\circ} \mathrm{C}$ in the DSC thermogram shown if Figure 14f, commonly reported in other studies on PC-films (65).

\subsection{Moisture}

It is widely recognized that in PC films, moisture degradation originates at grain boundaries, making SC less sensitive to moisture. Wang et al. studied the impact of moisture on a freshly cleaved MAPB single crystal in a UHV chamber at a humidity level of $100 \%$. They concluded that degradation occurred at a moisture level of $10^{8}$ Langmuir and was limited to the top $0.37 \mathrm{~nm}$ surface (129). The anisotropy of moisture-induced erosion in MAPI was studied by exposing to moisture a SC exhibiting (001), (100) and (112) facets. XRD measurements provided evidence that the (001) facet had undergone faster degradation into $\mathrm{PbI}_{2}$ (Figure 14g) (127).

\subsection{Light}

Light has been reported to damage the surface of perovskite by promoting ion migration (130). The activation energy for ion migration in SCs was reported to be reduced to $0.33 \mathrm{eV}$ under illumination from the original $0.83 \mathrm{eV}$ measured in dark conditions (15). Ecker et al. studied the stability of a MAPB single crystal exposed to strong light illumination $(\approx 7$ sun) in a UHV chamber. X-ray photoemission spectroscopy measurements 
revealed that, within 10 hours, half of the $\mathrm{Pb}^{2+}$ at the surface had been converted into metallic $\mathrm{Pb}$, whereas $\mathrm{C}$, $\mathrm{N}$ and Br elemental concentration dropped significantly; this resulted in the creation of $\mu \mathrm{m}$ sized voids at the crystal surface, as evidenced by FIB images (Figure 14)g) (128). TEM results further showed that single crystals broke apart into nanocrystals upon light exposure, in accordance to previous studies reported the formation of grain boundaries upon aging $(131,132)$. MAPI SC were found to degrade under UV-light even at temperature as low as $5 \mathrm{~K}$ and in a similar fashion to PC perovskite (133).

In conclusion, SC are more stable than their PC counterparts, but intrinsically sensitive to light (128, $133)$ and moisture $(129,131)$ affecting the surface of SC, that have been reported to restructure to a PC-like surface (132), which inevitably impacts carrier transport. Potential approaches to mitigate this problem will be discussed in sections 8.3 and 8.1 .

\section{State of the art MAPX SC solar cell devices}

The following section reviews different strategies to integrate thin film MAPX SC into vertical and lateral solar cells architecture, and compare device performance.

\subsection{Integration in vertical cells}

MAPX thin films have been successfully integrated in vertical cells with the standard (electrons collected on the illuminated side) as well as the inverted configuration (electrons collected at the back) (Figure 15a).

\subsubsection{Charge transport layers (CTL) and electrodes}

In standard cells, $\mathrm{TiO}_{2}$ is typically used as electron transport layer (ETL) and FTO as top electrode (86, 89, 97, 114). Spiro-OMeTAD is used as hole transport layer (HTL) and Au as back electrode.

In inverted cells, ITO or FTO are used as top electrodes (93). Poly(triaryl amine) (PTAA) is used as a HTL $(27,93,134)$, so as PEDOT $(92,105)$ and spiro-OMeTAD (6). For the ETL, PCBM is largely used (92, 105), sometimes with the interfacial layer LiF (6) or a C60-Bathocuproine (BCP) buffer layer (93) between the ETL and the electrode, that can also be also used without any ETL $(27,134)$.

CTL are commonly deposited in the liquid form by spin coating on the electrode material or by spray coating on the perovskite layer to avoid using solvents that might deteriorate MAPX surface $(6,89,105,115)$. Thermal evaporation of $\mathrm{LiF}, \mathrm{C} 60$ and $\mathrm{BCP}(27)$, thermal or e-beam evaporation of electrodes (Au, Ag, Al), melting of Ga, magnetron sputtering (ITO, Au grids) proved compatible with MAPX SC, enabling to obtain complete solar cell devices $(5,6)$. 
a

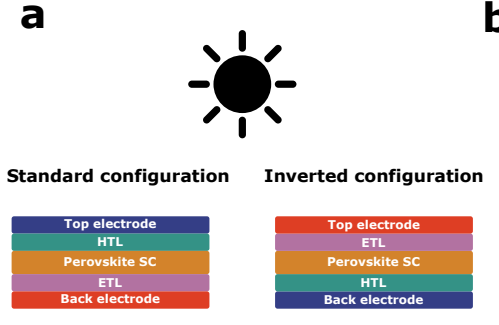

d

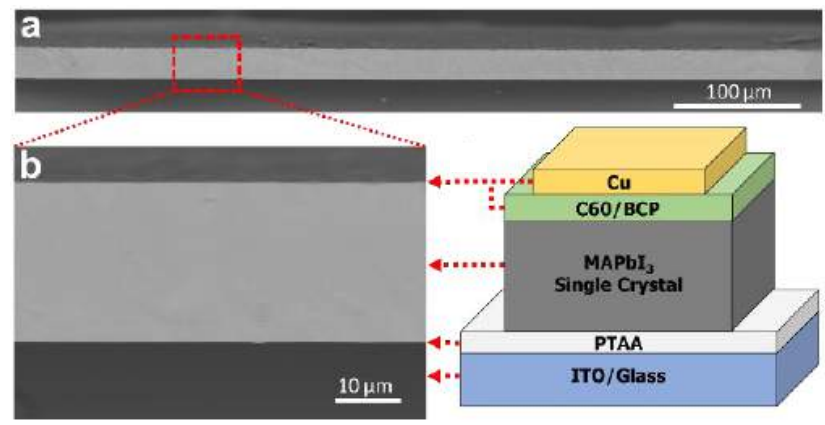

f

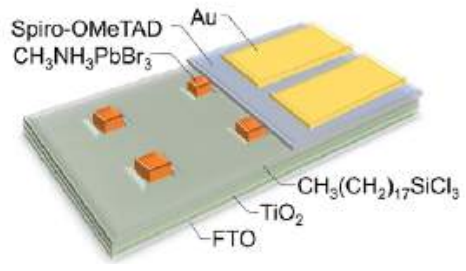

b

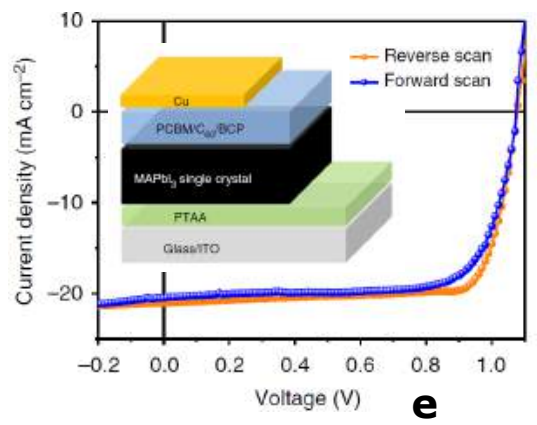

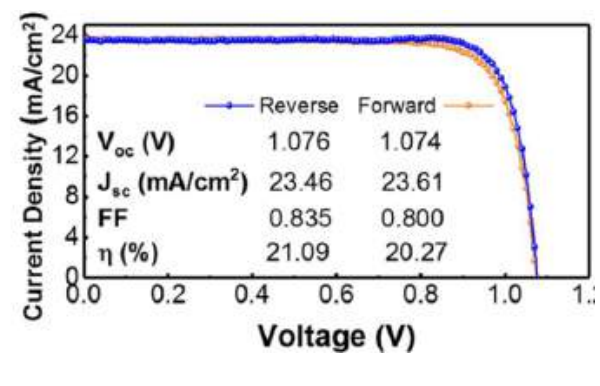

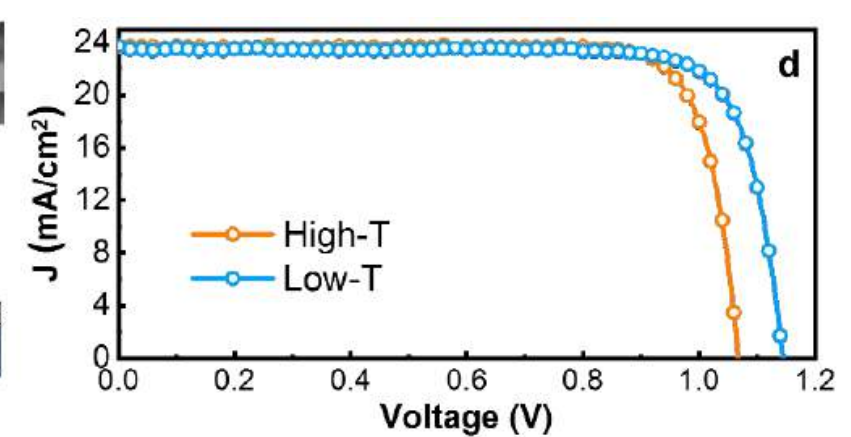

g
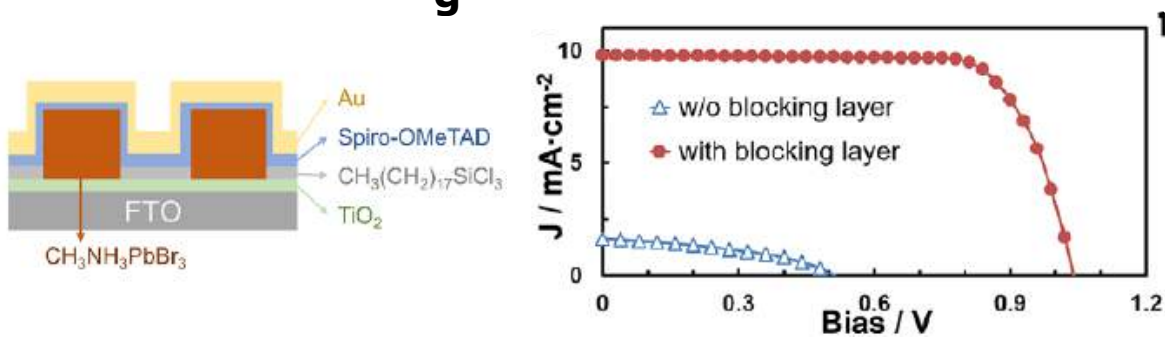

Figure 15 - Integration in vertical cells. a) Diagram of normal and inverted configuration for vertical cells; b) Device structure and $\mathrm{J}-\mathrm{V}$ characteristics of the SC solar cell after MAI treatment (from ref (93)) c) J-V curves in forward-(orange) and reverse-scan (blue) mode and the corresponding photovoltaic parameters under 1 sun illumination for an improved cell fabricated and tested in nitrogen (from ref.(134)); d) Device structure and cross-sectional SEM images of Alsalloum et al. record-cell and e) J-V curves showing the best performances of SC solar cells made from low-temperature (low-T) grown crystal crystallization (from ref.(27)) f) Schematic of the solar cell based on patterned MAPB SC microarray and g) current density-voltage characterization of the devices with and w/o the blocking layer under 1 sun illumination (from ref.(114)).

\subsubsection{Thin films}

On chip synthesis enables for swift integration in a device and a better contact between the electrodes and the absorber layer. The film is grown between two substrates (via the SLG technique), at least one of it being an electrode (possibly coated with a CTL). One of the substrate is then peeled of, leaving a half cell ready to be completed $(27,86,89,93,96,134)$.

Chen et al. grew a MAPI thin film directly on glass FTO coated with PTAA (HTL). They then passivated the perovskite surface by spin coating a MAI solution before completion of the cell with an ETL (PCBM/C60/BCP) and a $\mathrm{Cu}$ electrode (Figure 15b), which allowed a 2-5 fold reduction in the trap density and resulted in a $\mathrm{PCE}$ of $17.8 \%$ for the best devices (93).

A PCE exceeding 21\% was eventually achieved in 2019 (Figure 15c) by replacing FTO by ITO and most of all, by fabricating, storing and testing the cell in a nitrogen filled environment. It is worth noticing that when the testing was done in air, a drastic decrease in performances was observed (134).

A record of $21.9 \%$ was set by Alsalloum et al. in 2020 with the same configuration (Figure $15 \mathrm{~d}$ and e). The authors conducted the growth at a lower temperature (maximum temperature of $90^{\circ} \mathrm{C}$ ) thanks to the addition of an anti-solvent to the ITC precursor solution which i) yielded to SC thin films of better quality (average lifetime $164 \mathrm{~ns}$ compared for $42 \mathrm{~ns}$ for films grown at a maximum temperature of $130{ }^{\circ} \mathrm{C}$ as well as a 3 -fold increase in PL) ii) prevented the PTAA layer from dissolving in the solution (27)).

Notably in 2020 Kong et al. succeeded in growing SC-MAPI by a modified AVC method (section 2.1.1, Figure $5 \mathrm{~b}$ ) and to integrate it in a solar cell. A thin film of only $300 \mathrm{~nm}$ was grown directly on ITO/PEDOT:PSS and a $\mathrm{PC}_{61} \mathrm{BM} / \mathrm{BCP}$ was deposited afterward in nitrogen filled environment. The device yielded to a $\mathrm{PCE}$ of $20.1 \%$, higher than the PCE of the control device made out of PC MAPI. 
A lamination process can be used as an alternative to transfer the perovskite material onto a new substrate containing already electrode and CTL. The addition of the other CTL/electrode stack enable for completing the cell. The lamination process can however impede the formation of good contacts at interfaces therefore limiting efficiency (92).

\subsubsection{Patterned thin films}

Fabrication of a half cell using perovskite patterned thin films adds a greater level of complexity as one has to be able to pattern the perovskite film directly on an electrode material.

As an example, the Ostwald ripening assisted photolithography (ORAP, section 2.3.1) process allow one to fabricate patterned perovskite SC microarrays by using difference in wettability of a patterned substrate to split the precursor perovskite solution. To allow for on chip synthesis, an hydrophobic film (polyTPD) was deposited on FTO coated with $\mathrm{TiO}_{2}$ and patterned via photolithography. After phtoresist removal and completion of the cell with a blocking layer $\left(\mathrm{CH}_{3}\left(\mathrm{CH}_{2}\right)_{1} 7 \mathrm{SiCL}_{3}\right)$, a spiro-OMeTAD based HTL and Au electrode, the solar cell achieved a PCE of $7.84 \%$ (Figure $15 f$ and g) (114).

\subsection{Integration in lateral cells}

Lateral cells are built by depositing contacts on the same side of an absorber layer as illustrated in Figure 16a. Perovskite SC, thanks to their long diffusion length and long carrier lifetime, are ideal candidates for lateral cells because spacing between the electrodes can be much superior compared to that of lateral cells built on PC perovskite, thus reducing shadowing effects. In this configuration light absorption and carrier extraction are orthogonalized, since holes and electrons are collected on the sides of the cell as opposed to the top and the back. As a consequence, the thickness of the material can be superior to that of thin films, and lateral cells can even be built on SC surfaces.

a

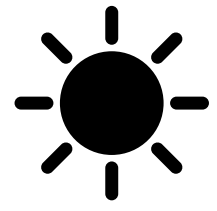

Lateral configuration

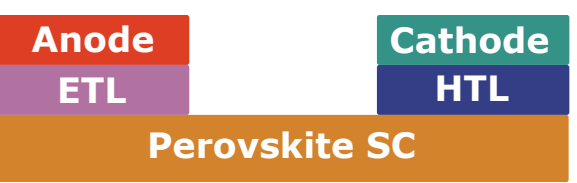

e

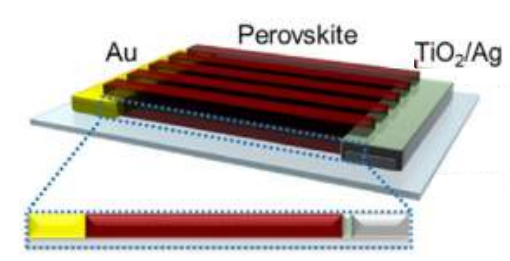

Glass b

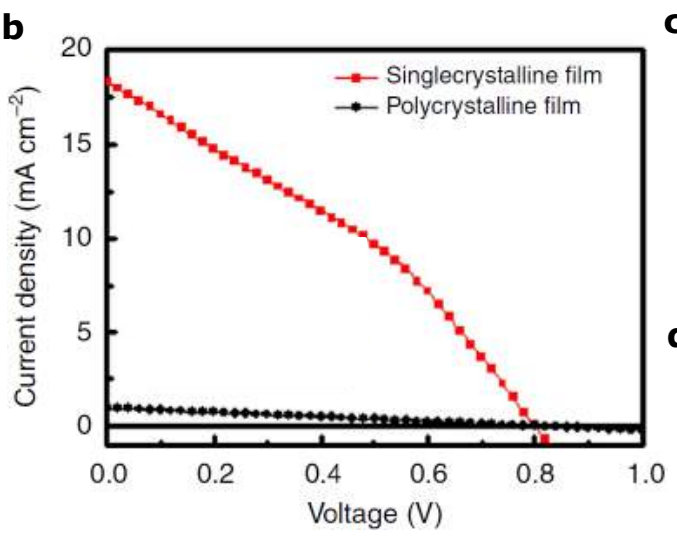

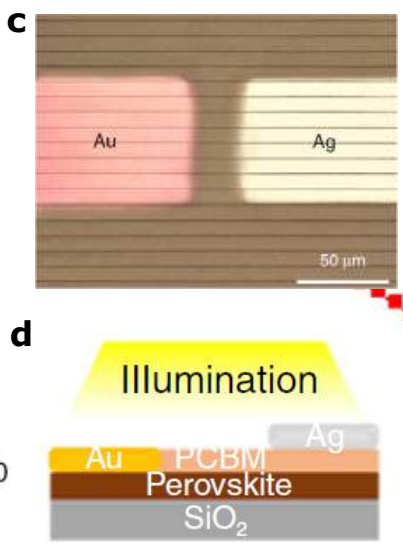

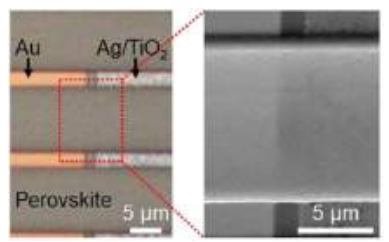

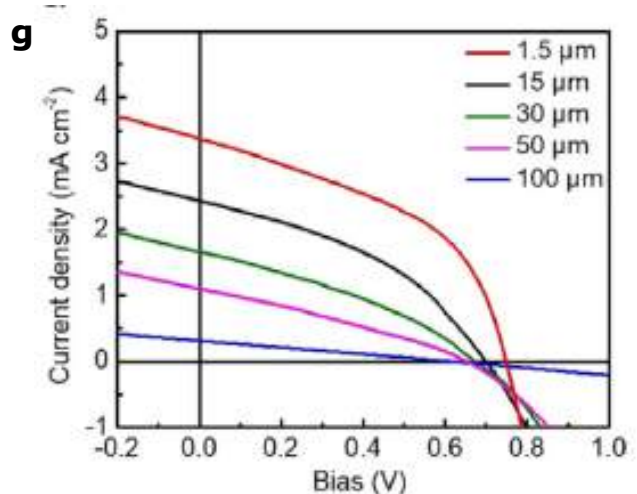

Figure 16 - Integration in lateral cells. a) Diagram of lateral configuration b) J-V curves of Lee et al. SC (red) and PC (black) MAPI thin film lateral solar cells and Schematic device structure of a lateral perovskite solar cell and the direction of illumination (inset) c) An optical microscopic image of a SC-MAPI lateral perovskite solar cell with metal electrodes. The light brown and dark brown lines indicate MAPI-SC and spaces, respectively and d) schematic representation of the device structure (from ref.(115)) e) Schematic illustration of Park et al. device. f) Optical microscope picture and high-resolution SEM image of the single patterned film g) J-V characteristics of lateral single-crystal perovskite solar cells with different gap spacings (from ref.(135)).

In order to extract electrons on one side and holes at the other side of the crystal, either selective contacts are used or a p-i-n junction is created within the crystal. Electrical poling allows to self-dope the crystal through 
ion drift under an external potential field. As the ion migrate one side of the film becomes n-doped while the other becomes p-doped (136). This allowed Dong et al. to fabricate the simplest lateral cell by depositing two parallel Au electrodes on one facet of MAPI bulk crystal and inducing a p-i-n junction by electrical poling between $50 \mathrm{~V}$ and $200 \mathrm{~V}$ (137). Lee et al. combined electrical poling with selective contacts, they deposited an Ag electrode on top of a PCBM layer $33 \mu \mathrm{m}$ appart from an Au electrode and further applied a $13 \mathrm{~V}$ potential to induce the formation of a p-i-n structure within the MAPI crystal and achieved a PCE of 4.83\% (Figure 16b) (115). Both group compared their SC lateral cell to that of a PC cell of the same structure and observed a 44-fold and a 25-fold enhancement for the SC cell respectively.

Perovskite can also be integrated in lateral cell via on-chip synthesis. Park et al. grew arrays of perovskite $\mathrm{SC}$ on a glass substrate on which had been deposited two electrodes. The resultant $\mathrm{Au} / \mathrm{MAPI} / \mathrm{TiO} 2 / \mathrm{Ag}$ cell was further treated with electrical poling to form a p-i-n junction (Figure 16e). By optimizing the electrode spacing to $1.5 \mu \mathrm{m}$ (as shown in Figure 16g) the authors obtained a record efficiency for lateral perovskite cell of $9.5 \%$ under 0.1 sun illumination and $7.43 \%$ under 1.0 sun illumination (135). By taking advantage of the array configuration, they were able to easily create interdigitated units (135).

However, electrical poling was reported to damage the quality of the crystal as well as to induce the formation of grain boundaries (137), questioning its advantages. Liu et al. for example achieved a PCE of $5.9 \%$ with a $\mathrm{Au} / \mathrm{MAPbI} / \mathrm{C} 60 / \mathrm{BCP} / \mathrm{Au}$ configuration (without poling) (138) and Song et al. used the same configuration (Figure 16h) but added a passivation step by spin coating a MAI solution on the MAPI surface which allowed to improve the PCE from $4.04 \%$ to $11.52 \%$ (as shown in Figure 27 a and b) making it the record lateral cell for perovskite SC (139). The same team also investigated larger area devices by implementing interdigitated electrodes. Devices 36 times larger were fabricated and exhibited a PCE of $6.30 \%$ (139).

Integration in lateral cells could benefit from the advanced architectures developed in nanoscale solar cells, such as core-shell nanowires in a vertical or horizontal configuration, to take advantage of orthogonalization of carrier and photon transport (109, 140-142).

The devices performance obtained for both vertical and lateral cells are summarized in Table 4 and Figure 17. 


\subsection{Device performances}

Vertical structure perovskite thin film solar cell

\begin{tabular}{|c|c|c|c|c|c|c|c|c|}
\hline Integration & $\begin{array}{l}\text { Thickness } \\
\qquad \mu \mathrm{m}\end{array}$ & Treatment & $\begin{array}{l}\text { Device } \\
\text { geometry }\end{array}$ & PCE & $\begin{array}{c}\mathrm{J}_{S C} \\
\mathrm{~mA} \cdot \mathrm{cm}^{-2}\end{array}$ & $\begin{array}{c}\mathrm{V}_{O C} \\
\mathrm{~V}\end{array}$ & $\begin{array}{c}\mathrm{FF} \\
\%\end{array}$ & Ref \\
\hline & 0.3 & Cleaving & $\begin{array}{c}\text { ITO/PEDOT:PSS/MAPI } \\
\text { PCBM/Al }\end{array}$ & 0.01 & 0.04 & 0.55 & 35 & $(105)$ \\
\hline Bulk & $>190$ & Slicing & $\begin{array}{c}\mathrm{Au} / \mathrm{ITO} / \mathrm{SpiroMeoTAD} / \mathrm{MAPI} / \\
\mathrm{PCBM} / \mathrm{LiF} / \mathrm{Ag} / \mathrm{Au}\end{array}$ & 4 & 13 & 1 & 30 & (6) \\
\hline $\begin{array}{l}\text { Lamination } \\
\text { SLG }\end{array}$ & 50 & $\mathrm{X}$ & $\begin{array}{l}\mathrm{Ag} / \mathrm{PCBM} / \mathrm{MAPI} / \\
\mathrm{PEDOT}: \mathrm{PSS} / \mathrm{ITO}\end{array}$ & 4 & 22 & 0.75 & 27 & $(92)$ \\
\hline \multirow{7}{*}{$\begin{array}{l}\text { On chip } \\
\text { SLG }\end{array}$} & 15 & $\mathrm{X}$ & $\begin{array}{l}\mathrm{Au} / \mathrm{MAPB} / \\
\mathrm{ITO}\end{array}$ & 2.3 & - & - & - & $(96)$ \\
\hline & 16 & $\mathrm{X}$ & $\begin{array}{c}\text { FTO/TiO } / \text { MAPB/ } \\
\text { SSpiroMeoTAD-Li-TFSi/Au }\end{array}$ & 7.11 & 8.77 & 1.31 & 62 & $(89)$ \\
\hline & $50-200$ & $\mathrm{X}$ & $\begin{array}{c}\mathrm{FTO} / \mathrm{TiO}_{2} / \mathrm{MAPI} / \\
\text { SpiroMeoTAD/Ag }\end{array}$ & 8.78 & 22.28 & 0.67 & 59 & $(86)$ \\
\hline & 10 & $\begin{array}{c}\text { Surface } \\
\text { passivation }\end{array}$ & $\begin{array}{l}\mathrm{FTO} / \mathrm{PTAA} / \mathrm{MAPI} / \\
\mathrm{PCBM} / \mathrm{C} 60 / \mathrm{BCP} / \mathrm{Cu}\end{array}$ & 17.8 & 21 & 1.08 & 79 & \multirow[t]{2}{*}{ (93) } \\
\hline & 0.3 & $\mathrm{X}$ & 20.1 & 22.6 & 1.08 & 82.5 & $(95)$ & \\
\hline & 20 & $\mathrm{X}$ & $\begin{array}{l}\text { ITO/PTAA/MAPI/ } \\
\text { C } 60 / \mathrm{BCP} / \mathrm{Cu}\end{array}$ & 21.1 & 23.46 & 1.08 & 83 & $(134)$ \\
\hline & 20 & $\mathrm{X}$ & $\begin{array}{l}\text { ITO/PTAA/MAPI/ } \\
\mathrm{C} 60 / \mathrm{BCP} / \mathrm{Cu}\end{array}$ & 21.9 & 23.68 & 1.14 & 81 & $(27)$ \\
\hline $\begin{array}{l}\text { On chip } \\
\text { CTAC }\end{array}$ & 3 & $\mathrm{X}$ & $\begin{array}{c}\mathrm{FTO} / \mathrm{TiO}_{2} / \mathrm{MAPB} / \\
\mathrm{Au}\end{array}$ & 6.53 & 9.96 & 1.36 & 69 & $(97)$ \\
\hline $\begin{array}{l}\text { On chip } \\
\text { ORAP }\end{array}$ & 3 & $\mathrm{X}$ & $\begin{array}{c}\mathrm{FTO} / \mathrm{TiO}_{2} / \mathrm{MAPB} / \\
\mathrm{BL}^{1} / \mathrm{SpiroOMeTAD} / \mathrm{Au}\end{array}$ & 7.84 & 9.79 & 1.01 & 77 & (114) \\
\hline
\end{tabular}

Lateral structure perovskite solar cell

\begin{tabular}{|c|c|c|c|c|c|c|c|c|}
\hline Integration & $\begin{array}{c}\text { Electrode } \\
\text { spacing }(\mu \mathrm{m})\end{array}$ & Treatment & $\begin{array}{c}\text { Device } \\
\text { geometry }\end{array}$ & PCE & $\begin{array}{c}\mathrm{J}_{S C} \\
\mathrm{~mA} \cdot \mathrm{cm}^{-2}\end{array}$ & $\begin{array}{c}\mathrm{V}_{O C} \\
\mathrm{~V}\end{array}$ & $\begin{array}{c}\mathrm{FF} \\
\%\end{array}$ & Ref \\
\hline Bulk & 70 & $\begin{array}{l}\text { Electrical } \\
\text { poling }\end{array}$ & $\begin{array}{c}\mathrm{Au} / \mathrm{MAPI} / \\
\mathrm{Au}\end{array}$ & 1.88 & - & - & - & (137) \\
\hline \multirow{3}{*}{ SLG } & 33 & $\begin{array}{l}\text { Electrical } \\
\text { poling }\end{array}$ & $\begin{array}{c}\mathrm{Au} / \mathrm{PCBM} / \mathrm{MAPI} / \\
\mathrm{Ag}\end{array}$ & 4.83 & 18.33 & 0.8 & 33 & (115) \\
\hline & 1.5 & $\begin{array}{l}\text { Electrical } \\
\text { poling }\end{array}$ & $\begin{array}{c}\mathrm{Au} / \mathrm{MAPI} / \\
\mathrm{TiO}_{2} / \mathrm{Ag}\end{array}$ & 7.43 & 19.60 & 0.77 & 49 & (135) \\
\hline & 50 & $\begin{array}{c}\text { Surface } \\
\text { passivation }\end{array}$ & $\begin{array}{c}\mathrm{Au} / \mathrm{MAPI} / \\
\mathrm{C} 60 / \mathrm{BCP} / \mathrm{Au}\end{array}$ & 11.52 & 22.49 & 0.93 & 55 & (139) \\
\hline $\begin{array}{c}\text { Free } \\
\text { standing }\end{array}$ & 50 & $\mathrm{X}$ & $\begin{array}{c}\mathrm{Au} / \mathrm{MAPI} / \\
\mathrm{C} 60 / \mathrm{BCP} / \mathrm{Au}\end{array}$ & 5.9 & 5.06 & 0.66 & 44 & (138) \\
\hline
\end{tabular}

Table 4 - Comprehensive summary of SC MAPX solar cells performance

\footnotetext{
${ }^{1}$ Blocking Layer
} 
a

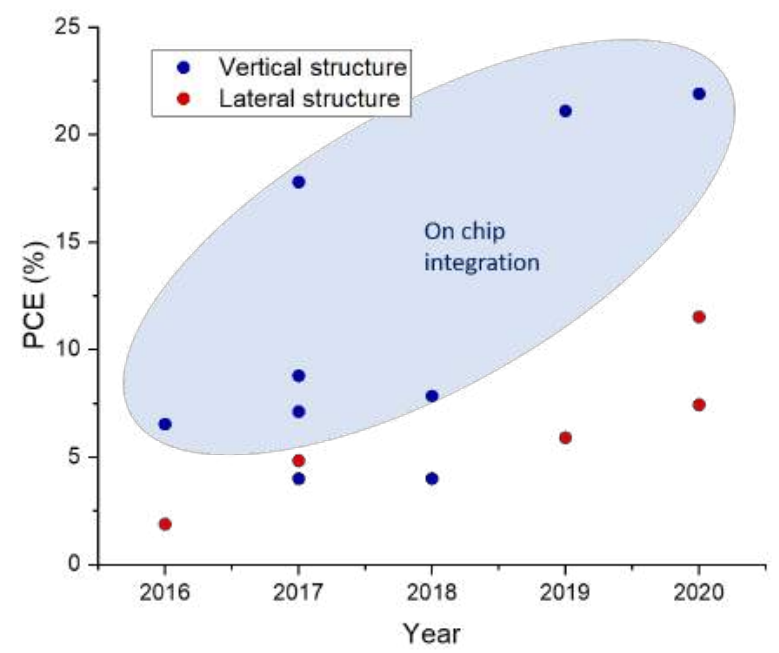

b

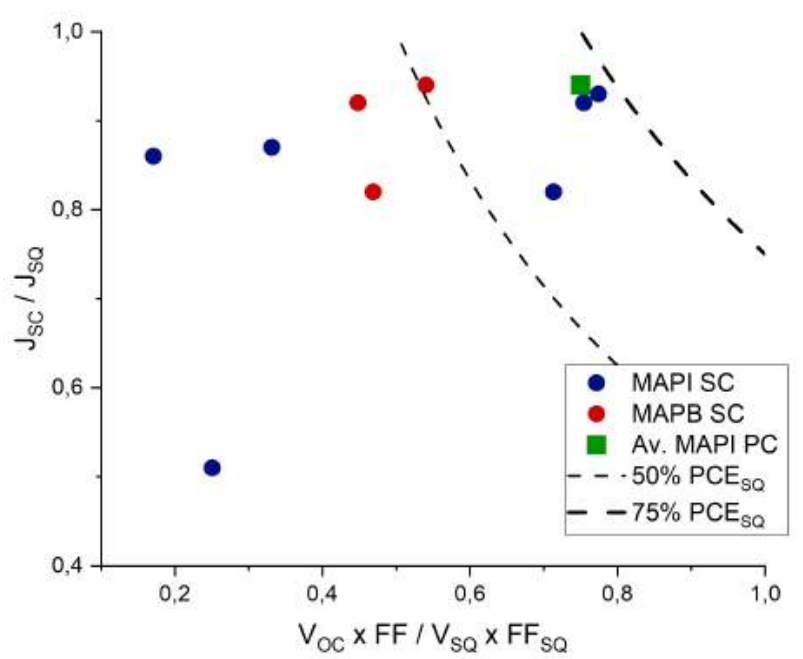

Figure 17 - Performances of SC solar cells. a) PCE as a function of year for SC solar cells (vertical and lateral) b) Comparison between SC and PC perovskite solar cells performances. The short-current and product of open-circuit voltage and fill factor are plotted relatively to the Shockley-Queisser limit (maximum values) which helps understanding whether the overall efficiency lost is due to poor light management ( low $\mathrm{J}_{S C} / \mathrm{J}_{S Q}$ ) or charge carrier management (low $\left.\mathrm{V}_{O C} \mathrm{FF} / \mathrm{V}_{S Q} \mathrm{FF}_{S Q}\right)(143)$.

SC MAPX solar cell is an emerging field with a first report of PCE $>5 \%$ in 2016 (97) (Figure 17a). In only 4 years, and with a limited amount of researchers dedicated to this field compared to that of PC MAPX, it has been possible to grow high quality thin films and to successively integrate them in solar cells. The best SC solar cells reach the performance of average PC solar cells, despite they were foreseen to overtake easily their PC counterparts due to the superior properties of the active layer. This is shown in Figure 17b) that show how best cells perform compared to the SQ limit. The data in the figure suggest that, unlike expectations, there is largely room for improving carriers management in $\mathrm{SC}$ devices, as indicated by the values reached for $\mathrm{V}_{O C} \mathrm{x}$ $\mathrm{FF} / \mathrm{V}_{S Q} \times \mathrm{FF}_{S Q}$.

\section{Why aren't single crystals overtaking poly- crystalline solar cells ?}

Despite the advances in the field of perovskite SC solar cells and the superior properties of the active layer, the PCE of those cells hardly compete with those of their PC counter part (Figure 17b). As discussed earlier, those active layers are integrated as much thicker films, often thicker than the material diffusion length. However, that alone is not able to account for the discrepancy between the expected and experimental performances. In this section, we will try to present critical arguments to shine light on this matter.

A legitimate question would be whether more focus should be put on improving bulk or surface properties, or device architecture to achieve ultimate performance. When comparing optoelectronic properties such as lifetime (see Tables 2,3) it appears that on average bulk SC performs better than SC thin films, suggesting that surface recombination may indeed play a significant role. As a matter of fact, it was shown that in PC perovskites, grain boundaries affected optoelectronic properties up to grain size of $20 \mu \mathrm{m}(12)$, while surface recombination played a dominant in films with larger grains, consistently with theoretical predictions (144). Further evidence is given by the difference between bulk and surface lifetime of PC perovskite as well as the impressive enhancement of PC films properties after surface passivation (145).

One $(1 \mathrm{P})$ and two-photon $(2 \mathrm{P})$ excitation measurements have been used to assess the individual contribution of bulk and surface to the lifetime of perovskite SC (Figure 18) (52, 116, 126, 132, 146). The $100 \mathrm{~nm}$ top surface can be probed with $1 \mathrm{P}$ excitation due to the high absorption coefficient, whereas $2 \mathrm{P}$ excitation allows one to probe material properties $100 \mu \mathrm{m}$ down the surface (126). 
a
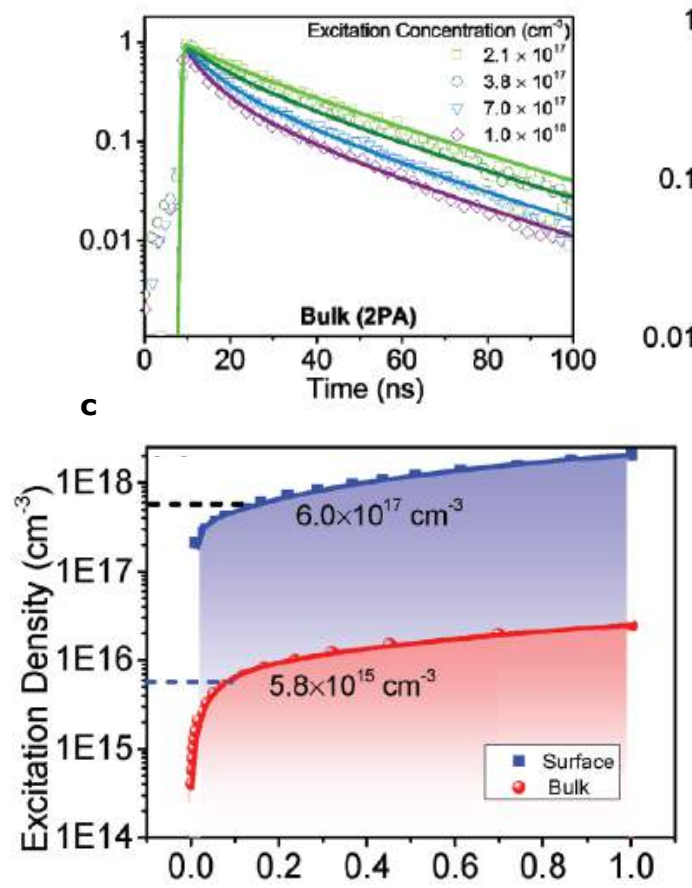

b

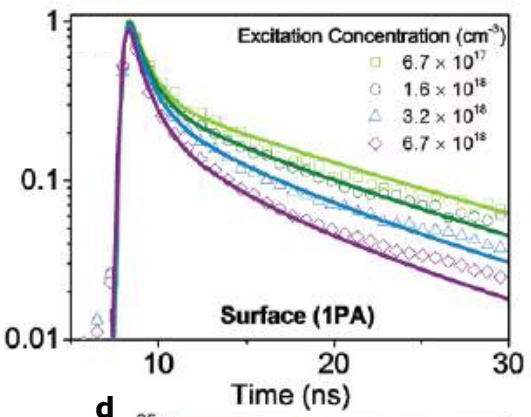

d

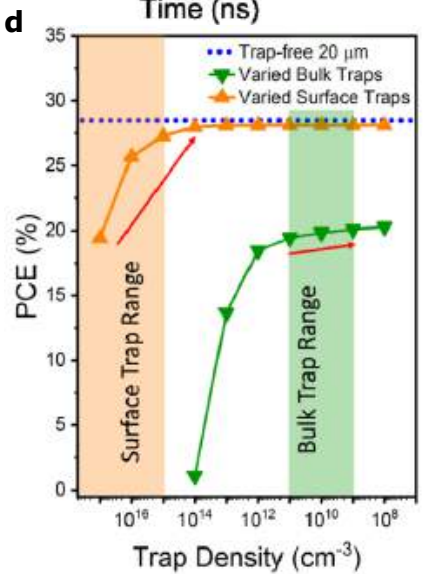

Figure 18 - Surface properties of SC MAPX. TRPL of the MAPB-SC with a) $800 \mathrm{~nm}$ and b) $400 \mathrm{~nm}$ excitations. Solid lines are the fitted curves. c) Surface and bulk properties of MAPB SC. Carrier-intensity-dependent PL intensities and the fitting (solid lines) that yield the trap density at the surface and in the bulk. (From ref.(126)) d) Summary of the effect of bulk and surface trap densities on device efficiency with in green surface trap density held constant at $10^{17}$ $\mathrm{cm}^{-3}$ and variation in bulk trap density; and in orange bulk trap density held constant at $10^{11} \mathrm{~cm}^{-3}$ and variation in surface trap density. The orange region is the reported range for MAPI surface defect densities, and the green region is the reported range for MAPI SC defect densities (From ref.(26)).

Using this techniques bulk and surface lifetimes have been reported for both MAPB and MAPI SC and are summarized in Table 5. In all cases the lifetime is more than one order of magnitude larger in the bulk compared to the surface, providing evidence of significant surface recombination.

\begin{tabular}{cccc}
\hline \hline Material & Bulk Lifetime & Surface Lifetime & Ref \\
\hline MAPB & $1.68 \mu \mathrm{s}$ & $34.88 \mathrm{~ns}$ & $(132)$ \\
MAPB & $4.5 \mu \mathrm{s}$ & $22 \mathrm{~ns}$ & $(116)$ \\
MAPB & $1754 \mathrm{~ns}$ & $60.1 \mathrm{~ns}$ & $(146)$ \\
MAPB & $34 \mathrm{~ns}$ & $1 \mathrm{~ns}$ & $(126)$ \\
MAPI & $109 \mathrm{~ns}$ & $4.9 \mathrm{~ns}$ & $(126)$ \\
\hline
\end{tabular}

Table 5 - Surface and bulk lifetime of perovskite single crystals measured by 1 and 2 photons PL

Based on the measured PL lifetimes, surface and bulk recombination velocities can be calculated. A surface recombination velocity (SRV) of $3.6 .10^{3} \mathrm{~cm}^{-1}$ and $6.7 \cdot 10^{3} \mathrm{~cm} \cdot \mathrm{s}^{-1}$ were reported for MAPI and MAPB single crystals respectively (126). In addition, the surface trap density was estimated to be around $6.0 \cdot 10^{17} \mathrm{~cm}^{-3}$, two orders of magnitude larger that the bulk trap density (126). Bulk and surface diffusion length were also determined with the help of $\mathrm{PC}_{61} \mathrm{BM}$ and Spiro-OMeTAD quenchers deposited on MAPC-SC surface, and revealed that the bulk diffusion length $(2.6-4.3 \mu \mathrm{m})$ was considerably reduced at the surface to $130-160 \mathrm{~nm}$ (126), further confirming the large amounts of traps present on the SC surface. Compared to MAPI PC, the SRV of MAPI SC was measured to be 6 times higher. A possible explanation for this unexpected result could lie in the different surface termination, that is $\mathrm{Pb}^{2+}$-rich in $\mathrm{SC}$, while $\mathrm{C}$ and $\mathrm{N}$ rich in $\mathrm{PC}$, probably resulting from $\mathrm{MA}^{+}$termination (147). $\mathrm{Pb}^{2+}$ clusters, resulting from the high solubility of MAI in solution, have been reported to act as charge traps $(5,29)$.

In addition, by looking at the photovoltaic performance of MAPI SC as a function of bulk defect and surface defect density, the surface of SC perovskite was indeed identified as the major performance bottleneck (Figure 18d) (26). Therefore reducing surface defects of SC perovskite is key to improving device performances. 
Overall, single crystals are challenging to obtain in the form of thin films and to integrate in devices. While progress has been made in recent years, it is still difficult to synthesize sub-micron thick films. In addition, without passivation treatments the excellent properties of SC are masked by poor surfaces properties. This raises doubts on the benefits of perovskite SC compared to PC, that are easier to fabricate. In addition, the issue of $\mathrm{I}_{3}^{-}$traps in MAPI SC, along with issues related to growth temperature, solvent contamination, unstable growth rate and MAPI phase transition have been only recently identified and addressed by many groups (53). The implementation of these recent breakthroughs to thin films, as well as the use of successful passivation schemes developed for PC films, are longed for and highly promising for the future of SC solar cells. SC perovskite remain interesting in multiple ways, for their superior stability, and the possibility they offer to build devices on specific crystallographic planes and on facets presenting homogenous chemical termination. This will be discussed in the next section.

\section{Potential and opportunities for SC beyond PC solar cells}

\section{Anisoptropy in optoelectronic properties and performance}

\subsection{Anisotropy in MAPX}

Single crystals typically have anisotropic properties (physical, thermal and optoelectronic) (148). As an example, in silicon single crystals the hole mobility in the [110] direction is $78 \%$ higher than that in the [100] direction $(148,149)$. As a consequence, the crystal orientation must be taken into account for an optimal device design. In Figure 19 it is displayed the atomic structure of MAPI SC for different crystallographic directions. In addition, a given facet can have different chemical terminations, as shown in Figure 19f, and therefore can influence macroscopic properties, such as SRV, and impact device performance. It is thus highly important to understand how the physico-chemical properties of MAPX depend on orientation and/or termination, and how this anisotropy can be exploited to design better devices.

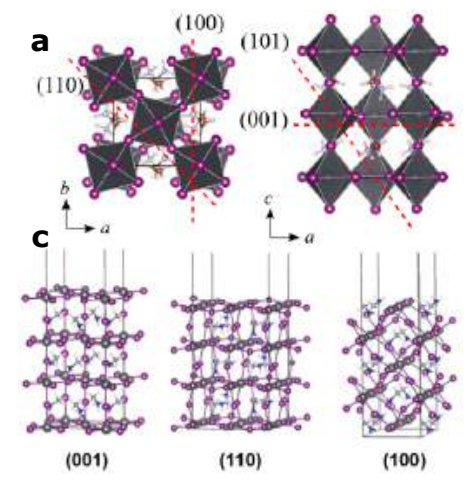

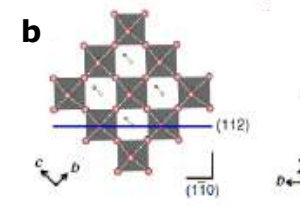

d

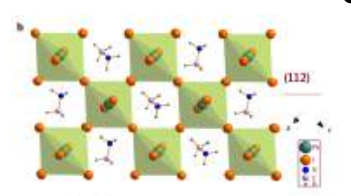

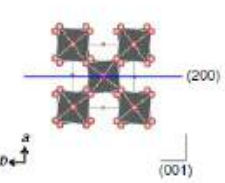

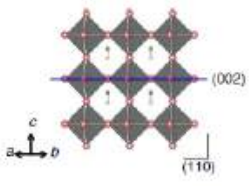

e

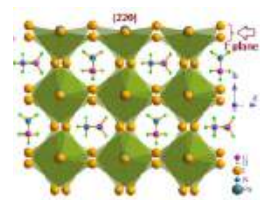

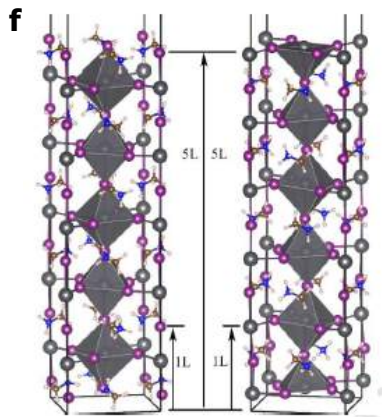

Figure 19 - Tetragonal phase of MAPI showing different orientations and terminations. a) (110), (100), (101) and (001) planes, indicated by the red dashed lines (from ref (150)). b) (112), (200) and (002) planes, indicated by blue lines (from ref (151)). c) Slab along the [001], [110] and [100] direction (from ref (23)). d) Slab along the [112] direction (from ref (152)). e) Slab along the [220] direction (from ref (153)). f) 5 layer slab (5L) showing the MAI termination (left) and $\mathrm{PbI}_{2}$ termination (right) of a (001) facet(from ref (154)). Grey/green octahedron represent $\mathrm{PbI}_{6}$ units.

Early reports on the impact of crystal orientation on device performance were obtained by probing different grains in PC perovskite films. In one example, conductive atomic force microscope (cAFM) was used to measure the I-V characteristic of a $\mathrm{MAPbI}_{3-x} \mathrm{Cl}_{x}$ solar cell with spatial resolution. The results showed the existence of some domains with high efficiency and some with low efficiency, that corresponded to different (seemingly crystal) domains in topographical images (Figure 20a) (155). In MAPI PC thin films an anti-correlation between $\mathrm{J}_{S C}$ and $\mathrm{V}_{O C}$ levels was found for different crystal domains, probably related to an orientation-dependent mobility that promoted high photocurrent at the cost of higher surface recombination (Figure 20b) (156). 
a
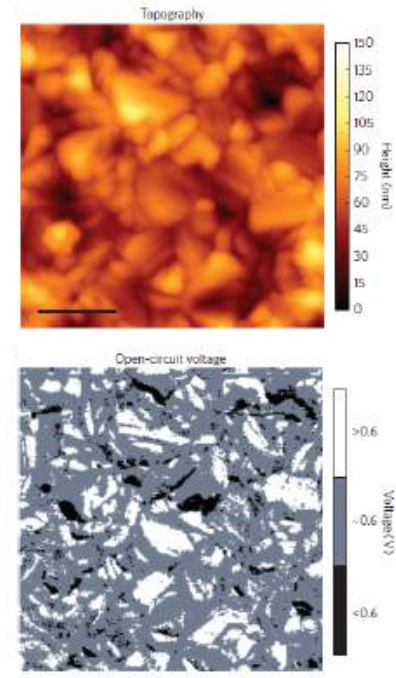
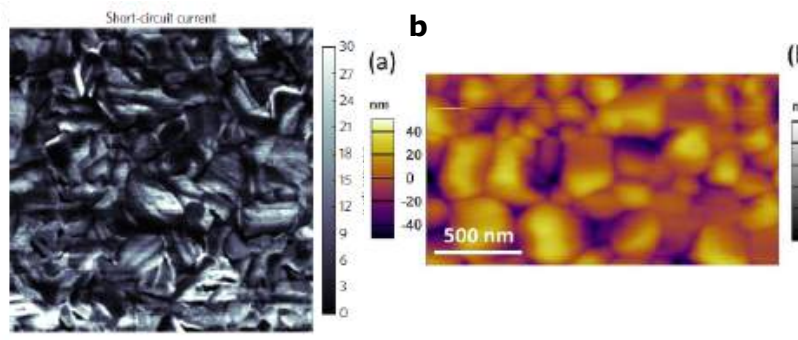

(b)
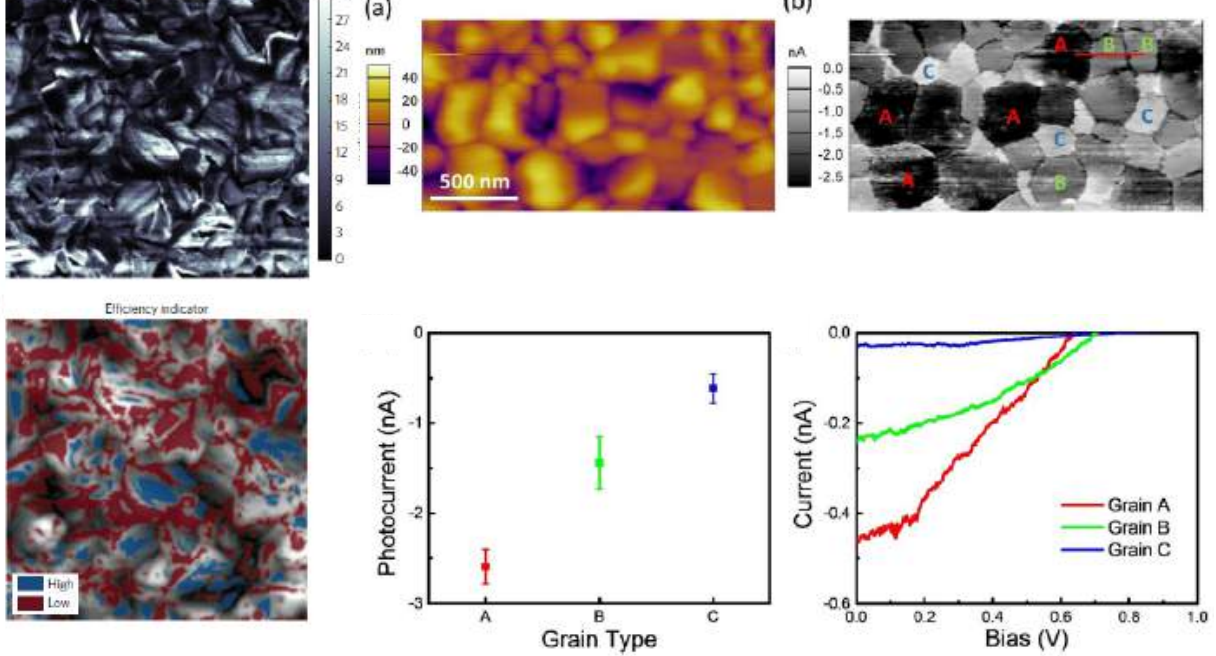

Figure 20 - Anisotropic performance between grains of PC-MAPX. a) Topography image corresponding to the other maps (scale bar is 500nm), short-circuit current $\left(\mathrm{I}_{S C}\right)$ map measured at $0 \mathrm{~V}$ bias under illumination, opencircuit voltage $\left(\mathrm{V}_{O C}\right)$ map measured under illumination relative to a forward reference bias of $0.6 \mathrm{~V}$, and efficiency map with areas in blue (high $\mathrm{V}_{O C}$ and $\mathrm{I}_{S C}$ ) and areas in red (low $\mathrm{V}_{O C}$ and $\mathrm{I}_{S C}$ ) are overlaid on the local topography, indicating that many grains have a specific crystal facet that provides higher performance (from ref (155)) b) Topology and photocurrent map of MAPI/PEDOT:PSS/ITO under the illumination of a green laser while applying a $-2 \mathrm{~V}$ bias to the sample. The three discrete photocurrent levels correspond to grains of different types, as marked by A, B, and C. Average and standard deviation of photocurrent measured on the grains of different types, and averaged point J-V curves at ten different locations for each grain type under illumination (from ref (156)).

Multiple approaches were developed to synthesize PC films with preferred crystal orientation to take advantage of the anistropy. Control over the temperature $(151,157)$ or over the drying process $(158)$, the use of selective solvents (159), the addition of additives to the precursors solution $(160,161)$, space confined methods such as bar coating (162) or post treatments (163), among other techniques were found to promote orientation of the grains towards a unique crystallographic direction. Films were integrated in vertical solar cells, and resulted in an enhanced PCE in all cases. It was concluded that a preferred orientation could improve cell performance $(157,159,160,163)$ compared to a non-oriented PC films, but evidence on which orientation is to be preferred remains inconclusive. This is due to the fact that many of the techniques used to obtain an oriented film (i) have been reported to passivate the surface $(93,139)$ and (ii) often resulted in increased grain size, improved film coverage and crystallinity compared to the reference sample.

As will be discussed in the next section, investigation of anisotropy on SC enables to drastically reduce such experimental bias, as bulk SC commonly exhibit facets of different orientations, allowing one to compare properties and performance of different crystallographic planes and ultimately correlate crystal orientation to optoelectronic properties.

\subsection{Impact of anisotropic charge transport on properties and performances}

Figure 21 summarizes optoelectronic properties probed on different crystal facets for MAPX SC fabricated and characterized in similar conditions, unambiguously demonstrating the existence of anisotropy in the optoelectronic properties of MAPX $(62,63,164,165)$. 
MAPI

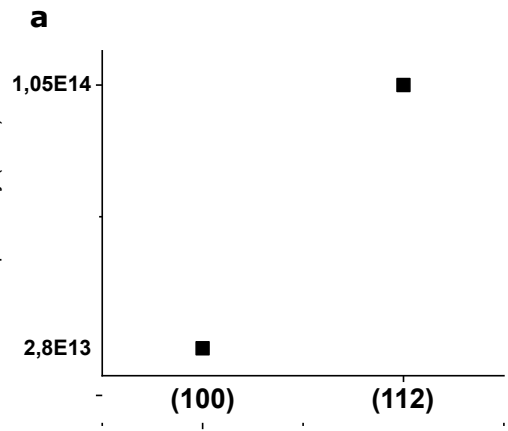

b

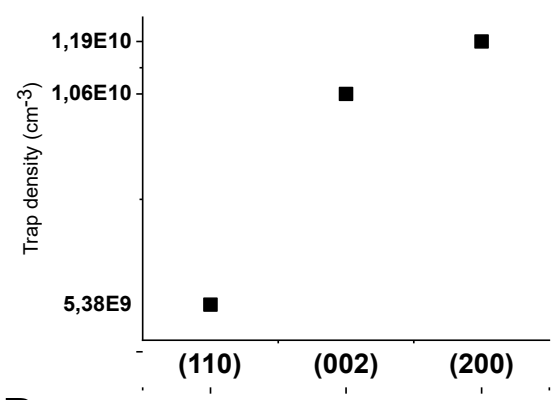

MAPB

C

d
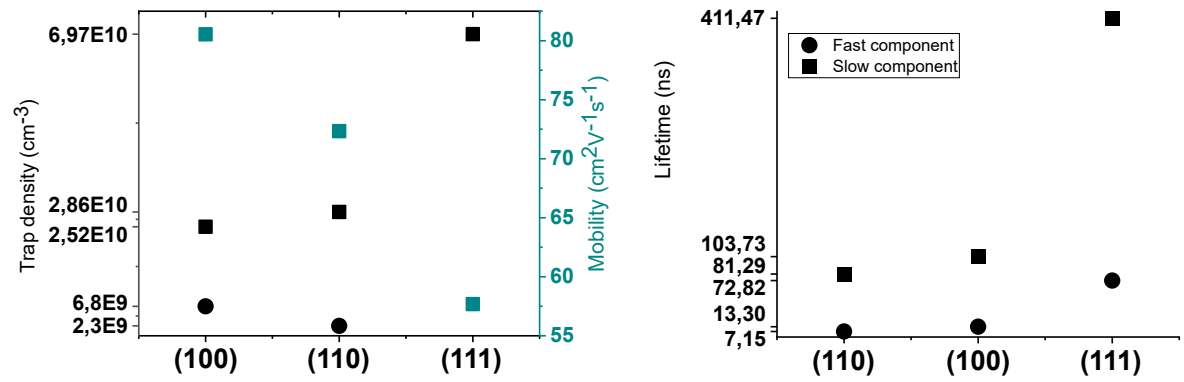

Figure 21 - Anisotropic optoelectronic properties measured on MAPX-SC. a) Trap density of MAPI (100) and (112) facets (data from ref (164)) b) Trap density of MAPI (110), (002), (200) facets (data from ref (165)) c) Trap density and mobility of MAPB (100) (110) (111) facets (data from ref $(62,139)$ ) d) Lifetime of MAPB (100) (110) (111) facets (data from ref (63)).

MAPI In bulk MAPI-SC grown by ITC, the (100) facet displayed a trap density $\left(2.8 \cdot 10^{13} \mathrm{~cm}^{-3}\right) \quad 4 \mathrm{x}$ lower than the (112) facet and higher photoluminescence (Figure 21a) (164), and for MAPI SC grown by AVC, the (110) facet was shown to have a trap density $\left(5.4 \cdot 10^{9} \mathrm{~cm}^{-3}\right) \quad 2 \mathrm{x}$ lower than (002) and (200) facets (Figure 21b) (165). While bulk SC can hardly be integrated at such in solar cells, it is possible to build planar photodetectors (PD) by depositing gold electrodes on one of the naturally exposed facet to evaluate the impact on device performance. The (110) plane exhibited the lowest dark current, the lowest $\mathrm{R}$ and an on/off ratio as large as 342 under $0.5 \mathrm{~V}$ bias, that is five-fold larger than that of the (002) device and ten-fold that of the (200) device as shown in Figure 22a and b (165). In addition, larger photocurrents, responsivity and EQE values were reported for PDs built on the (112) and (220) compared to the (100) facet (Figure 22c, d, e and f) (152, 153). By using a top-down strategy to expose the (001), (100) and (112) facets, vertical PDs showed lower mobility along the [001] direction compared to the [100] and [112] direction both for holes and electrons (166). 

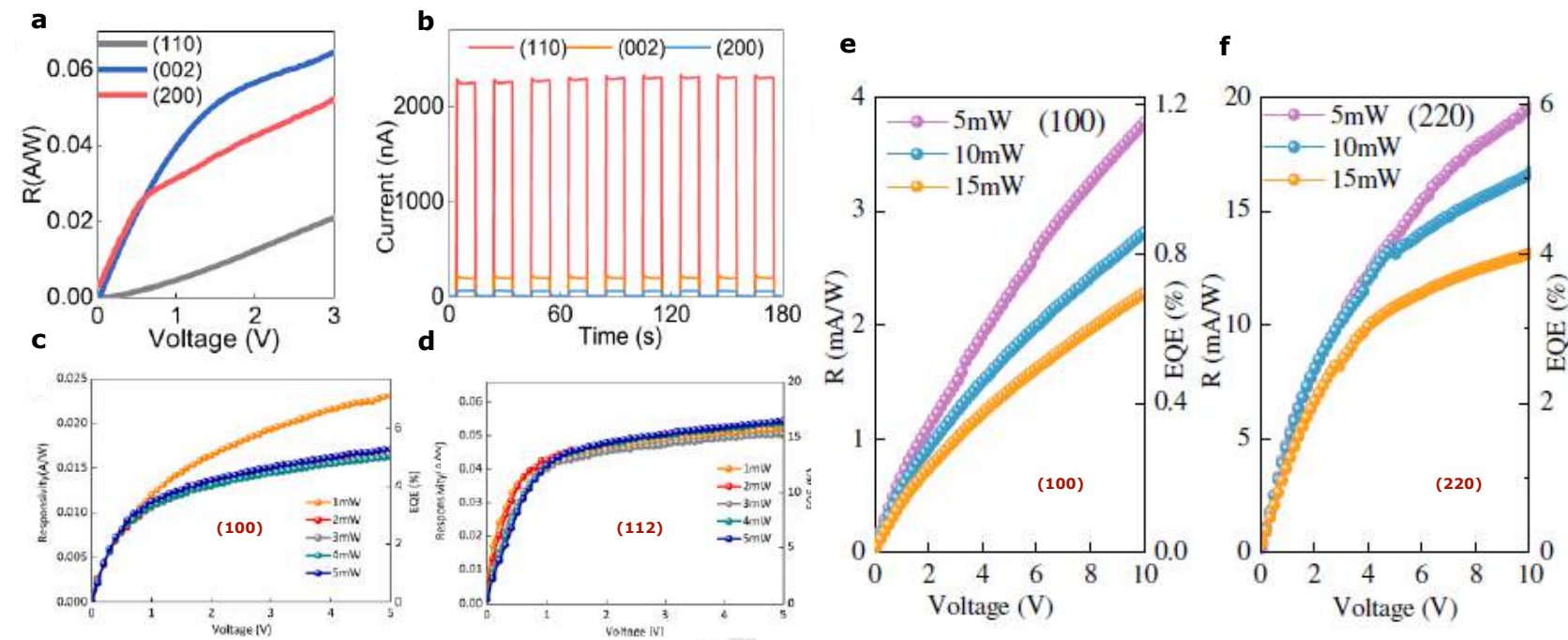

Figure 22 - Performance of planar and vertical PD built on different facets of MAPI-SC. a) Responsivities and detectivities b) Continuous on-off circles of the (002), (110) and (200) PD under $1 \mathrm{~mW}$ illumination (from ref (165)). Responsivities and EQEs of (c) (100) and (d) (112) PD (from ref (152)). Responsivities and EQEs of (e) (100) and (f) (220) facet PD (from ref (153)).

MAPB Metal-semiconductor-metal (MSM) PDs showed better performance (lower dark current, higher photocurrent and $>2 \mathrm{x}$ higher responsivity) if the device was built on the (110) facet compared to the (100) facet (Figure 23a and b) (167). Similar conclusions were obtained by comparing trap density (Figure 21c, black circles) and responsivity of X-ray detectors $(\mathrm{Au} / \mathrm{MAPB} / \mathrm{C} 60 / \mathrm{BCP} / \mathrm{Cr})$ of two MAPB SC grown in slightly different precursor solution in order to tune crystal orientation (Figure 23d) (139).

If the facets were instead obtained by a top-down method, different results were observed, with (100) facets reported to be superior over the (110) and (111), both in terms of trap density (Figure 21c, black squares) and mobility (turquoise squares)(62), or, in another study, the (111) facet superior in terms of lifetime over the (100) and (110) facets (Figure 21c) (63). This suggests that the growth conditions may have major impact on the properties.

MAPC Planar photodetectors were also built on MAPC single crystal naturally exhibiting (100) and (110) planes. Cheng et al. reported similar $\mathrm{R}$ and $\mathrm{EQE}$ for both facets but observed that the dependence to voltage was substantially different, confirming the existence of different transport mechanisms in (100) and (110) planes for MAPC crystals as well (Figure 23e and f). The trap density was reported to be lower for the (100) facet compared to the (110) facet (148). 

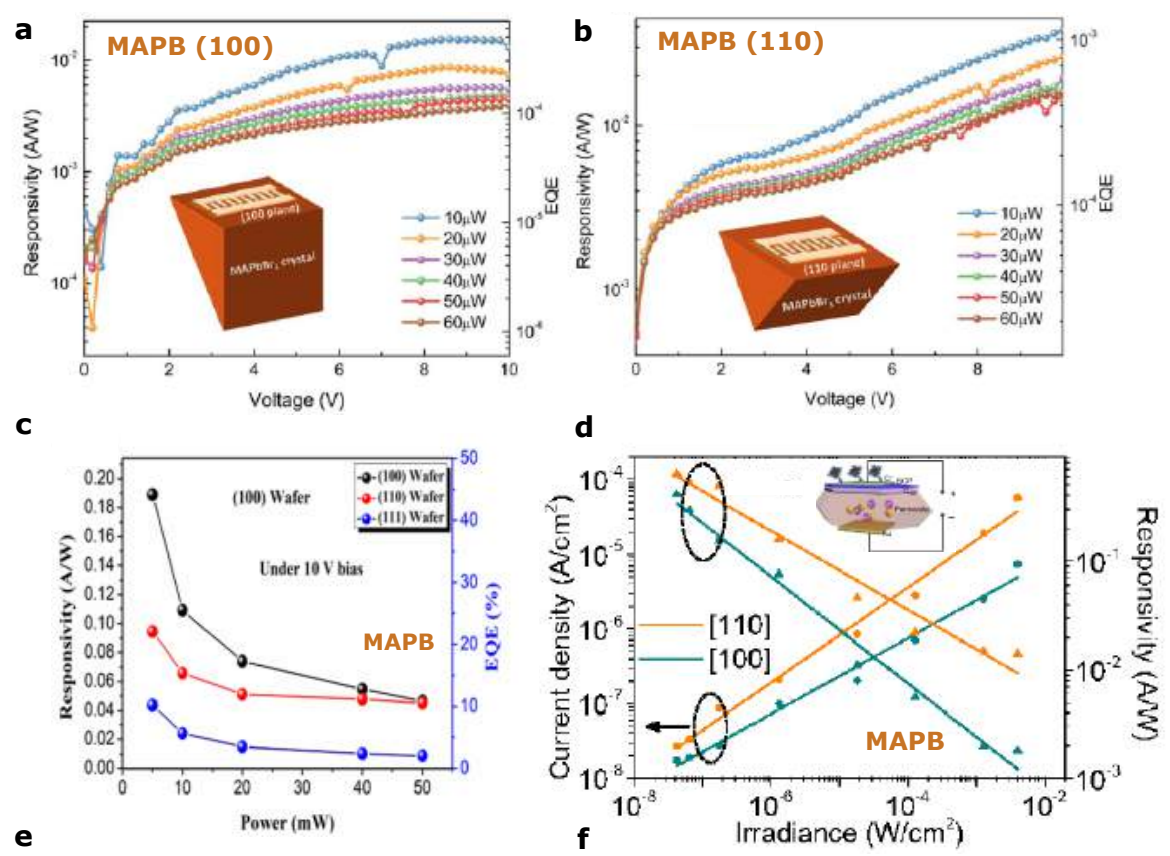

e
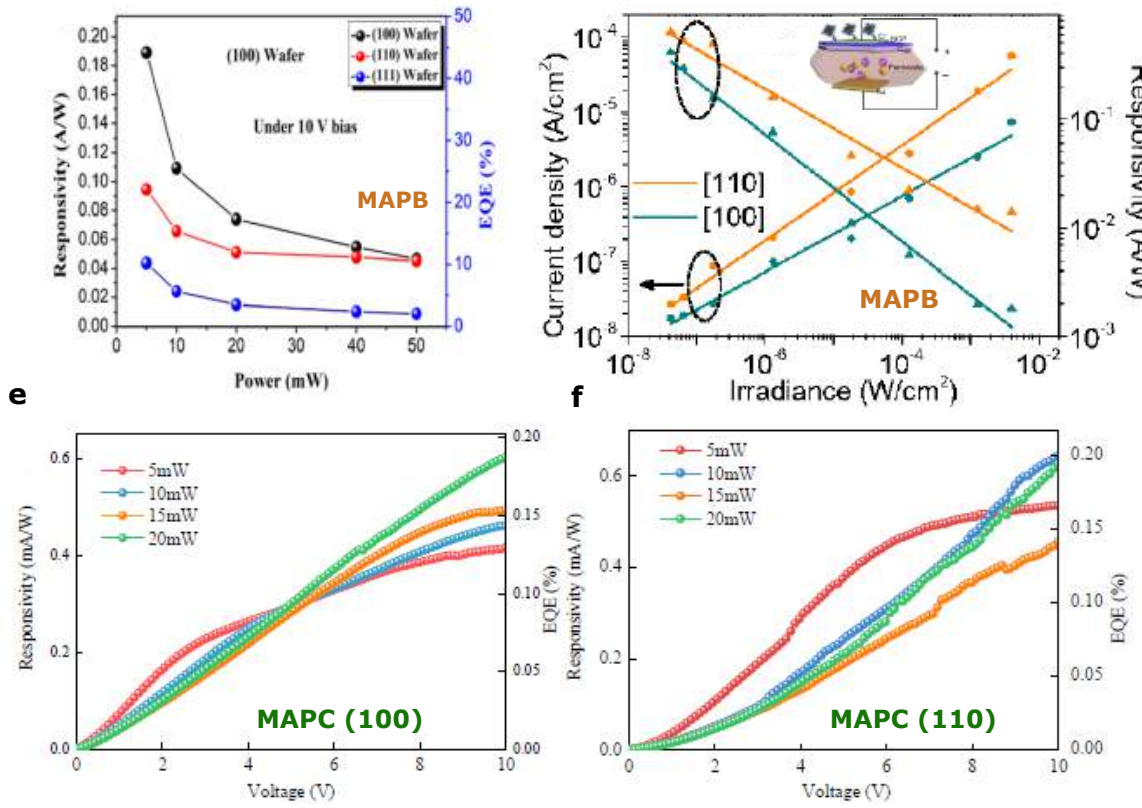

Figure 23 - Performance of planar and vertical PD built on different facets of MAPB and MAPC-SC. Responsivities and EQEs of (a) (100)-PD and (b) (110)-PD. Simple diagrams of device design and fabrication location are inserted (from ref (167)). c) Responsivity (R) and external quantum efficiency (EQE) of (100)-PD, (110)-PD, (111)PD under differently oriented illumination powers at $10 \mathrm{~V}$ bias (from ref (63)). d) Current density and responsivity of (110) and (100) vertical PD versus irradiance and the device structure of MAPB SC X-ray detector (from ref (139)). Responsivities and EQEs of MAPC (e) (100)-PD and (f) (110)-PD (from ref (148)).

In all cases, the TFL regime appeared at very different voltages for different orientations, suggesting different trap states for different facets (Figure 24) $(63,148,164)$.
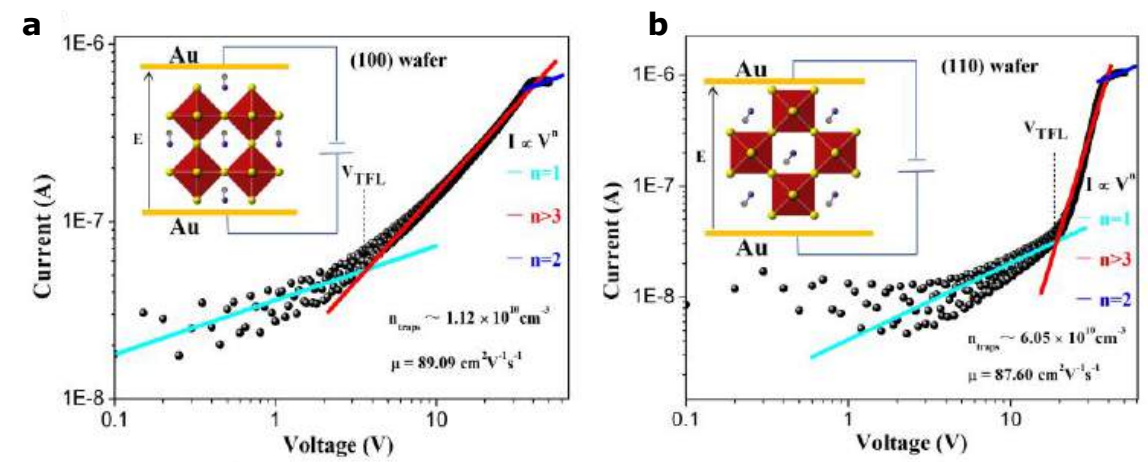

Figure 24 - TFL regime appearing at different voltages for different facets. Typical dark current-voltage curves of the space charge-limited current (SCLC) devices on a) (100) and b) (110) MAPB crystal wafers (from ref (62)).

In addition, the optical constants $\mathrm{n}$ and $\mathrm{k}$ for a MAPB crystal were reported to be anisotropic (63).

The main conclusion on anisotropic charge transport, properties and performances are summarized in table 6 , along with hypothesis/explanations on the origin of the anisotropy from the authors. 


\begin{tabular}{|c|c|c|c|c|}
\hline Material & Properties studied & Conclusion & Hypothesis & Ref \\
\hline MAPB & $\begin{array}{c}\text { Photocurrent, } \\
\text { R, EQE }\end{array}$ & $(110)>(100)$ & - & (167) \\
\hline MAPB & $\begin{array}{c}\text { Photocurrent, } \\
\text { R, EQE, Lifetime }\end{array}$ & $(100)>(110)>(111)$ & $\begin{array}{l}\text { Anisotropic ion migration } \\
\text { built in electric field }\end{array}$ & (63) \\
\hline MAPB & $\begin{array}{l}\text { Trap density, PL, } \\
\text { mobility, photocurrent, } \mathrm{R}\end{array}$ & {$[110]>[100]$} & 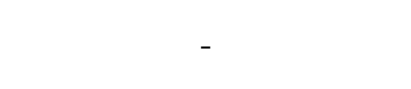 & $(139)$ \\
\hline MAPB & $\begin{array}{l}\text { Mobility, } \\
\text { trap density }\end{array}$ & $(100)>(110)>(111)$ & $\begin{array}{l}\text { Anisotropic defect } \\
\text { migration }\end{array}$ & $(62)$ \\
\hline MAPI & $\begin{array}{l}\text { Photocurrent, } \\
\text { R, EQE }\end{array}$ & $(220)>(100)$ & $\begin{array}{l}\text { Anisotropic ion } \\
\text { migration }\end{array}$ & (153) \\
\hline MAPI & $\begin{array}{l}\text { Photocurrent, } \\
\text { R, EQE }\end{array}$ & $(112)>(100)$ & $\begin{array}{l}\text { Enhanced rotational } \\
\text { freedom for } \mathrm{MA}^{+} \\
\text {in (112) plane }\end{array}$ & $(152)$ \\
\hline MAPI & $\begin{array}{c}\text { Trap density, } \\
\text { Photocurrent, R, EQE }\end{array}$ & $(110)>(002)>(200)$ & $\begin{array}{l}\text { Anisotropic ion migration } \\
\text { built in electric field }\end{array}$ & $(165)$ \\
\hline MAPI & Mobility & $\begin{array}{l}{[100]>[001]} \\
{[112]>[001]}\end{array}$ & $\begin{array}{l}\text { Anisotropic ion } \\
\text { migration }\end{array}$ & (166) \\
\hline MAPI & $\begin{array}{l}\text { PL, trap density, } \\
\text { ionic movement }\end{array}$ & $(100)>(112)$ & $\begin{array}{l}\text { Anisotropic ion } \\
\text { migration }\end{array}$ & $(164)$ \\
\hline MAPC & $\begin{array}{l}\text { Trap density } \\
\text { R, EQE }\end{array}$ & $\begin{array}{l}(100)>(110) \\
(100) \propto(110)\end{array}$ & $\begin{array}{l}\text { Anisotropic charge } \\
\text { densities and } \\
\text { distributions }\end{array}$ & $(148)$ \\
\hline
\end{tabular}

Table 6 - Conclusion of the orientation dependent properties of single crystals experiments. Charges traveled parallel to the probed plane when the crystallographic plane is indicated with brackets (planar devices) and perpendicular to the surface when indicated with square brackets.

Ion migration is an highly anisotropic process $(103,168)$ as it depends on the ionic density of a given crystallographic direction. For example in MAPI the average density of $\mathrm{I}^{-}$ions on the (220) plane is larger than that of the (100) plane, whereas (112) plane offered easier ion hopping compared to the (100) due to smaller sites distance. This has implications on the interpretation of results due to the anisotropy of the ionic current that is typically present as a transitory in device characterization (Figure 25a and b). Ion and electron conductance along the (112) plane were found to be respectively 19 times and 11 times higher than along the (100) plane (169). Mobile ions are also responsible for the formation of an anisotropic built-in field that can affect electronic and ionic transport through the crystal $(63,165)$. Crystallographic planes such as (110) and (002) (MAPI) presenting a mix of positive and negative ions are thought to hinder ionic migration and limit the formation of a built-in field, and could enable better/faster photo-response of devices, unlike the (200) plane which is $\mathrm{I}^{-}$rich (165). 


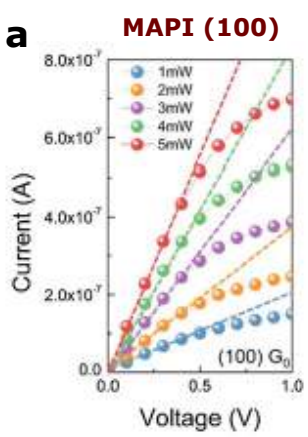

d

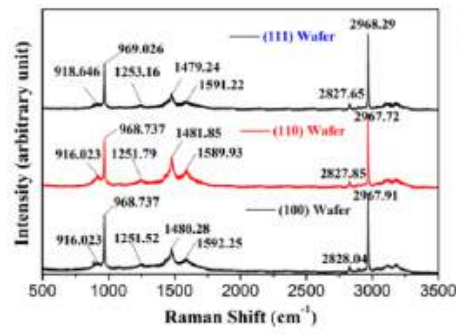

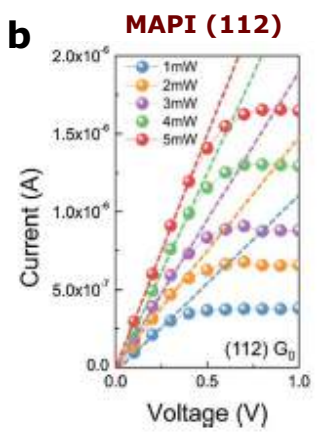

e

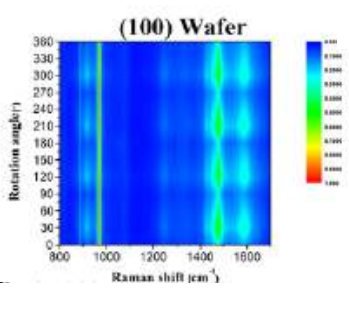

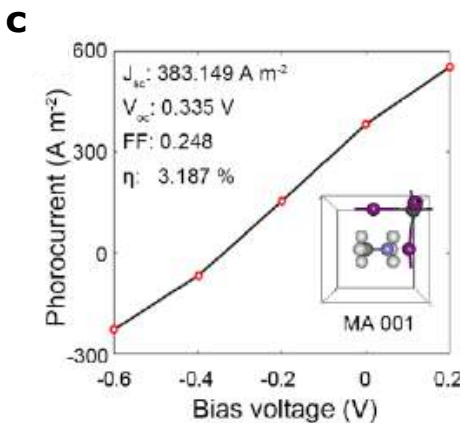

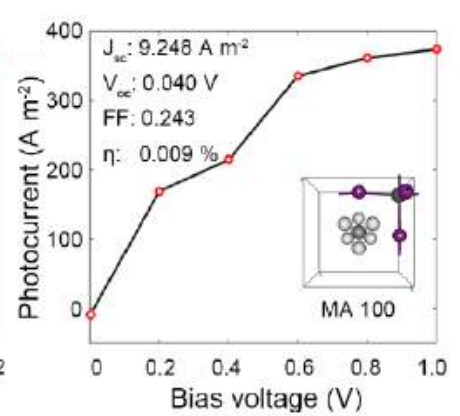

Figure 25 - Insights on the origin of MAPX anisotropic properties. Original mixed conductance $\mathrm{G}_{0}$ on MAPI (a) (100) and (b) (112) planes illuminated by a continuous $405 \mathrm{~nm}$ laser with various light power densities (from ref (169)) c) Current generated at different bias voltages for different MA orientation (from ref (170)) d) $\mathrm{MA}^{+}$raman spectra signal from MAPB-SC (100), (110) and (111) wafers, measured without polarizer in optical path with an excitation wavelength of $633 \mathrm{~nm}$. e) Angle-resolved polarized Raman spectra of differently oriented MAPB wafers (from ref (63)).

Higher mobility and higher holes/electrons velocities were predicted in MAPI if $\mathrm{MA}^{+}$ions were oriented parallel to the transport direction, with a potential impact on photocurrent (Figure 25c) (170), suggesting an anisotropic hole effective mass (171), as for silicon single crystals (139). Experimental evidence of the anisotropy induced by $\mathrm{MA}^{+}$orientation in MAPB was recently given by probing different crystallographic orientation of a MAPB SC with Angle-Resolved Polarized Raman spectroscopy. Except for the (111) plane, the intensity of Raman peaks for (100) and (110) planes displayed periodic variation as the polarization was changed from 0 to $360^{\circ}$ (Figure 25d and e), suggesting in-plane anisotropy of MAPI crystals caused by $\mathrm{MA}^{+}$orientation (63).

Overall, the understanding of the effect of ionic migration and $\mathrm{MA}^{+}$orientation on electronic charge transport is still in its infancy, and needs to be explored further.

It is difficult to draw definitive conclusions on which crystal orientations are to be preferred for device design, due to differences in growth method employed across literature that can result in different chemical termination, that were not taken into account in the studies, the contribution of the ionic current to the total current and other experimental bias. Further research to assess which crystallographic direction are the most interesting for charge transport are highly needed.

\subsection{Facets/termination dependent energy levels and charge transfer}

Optimal alignment of energy levels between the perovskite and the charge transport layers or electrodes is key to high efficiency solar cells, with a reported optimum band offset of $0.2 \mathrm{eV}$ (61). The energy levels of the conduction and valence band for perovskites have been reported to depend on the atomic termination of the surface $(172,173)$, as well as on the type of crystallographic facet. In particular, for MAPI SC a work function of $4.6 \mathrm{eV}$ for the (112) plane and of $4.7 \mathrm{eV}$ for the (100) plane has been reported (164). In addition, the termination of the (001) facet can either be MAI or $\mathrm{PbI}_{2}$ terminated (Figure 26b). It was found that the energy levels of the $\mathrm{VB}$ and the $\mathrm{CB}$ of the $\mathrm{PbI}$ terminated surface were $\sim 1 \mathrm{eV}$ below those of the MAI terminated surface, revealing the critical role played by the surface termination (Figure 26c) (174). As a consequence, different band offsets are produced for different facets in contact with a given transporting material, which impacts charge transfer $(164,174)$. Combining ab initio calculations and photoelectron spectroscopy it was found that charge transfer in MAPI depended on the facet in contact with the hole transport layer. In particular, non-polar surfaces (001) and (110) tended to to favor hole injection into Spiro-OMeTAD while the more polar (100) surface facilitated electron transfer to PCBM (23). Evidence of the impact of crystal orientation on charge transfer from perovskite to a CTL was found also in oriented PC MAPI films, with (002)/(110) films showing better charge transfer to spiro-OMeTAD and to $\mathrm{TiO}_{2}$ while (112)/(200) films showing better charge transfer to PEDOT:PSS and PCBM (Figure 26a and b) (175). For (001) facets, the charge extraction at a perovskite/ $\mathrm{C}_{60}$ interface was predicted to be spontaneous (CB energy higher than LUMO) only if the material was MAI-terminated (174). In addition, shallow surface states for (110) and (001) PbI-terminated surfaces just above the VB could act as intermediates 
for hole transfer between the perovskite and a HTL and thus be beneficial for solar cell performances (150). Inversely, MAI-rich layers were reported to have a more favorable energy level alignment for electron extraction to a fullerene ETL (25).

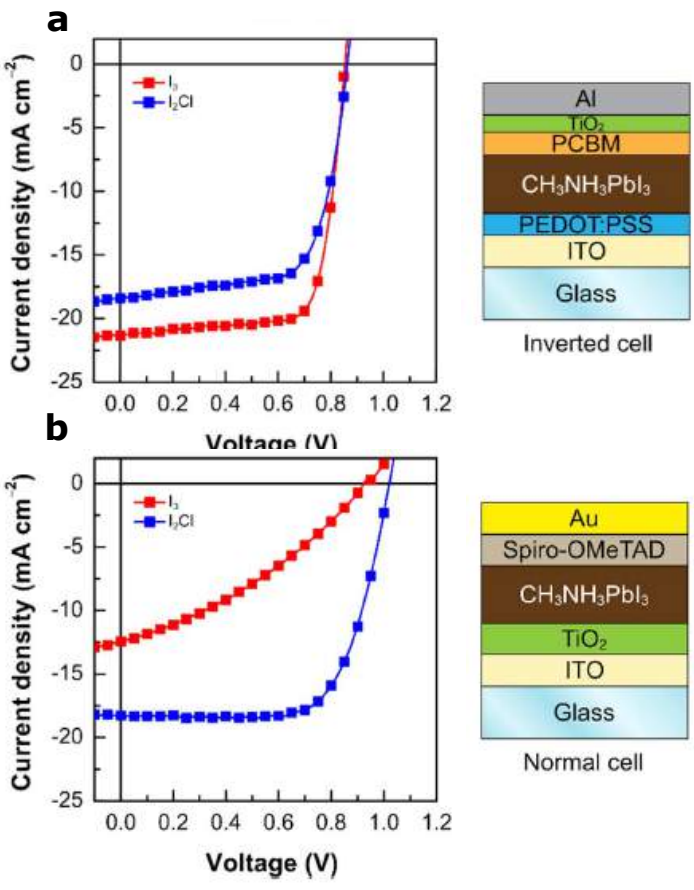

C

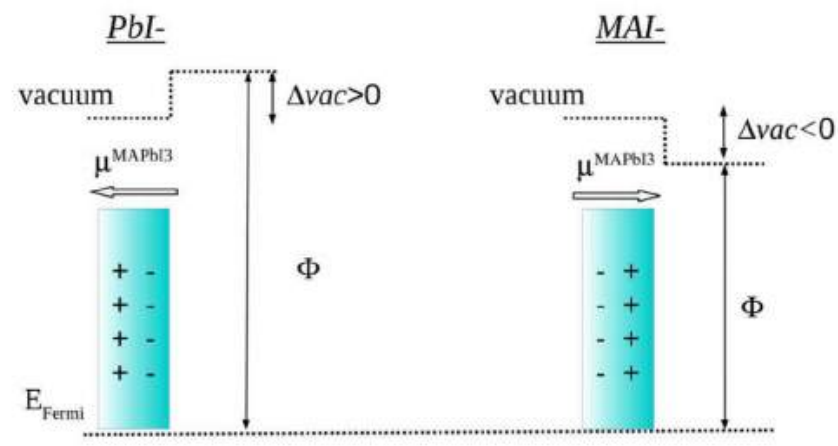

Figure 26 - Anisotropic charge transfer. Device structures and J-V curves of a) inverted cells and b) normal cells measured under AM 1.5G condition (from ref (175) c)Shift of the vacuum level on MAPI slab model due to the surface dipole associated to the surface termination (from ref (174).

In summary, the extraction of charges from the perovskite layer to the transport materials depends both on the orientation and the atomic termination. As a consequence, to achieve ultimate performance it is important to (i) investigate how crystal orientation impacts solar cell performance without neglecting surface composition, (ii) develop synthesis to choose $\grave{a}$ la carte the orientation of monocrystalline thin films and (iii) develop interface engineering strategies that takes into account of the surface energetics of single crystals facets. We will develop some of these concept in the next section.

\section{Perspectives and pathways for MAPX SC solar cells}

\subsection{Surface recombination}

MAPX SCs have outstanding bulk properties, but if not passivated, surface recombination becomes the dominant channel for non-radiative recombination, as evidenced in section 6 . This is especially true after integration in devices, because all electrons and holes generated in the bulk have to travel through the surface to be extracted. In order to reduce the efficiency losses associated with charge recombination at the surface, much efforts should be put in achieving surfaces with the lowest defect density. One approach consists in optimizing synthesis protocols, taking advantage of low temperature growth and exploring different solvents to reduce trap densities originating from dissolving ions (29), which could also have a beneficial impact on stability by limiting degradation pathways. In addition, as discussed in section 7, the anisotropy of SCs can be a strategic asset in designing devices with better performance. As a consequence, growth and characterization methods are essential to gather information needed for an improved design. Focus on the development of growth methods to control the orientation and the chemical termination is thus highly desired to customize SCs surfaces prior to their integration in solar cells. 

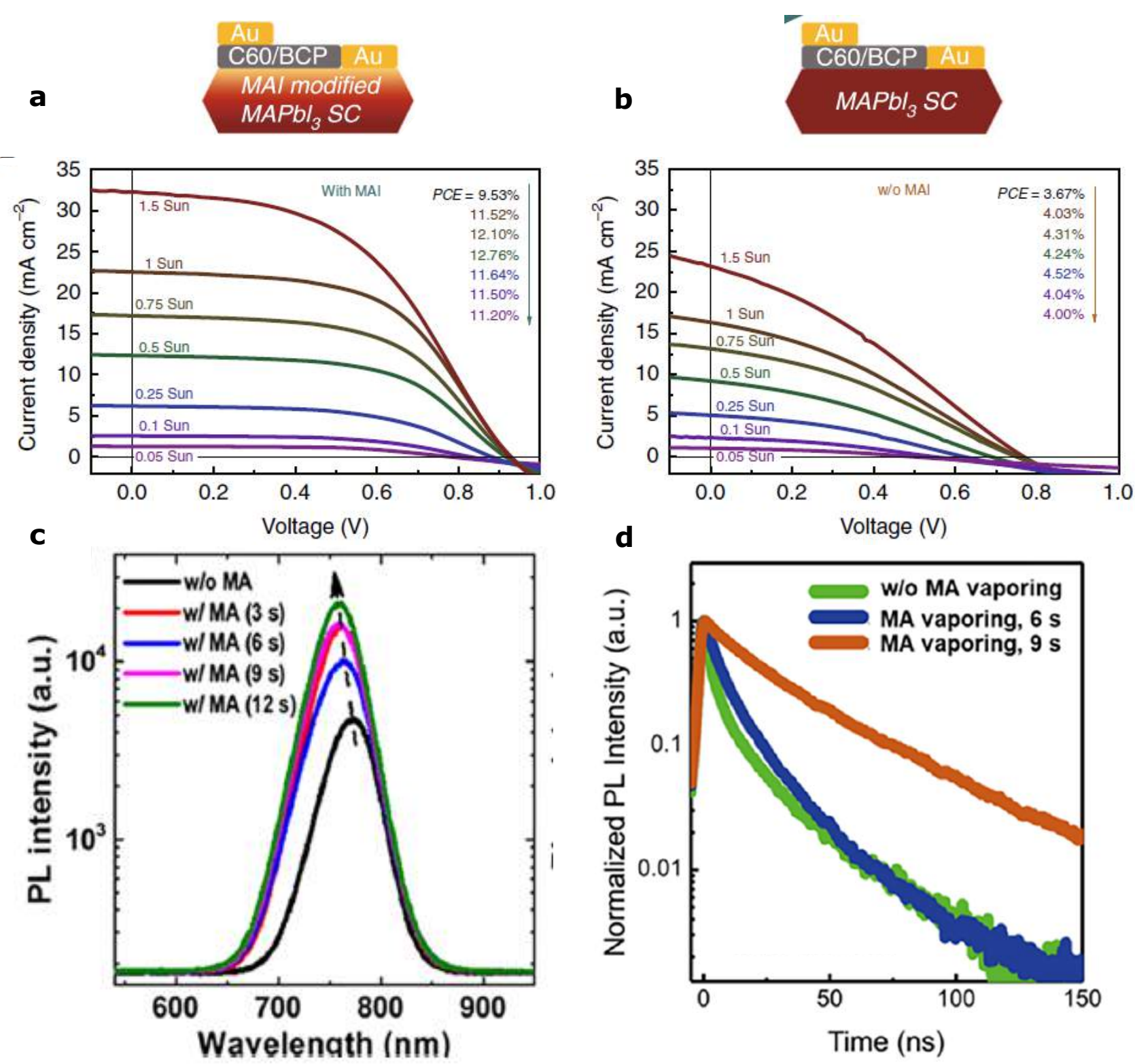

Figure 27 - Surface passivation of MAPX-SC by chemical treatments. By simply treating a MAPI single crystal with MAI, Song et al. obtained a champion cell retaining $99.77 \%$ of his initial efficiency after 200 hours of continuous operation under 1 Sun and almost twice as efficient as other SC lateral cells (139) as illustrated by the J-V curves under different light intensity of devices with (a) and without (b) MAI treatment (from ref.(139)). Zhou et al. passivated MAPI SC surface traps using methylamine (MA) vapors. Such passivation of surface traps resulted in an elongated lifetime as illustrated with the steady-state PL measurements in (c) and increased PL intensity as shown in the normalized PL decay kinetics of freshly grown SC exposed to MA vapor for different times (d)(from ref (176)).

Surface passivation must also be studied in greater detail to further improve surface properties, and it was shown to be successful in PC solar cells by making surface states electronically inactive. In SC solar cells, chemical techniques based on a lewis acid-base approach (139) or using pyridine (132) have proven effective for passivation, reducing surface traps and improving lifetime and PL intensity. In particular, simple MA or MAI treatments were found to greatly improved performances (Figure 27) (139, 176).

Other passivation techniques involve the creation of a protecting layer on top of the crystals. Yang et al. for example created a thin lead (II) oxysalt on top of MAPI SC by reacting the surface with sulfate or phosphate ions as illustrated in Figure 28a. Such layer could protect the material against moisture as was evidenced by dipping the sample in water: the untreated crystal turned yellow within 10 seconds because of the decomposition of the surface into $\mathrm{PbI}_{2}$, whereas the passivated sample remained black after more than 60 seconds (Figure 28b) (177). Duim et al. showed that, when reacted with benzylammonium cations, MAPB SC surface turned into the more stable $(\mathrm{BA})_{2} \mathrm{PbBr}_{4} 2 \mathrm{D}$ perovskite through the replacement of $\mathrm{MA}^{+}$ions by $\mathrm{BA}^{+}$ions (Figure $28 \mathrm{~d}$ ). Such passivating layer resulted in 5-fold enhanced photoluminescence intensity and more than 3-fold increase in carrier lifetime (Figure 28e and f) (178). Similarly, Lu et al. fabricated a MAPB-MAPC heterojunction on the 
surface of MAPB single crystal by simply heating MAPB SC with MACl powder in a glovebox. The passivating effect of such layer was evidenced by a one fold increase in lifetime (Figure 28c) (146).

a

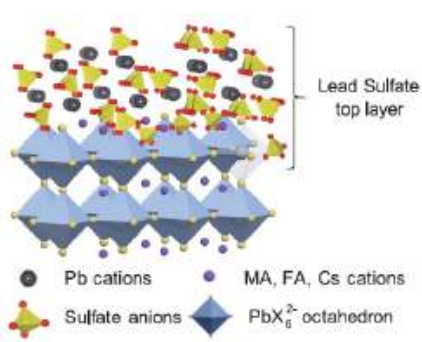

d
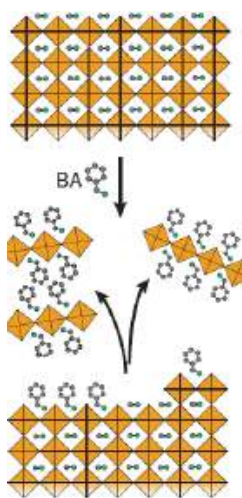

b

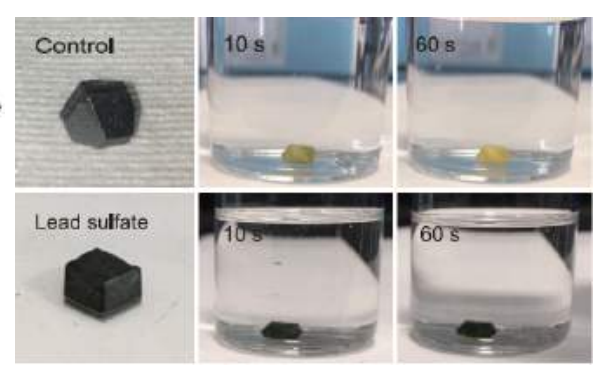

e

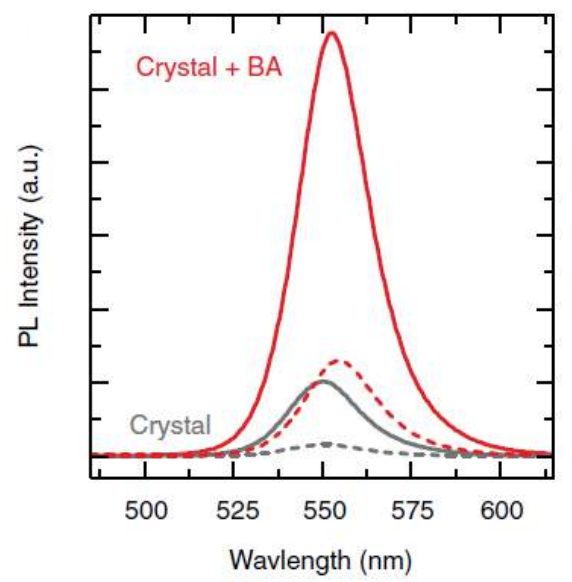

C

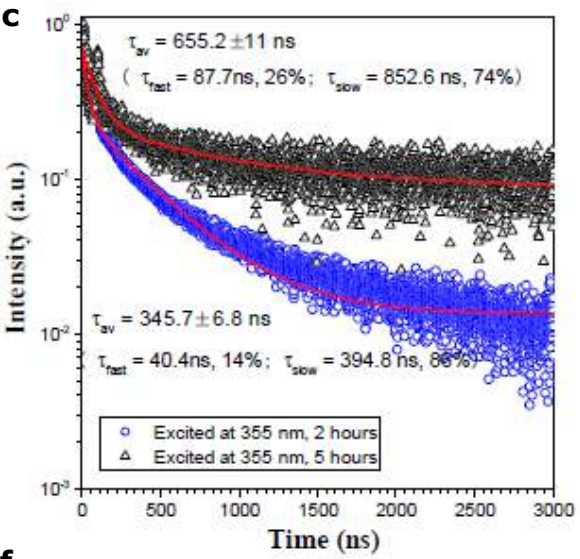

f

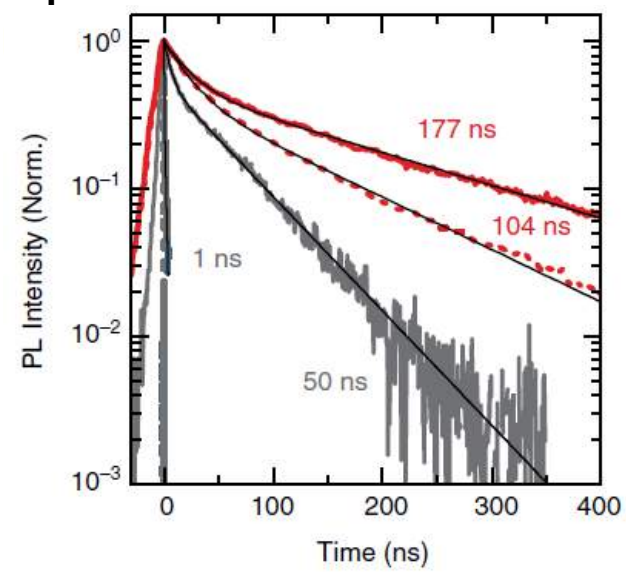

Figure 28 - Optimisation of MAPX-SC surface by a protecting layer. a) Schematic illustration of in situ formation of a lead sulfate top layer on the perovskite surface. b) MAPI-SC without and with lead sulfate top layers dipped into water at different time intervals (from ref(177) c) Lifetime of MAPB-SC with $\mathrm{MAPC}_{3}-\mathrm{MAPB}_{3}$ heterojunction excited at $355 \mathrm{~nm}$ (from ref (146)). d) Schematic of the formation of $(\mathrm{BA})_{2} \mathrm{PbBr}_{4}$ at the MAPB SC surface. e) Steadystate photoluminescence spectra (averaged over 5 spots) obtained from the crystal surface of a sample that has been treated with benzylamine (BA) ligands in chlorobenzene (red line) and an untreated crystal (gray line), both in air (solid lines) and in vacuum (dashed lines) f) Time-resolved decay profiles of the same samples fitted by a biexponential function (black lines), labels indicate the extracted average lifetimes (from ref (178).

Other methods developed for PC perovskite such as PMMA passivation, 2D perovskite capping, fullerene and $\mathrm{Al}_{2} \mathrm{O}_{3}$ post treatments, addition of passivation agent during the synthesis, among many other methods (179), might work for SC as well and thus accelerate SC passivation research (35, 173, 180). In addition, because PC films present different facets and terminations, passivation need to be composed of both lewis bases and lewis acids to passivate both positive and negative defects (181). In SC passivation can likely be tailored for a given crystal orientation and termination and thus be more effective.

\subsection{Energy level alignment}

As discussed in section 7, energy levels offsets at interfaces should be optimized to $\sim 0.2 \mathrm{eV}$ to ensure good charge collection, that set a restriction for the selection of CTL. Energy levels of perovskite SC have been demonstrated to be orientation and termination dependent, which can be an asset in device design (17, 182). Chemical post treatments such as passivation can be used to tune surface energy levels in order to optimize the offset. Polar molecules in particular are able to form dipole interlayers at the perovskite-ETL/HTL interface, pulling down/up the surface vacuum level (183-186). Another approach is to integrate additional layers between the perovskite and CTL. Such interfacial layers can help optimize charge collection by adding new energy levels at the interfaces which reduce interfacial losses commonly observed at large energy offsets interfaces $(27,93,138$, 139, 187, 188), and enabled for higher SC solar cell performance (Figure 29B) $(6,92,105,115)$. Some examples include PS, $\mathrm{Ga}_{2} \mathrm{O}_{3}, \mathrm{LiF}, \mathrm{PMMA}$ or Phenethylammonium Iodide (PEAI), BCP buffer layers, perovskite quantum dots, PFN-P2, PCBM, $\mathrm{C}_{60}$ among many other $(187,189,190)$. Importantly, most interfacial layers allow to improve both charge extraction and stability of the cells $(189,191)$. 
Other strategies such as bulk doping and surface doping were also reported for perovskite PC thin films in order to modify energy levels positions, and could be applied to SC as well. In summary, it is important to understand the impact of environment, orientation, and surface chemistry have on the surface energy levels of perovskite SC to select the ETL/HTL with optimal energy offsets and/or to modify energy levels in accordance.

\subsection{Stability}

Studies on PC films showed that once integrated in devices, many charge transport layers, additives and electrode materials could act as additional sources of degradation (181, 192-196). It is highly likely that SC would suffer the same problem when assembled in a cell with those materials. One reason is the accumulation of MAPX ions that migrate to the interfaces and can react with CTM and electrode materials. Thus it is important to design diffusion barrier to prevent such reaction from occuring $(31,128)$. Such barriers are called ion blocking layers and take the form of post treatment on the perovskite layer before the completion of the cell. Importantly, blocking layers such as PCBM (Figure 29) were also efficient to stop issues related to light illumination or heat for MAPI PC films $(92,197,198)$.

In addition, control over the chemical termination, that can be achieved by optimizing the precursors ratios, could help optimizing energy level alignment in solar cell, but also to achieve higher stability. As an example, in MAPI SCs, MAI-rich surfaces are reported to be thermodynamically more stable than $\mathrm{PbI}_{2}$-terminated surfaces $(154,174)$. Crystal orientation was predicted to impact the stability of ${\mathrm{MAPI}-T i O_{2}}_{2}$ interfaces (199), indicating another pathway for improvement and suggesting that an holistic approach in device design could be a winning strategy.

a

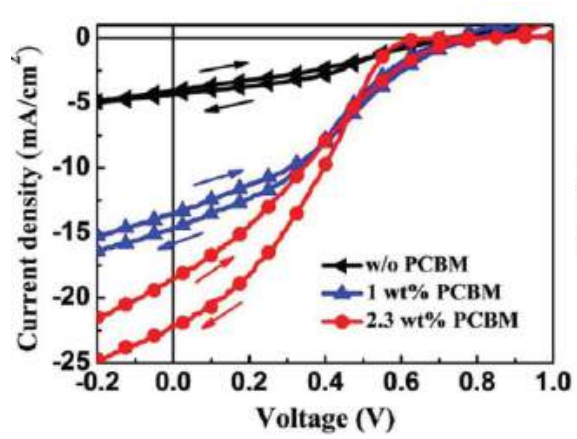

b

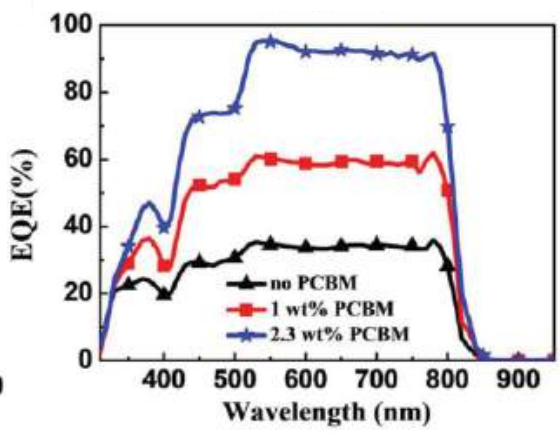

Figure 29 - Interfacial layers. Photovoltaic performances of devices with and without PCBM layer, prepared at various concentrations, showing the importance of the buffer layer. a) J-V curves measured under the illumination of 1 sun (AM1.5G) and b) Corresponding EQE spectra (from ref(92)).

\subsection{High Efficiency Architectures}

The existence of an indirect bandgap $\sim 60 \mathrm{meV}$ lower in energy than the direct bandgap, due to Rashba splitting of the conduction band (24, 200-204), was predicted (205-209) and measured experimentally (Figure 30a) (24, 200-204). Because the absorption coefficient associated to the indirect bandgap is several orders of magnitude lower than that of the direct bandgap, its presence becomes more evident in the absorption spectrum of thicker crystals. In our opinion, this has led to the inaccurate conclusion that MAPC, MAPB and MAPI SCs have a narrower bandgap (2.97 eV (66), $2.16 \mathrm{eV}(117)$ and $1.48 \mathrm{eV}(49)$, respectively) than MAPX PC (3.11 eV, $2.2 \mathrm{eV}$ and $1.55 \mathrm{eV}(210))(36,96,211)$, as the former are typically characterized as thick crystals, whereas the latter as thin films. Corroborating evidence for this can be found in Chen et al., that reported an increase of the absorption edge of a MAPI SC from 790 to $810 \mathrm{~nm}$ when the film thickness was increased from $500 \mathrm{~nm}$ to 10 $\mu \mathrm{m}$ (93). By combining ellipsometric and transmission absorption spectra Wenger et al. calculated the direct bandgap to be $2.31 \mathrm{eV}$ for a MAPB SC (Figure 30b), closely matching that reported for polycrystalline thin films (52).

MAPI-SC solar cells could take substantial advantage of this indirect band gap to absorb lower energy photons and achieve higher theoretical efficiency (the $1.6 \mathrm{eV}$ direct bandgap of MAPI-PC is larger than the optimal $1.3 \mathrm{eV}$ of the Shockley-Queisser (SQ) model). However, due to its indirect nature it has a small absorption coefficient, requiring large material thickness for all photons to be absorbed. The superior optoelectronic properties of SC (6-fold longer diffusion length than PC (52)) allows to use muck thicker films still with optimal carrier collection. The trade-off between increased bulk recombination and increased absorption was predicted for a MAPI thickness as high as $200 \mu \mathrm{m}$, although $10 \mu \mathrm{m}$ MAPI solar cell would already allow for a relative $10 \%$ 
increase of PCE compared to a $500 \mathrm{~nm}$ thick film (Figure 30d) $(30,36,93)$. The same would not be possible for PC solar cells due to the much smaller diffusion length.

This peculiarity could be further exploited by designing smart device architectures to orthogonalize light absorption and carrier collection, such as those based on vertical nanowires (142), or by applying nanophotonics concepts to enhance the effective optical path $(109,140,141,212)$. A combination of these approaches could lead to larger PCE for ultrathin MAPI solar cells that takes full advantage of the indirect bandgap without suffering from bulk recombination.

a

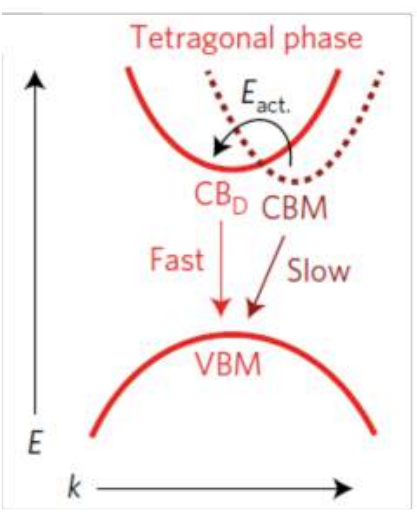

b
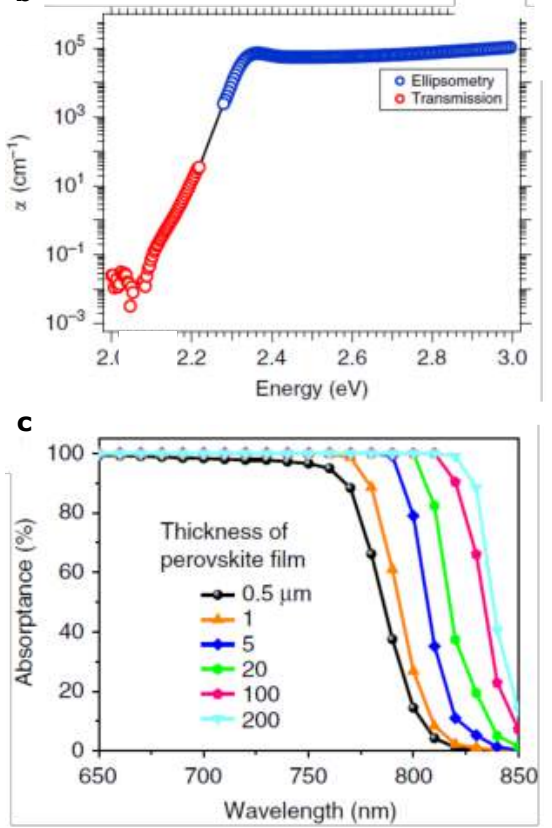

d

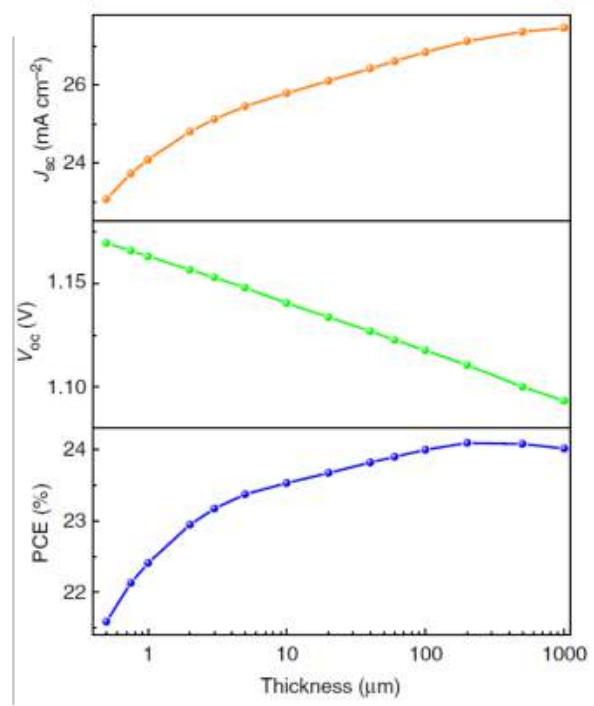

Figure 30 - Indirect-band gap and effect of crystal thickness on caracterisation techniques and device performances. a) Proposed band diagram and activation energy for second-order recombination in the MAPI thin film (from ref.(200)). b) MAPB-SC absorption coefficient obtained by the combination of transmission (red circles) and ellipsometry (blue circles) data. For transmission data $\alpha$ is calculated from Beer-Lambert law (crystal thickness 2.13 $\mathrm{mm}$ ) and for ellipsometry from the attenuation coefficient $\mathrm{k}$ (from ref.(52)) c) Calculated absorption of MAPPI films with different thickness by taking into account below band gap (indirect bandgap) absorption d) Calculated ideal dependence of $\mathrm{J}_{S} C, \mathrm{~V}_{O} C$, and PCE of the SC solar cells on the thickness of the thin SC (c and d from ref.(93)).

\section{Conclusion}

In conclusion, synthesis of MAPX SC thin films and patterned thin films for integration in solar cells remain challenging and time consuming as opposed to the fabrication of PC films. In addition, the overall material properties are dominated by relatively poor surface properties. This, in combination with the little amount of research that has been carried out in optimizing device architecture by taking into account of SC properties, have resulted so far in the record performance of SC solar cells being on the order of average PC cells. However be believe that SC perovskite remain very promising materials. First, the field is only emerging and the number of research group working on SC MAPX SC is very low (compared to PC). Yet, very interesting strategies have been developed for the synthesis of high quality bulk SC in the last year and are waiting to be implemented for thin film, or as alternative be inspired from a recent innovation in a different field ((213)). Strategies to passivate surfaces were developed with great success for PC-solar cells and could be transfer to SC, along with a rational cell design, to drastically reduce surface recombination and exploit the superior electronic properties of SC. In addition, SC present several unique features such as intrinsic higher stability, and offer the possibility to rationally exploit MAPX anisotropic properties. Control over crystallographic orientation and chemical termination in devices could in fact allow to i) reduce surface recombination and improve charge transfer by selecting a facet (and its termination) with lower defect density and better energy levels alignment ii) improve charge transport by selecting the crystal orientation with optimal transport properties with respect to the external electrode iii) tailor surface treatments and interfacial layers towards crystal orientation and termination (that can also benefit stability). A rational holistic design of the device architecture is key to take 


\section{References}

(1) T, M. et al. Journal of the American Chemical Society 2009, 131, 6050-1.

(2) Green, M. A.; Ho-baillie, A.; Snaith, H. J. Nature Photonics 2014, 8.

(3) Jena, A. K.; Kulkarni, A.; Miyasaka, T. Chemical Reviews 2019, 119, 3036-3103.

(4) N.R.E.L. Best research-cell efficiencies https : //www.nrel.gov/pv/assets/pdfs/best-researchcell-efficiencies.20200919.pdf.

(5) Dong, Q. et al. Science express 2015, 43210, 1-8.

(6) Liu, Y. et al. Science China Chemistry 2017, 60, 1367-1376.

(7) Wang, W. et al. Advanced Materials 2020.

(8) Kim, H. D. et al. Advanced Materials 2016, 28, 917-922.

(9) Reid, O. G. et al. ACS Energy Letters 2016, 1, 561-565.

(10) Yang, M. et al. Physical Chemistry Chemical Physics 2017, 19, 5043-5050.

(11) Doherty, T. A. S. et al. Nature 2020, 580.

(12) Adhyaksa, G. W. P. et al. Advanced Materials 2018, 30, 1-9.

(13) Shao, Y. et al. Energy and Environmental Science 2016, 9, 1752-1759.

(14) Yang, B. et al. Advanced Functional Materials 2017, 27, 1-8.

(15) Xing, J. et al. Physical Chemistry Chemical Physics 2016, 18, 30484-30490.

(16) Yun, J. S. et al. Advanced Energy Materials 2016, 6, 1-8.

(17) Wang, K. et al. Journal of Materials Chemistry A 2019.

(18) Wang, Q. et al. Energy and Environmental Science 2016, 0-24.

(19) Wang, Q. et al. Journal of Inorganic Materials 2016, 31, 1063-1067.

(20) Park, C. et al. Advanced Functional Materials 2017, 1703546, 1-8.

(21) Divitini, G et al. Nature Energy 2016, 1.

(22) Brunetti, B. et al. Nature Publishing Group 2016.

(23) Yin, J. et al. Journal of Physical Chemistry Letters 2015, 6, 1396-1402.

(24) Wang, T. et al. Energy and Environmental Science 2017, 10, 509-515.

(25) Murali, B. et al. ACS materials letters 2019.

(26) Turedi, B. et al. ACS Energy Letters 2021, 6.

(27) Alsalloum, A. Y. et al. ACS Energy Letters 2020, 5, 657-662.

(28) Shockley, W.; Queisser, H. J. Journal of Applied Physics 1961, 32, 510-519.

(29) Fu, Y. et al. Journal of the American Chemical Society 2015, 137, 5810-5818.

(30) Huang, J.; Shao, Y.; Dong, Q. Journal of Physical Chemistry Letters 2015, 6, 3218-3227.

(31) Haque, M. A.; Troughton, J.; Baran, D. Advanced Energy Materials 2019, 1902762, 1902762.

(32) Dang, Y. et al. CrystEngComm 2016, 18, 4476-4484.

(33) Li, S. et al. Crystals 2018, 8, 37-48.

(34) Liu, Y.; Yang, Z.; Liu, S. F. Advanced Science 2018, 5.

(35) Cheng, X. et al. Advanced Functional Materials 2019, 1905021, 1-20.

(36) Wang, X. et al. Solar RRL 2019, 3, 1800294.

(37) Zhang, Y.; Yun, J.-h.; Wang, L. Energies 2020.

(38) Li, J. et al. Advanced Functional Materials 2020, 2008684, 1-35.

(39) Arya, S. et al. Progress in Solid State Chemistry 2020, 60.

(40) Maggiora, J.; Li, F.; Zheng, R. Physica Status Solidi - Rapid Research Letters 2021, 15, 1-22.

(41) Feng, A. et al. Chemistry of Materials 2020. 
(42) Zhang, X.; Li, F.; Zheng, R. Materials Chemistry C 2020.

(43) Li, L. et al. Nano Research 2020, 12.

(44) Su, J.; Chen, D. P.; Lin, C. T. Journal of Crystal Growth 2015, 422, 75-79.

(45) Su, J. et al. Crystal Research and Technology 2016, 51, 650-655.

(46) Yang, H. et al. Journal of Materials Chemistry C 2018, 6, 11707-11713.

(47) Yao, F. et al. Nature Communications 2020, 11, 1-9.

(48) Poglitsch, A; Weber, D The Journal of Chemical Physics 1987, 6373, DOI: 10.1063/1.453467.

(49) Dang, Y. et al. CrystEngComm 2015, 17, 665-670.

(50) Lian, Z. et al. Scientific Reports 2015.

(51) Lian, Z. et al. Journal of the American Chemical Society 2016, 138, 9409-9412.

(52) Wenger, B. et al. Nature Communications 2017.

(53) Jiang, X. et al. ACS Energy Letters 2020, 1797-1803.

(54) Yang, C. et al. ACS Energy Letters 2019, 4, 2579-2584.

(55) Meggiolaro, D. et al. Energy and Environmental Science 2018, 11, 702-713.

(56) Shi, D. et al. Science 2015, 347, 519-522.

(57) Hongxia, S. et al. Journal of Materials Science 2019, 54, 11596-11603.

(58) Yang, Y. et al. Nature Communications 2015, 6, 1-6.

(59) Zhou, H. et al. RSC Advances 2015, 5, 85344-85349.

(60) Tidhar, Y. et al. Journal of the American Chemical Society 2014.

(61) Chen, F. et al. Crystal Growth and Design 2018, 3132-3137.

(62) Zhang, L. et al. Crystal Growth and Design 2018, 18, 6652-6660.

(63) Zhang, L. et al. ACS applied materials $\&$ interfaces 2020.

(64) Kadro, J. et al. Scientific Reports 2015.

(65) M, P.; Predeep, P. Materials Chemistry and Physics 2020, 239, 122084.

(66) Liu, Y. et al. Advanced Materials 2015, 27, 5176-5183.

(67) Jancik, J. et al. Crystal Growth and Design 2020, 20, 1388-1393.

(68) Gupta, R. et al. Journal of Crystal Growth 2020, 125598.

(69) Saidaminov, M. I. et al. Nature Communications 2015, 6, 1-6.

(70) Liu, Y. et al. Materials Today 2018.

(71) Maculan, G. et al. Journal of Physical Chemistry Letters 2015, 6, 3781-3786.

(72) Amari, S. et al. Crystal Growth and Design 2020, 20, 1665-1672.

(73) Huang, Y. et al. Journal of Materials Chemistry A 2017, 5, 8537-8544.

(74) Zhumekenov, A. A. et al. ACS Energy Letters 2016, 1, 32-37.

(75) Han, Q. et al. Advanced Materials 2016, 28, 2253-2258.

(76) Saidaminov, M. I. et al. Chemical Communications 2015, 51, 17658-17661.

(77) Wang, W. et al. CrystEngComm 2018, 20, 1635-1643.

(78) Taiyang, Z. et al. ChemComm 2015.

(79) Ng, M.; Halpert, J. E. RSC Advances 2020, 10, 3832-3836.

(80) Dang, Y. et al. Angewandte Chemie - International Edition 2016, 55, 3447-3450.

(81) Song, J. et al. Advanced Optical Materials 2017, 5, 1-8.

(82) Chen, J. et al. Journal of the American Chemical Society 2017, 139, 13525-13532.

(83) Zhang, M. et al. CrystEngComm 2017, 19, 6797-6803.

(84) Saidaminov, M. I. et al. Advanced Optical Materials 2017, 5.

(85) Chen, Y.-X. et al. Journal of the American Chemical Society 2016, 138, 16196-16199.

(86) Zhao, J. et al. Science Bulletin 2017, 62, 1173-1176.

(87) Nguyen, V.-C. et al. Applied Physics Letters 2016. 
(88) Liu, Y. et al. Advanced Materials 2016, 28, 9204-9209.

(89) Rao, H.-S. et al. Royal Society of Chemistry 2017, 53, 5163-5166.

(90) Rao, H.-S. et al. Advanced Materials 2017.

(91) Yang, Z. et al. Advanced Materials 2018.

(92) Yue, H.-L.; Sung, H.-H.; Chen, F.-C. Advanced Electronic Materials 2018, 4, 1-7.

(93) Chen, Z. et al. Nature Communications 2017, 8, 1-7.

(94) Di, H. et al. ACS Omega 2020, 5, 23111-23117.

(95) Kong, W. et al. Advanced Energy Materials 2020, 10, 1-8.

(96) Li, Y. et al. Nano Research 2018, 11, 3306-3312.

(97) Peng, W. et al. Advanced Materials 2016, 28, 3383-3390.

(98) Zhumekenov, A. A. et al. ACS Energy Letters 2017, 2, 1782-1788.

(99) Lei, Y. et al. Nature 2020, 583, 790-795.

(100) Li, Y.-W. et al. Chinese Physics Letters 2020, 37, 018101.

(101) Zhang, L. et al. ACS Applied Materials and Interfaces 2020, 12, 39834-39840.

(102) Zheng, J.-Y. et al. Scientific Reports 2019, 9, 1-9.

(103) Lv, Q. et al. Journal of Materials Chemistry C 2018, 6, 4464-4470.

(104) Lv, Q.; Zhang, L. ChemistrySelect 2020, 5, 14788-14791.

(105) Schlipf, J. et al. Scientif Reports 2018.

(106) Koenderink, A. F.; Polman, A. Science 2015, 348, 516-521.

(107) Wang, K. et al. Advanced Materials 2020, 2000306, 1-19.

(108) Tang, J. et al. Nature Nanotechnology 2011, 6, 568-572.

(109) Oener, S. Z. et al. Applied Physics Letters 2015, 106.

(110) Deng, W. et al. Advanced Materials 2016, 28, 2201-2208.

(111) Deng, W. et al. Advanced Materials 2020, 1908340, 1-9.

(112) Oener, S. Z. et al. Nano Letters 2017, 17, 6557-6563.

(113) Feng, J. et al. Advanced Materials 2016, 28, 3732-3741.

(114) Wu, J. et al. Chemistry of Materials 2018.

(115) Lee, L. et al. Nature Communications 2017, 8, 1-8.

(116) Fang, H.-h. et al. Materials Science 2016, 1-10.

(117) Lei, Y. et al. Advanced Materials 2018, 30, 1-8.

(118) Gu, Z. et al. Small 2017, 13, 1-7.

(119) Gu, Z. et al. Science Advances 2018.

(120) Gong, X. et al. Nature Communications 2019, 10, 1-7.

(121) Liu, J. et al. Journal of Physical Chemistry Letters 2019, 10, 97-101.

(122) Musiienko, A. et al. Energy and Environmental Science 2019, 12, 1413-1425.

(123) Mora-Seró, I. et al. Energy and Environmental Science 2009, 2, 678-686.

(124) Leever, B. J. et al. Advanced Energy Materials 2012, 2, 120-128.

(125) Duijnstee, E. A. et al. ACS Energy Letters 2020, 376-384.

(126) Wu, B. et al. Advanced Energy Materials 2016, 201600551, 1-9.

(127) Lv, Q. et al. CrystEngComm 2017, 19, 901-904.

(128) Ecker, B. R. et al. Advanced Materials Interfaces 2018, 5, 1-11.

(129) Wang, C. et al. The Journal of Physical Chemistry 2018, 3513-3522.

(130) Li, Y. L. et al. The Journal of Physical Chemistry 2017, 6-12.

(131) Murali, B. et al. ACS Energy Letters 2016.

(132) Murali, B. et al. ACS Energy Letters 2017, 2, 846-856.

(133) Rappich, J. et al. Solar RRL 2020, 4, 1900216. 
(134) Chen, Z. et al. ACS Energy Letters 2019, 4, 1258-1259.

(135) Park, J. et al. ChemSusChem 2020, 1-9.

(136) Xiao, Z. et al. Nature Materials 2015, 14, 193-197.

(137) Dong, Q. et al. Advanced Materials 2016.

(138) Liu, Y. et al. Advanced Functional Materials 2019, 29, 1-6.

(139) Song, Y. et al. Nature Communications 2020, 11, 1-8.

(140) Sciacca, B. et al. Nano Letters 2014, 14, 5891-5898.

(141) Brongersma, M.; Cui, Y.; Fan, S. Nature Materials 2014, 13.

(142) Wallentin, J. et al. Science 2013, 339, 1057-1060.

(143) Polman, A. et al. Science 2016, 352.

(144) Sherkar, T. S. et al. ACS Energy Letters 2017.

(145) Jariwala, S. et al. Joule 2019, 3, 3048-3060.

(146) Lu, H. et al. Physcial Chemistry Chemical Physics 2017.

(147) Yang, Y. et al. Nature Energy 2017, 2, 1-7.

(148) Cheng, X. et al. Journal of Materials Chemistry C 2018, 6, 1579-1586.

(149) Song, J. et al. The Journal of Physical Chemistry Letters 2020.

(150) Haruyama, J. et al. Journal of Physical Chemistry Letters 2014, 5, 2903-2909.

(151) Cho, N. et al. Nature Communications 2016, 7, 1-11.

(152) Ding, J. et al. Materials Chemistry and Physics 2018, 204, 222-227.

(153) Ding, J. et al. Journal of Physical Chemistry Letters 2018, 9, 216-221.

(154) Geng, W. et al. Journal of Materiomics 2015, 1, 213-220.

(155) Leblebici, S. Y. et al. Nature Energy 2016, 1, 1-7.

(156) Jiang, C.; Zhang, P. Journal of Applied Physics 2018, 123.

(157) Docampo, P. et al. APL Materials 2014, 2.

(158) Giesbrecht, N. et al. ACS Energy Letters 2016, 1, 150-154.

(159) Liang, Q. et al. Journal of Materials Chemistry A 2015, 4, 223-232.

(160) Wu, B. et al. Nature Communications 2019, 10.

(161) Unger, E. L. et al. Chemistry of Materials 2014, 26, 7158-7165.

(162) Murata, M. et al. Solar Energy Materials and Solar Cells 2020, 208, 110409.

(163) Zhu, W. et al. Organic Electronics 2020, 78, 105598.

(164) Kim, D. et al. Journal of Physical Chemistry C 2019, 123, 14144-14151.

(165) Ding, J. et al. ACS Applied Energy Materials 2020.

(166) Lv, Q. et al. IEEE Electron Device Letters 2018, 39, 1389-1392.

(167) Zuo, Z. et al. Journal of Physical Chemistry Letters 2017, 8, 684-689.

(168) Fassl, P. et al. ACS Applied Materials and Interfaces 2019, 11, 2490-2499.

(169) Zuo, Z. et al. Materials Research Bulletin 2018, 99, 466-470.

(170) Liu, Y. Q. et al. ACS Applied Materials and Interfaces 2020, 12, 3563-3571.

(171) Zheng, G. et al. Nature Communications 2018, 9, 1-11.

(172) Emara, J. et al. Advanced Materials 2016, 28, 553-559.

(173) Ono, L. K.; Qi, Y. Journal of Physical Chemistry Letters 2016, 7, 4764-4794.

(174) Quarti, C.; Angelis, F. D.; Beljonne, D. Chemistry of Materials 2017.

(175) Bae, S. et al. Solar Energy Materials and Solar Cells 2017, 160, 77-84.

(176) Zhou, J. et al. ACS Applied Materials and Interfaces 2018, 10, 35580-35588.

(177) Yang, S. et al. Science 2019, 365, 473-478.

(178) Duim, H. et al. Applied Physics Reviews 2019, 6.

(179) Akin, S. et al. Advanced Energy Materials 2020, 10, 1-29. 
(180) Castro-Méndez, A. F.; Hidalgo, J.; Correa-Baena, J. P. Advanced Energy Materials 2019, 9, 1-10.

(181) Ono, L. K.; Liu, S.; Qi, Y. Q. Angewandte Chemie Int. 2019.

(182) Zheng, X. et al. Nature Energy 2020, 5, 131-140.

(183) Huang, L. et al. Advanced Science 2020, 1902656.

(184) Zhang, M. et al. Nature Communications 2019, 10, 1-9.

(185) Lu, J. et al. Energy \& Environmental Science 2018.

(186) Zuo, L. et al. Journal of the American Chemical Society 2015, 137, 2674-2679.

(187) Stolterfoht, M. et al. Nature Energy 2018, 3, 847-854.

(188) Chen, Y. F.; Orgiu, E. ChemNanoMat 2019, 5, 290-299.

(189) Li, D. et al. ACS Applied Materials \& Interfaces 2020.

(190) Wolff, C. M. et al. Advanced Materials 2019, 31.

(191) Son, D.-y. et al. 2016, 1, 1-8.

(192) Leijtens, T. et al. Nature Communications 2013, 4, 1-8.

(193) Murugadoss, G. et al. Japanese Journal of Applied Physics 2015, 08.

(194) Pospisil, J. et al. Scientific Reports 2019, 9, 1-8.

(195) Zhao, Y. et al. The Journal of Physical Chemistry C 2017.

(196) Spalla, M. et al. ACS Applied Energy Materials 2020.

(197) Boldyreva, A. G. et al. ACS Applied Materials E Interfaces 2020, 12, 19161-19173.

(198) Akbulatov, A. F. et al. The Journal of Physical Chemistry Letters 2019.

(199) Mosconi, E.; Ronca, E.; De Angelis, F. Journal of Physical Chemistry Letters 2014, 5, 2619-2625.

(200) Hutter, E. M. et al. Nature Materials 2017, 16, 115-120.

(201) Huang, W. et al. ACS Photonics 2018, 5, 1583-1590.

(202) Niesner, D. et al. Physical Review Letters 2016, 117, 1-6.

(203) Niesner, D. et al. Proceedings of the National Academy of Sciences of the United States of America 2018, 115, 9509-9514.

(204) Stranks, S. D.; Plochocka, P. Nature Materials 2018, 17, 381-382.

(205) Azarhoosh, P. et al. APL Materials 2016, 4.

(206) Kepenekian, M. et al. ACS nano 2015.

(207) Brivio, F.; Butler, K. T.; Walsh, A. Physical Review B 2014, 155204, 1-6.

(208) Thibaud, E.; Mosconi, E.; Angelis, F. D. The journal of Physical Chemistry Letters 2016.

(209) Zheng, F. et al. Nano letters 2015.

(210) Singh, R. K. et al. Materials Today: Proceedings 2017, 4, 12661-12665.

(211) Xiao, Y. Y. et al. Sustainable Energy and Fuels 2020, 4, 824-831.

(212) Polman, A.; Atwater, H. A. Nature Materials 2012, 11, 174-177.

(213) Sciacca, B. et al. Advanced Materials 2016, 28, 905-909. 